Universidade de São Paulo

Instituto de Astronomia, Geofísica e Ciências Atmosféricas

Departamento de Astronomia

Vinicius Consolini Busti

Quantificando as Inomogeneidades da

Matéria com Supernovas e Gamma-Ray

Bursts

São Paulo

2009 

Vinicius Consolini Busti

\section{Quantificando as Inomogeneidades da \\ Matéria com Supernovas e Gamma-Ray}

\section{Bursts}

Dissertação apresentada ao Departamento de Astronomia do Instituto de Astronomia, Geofísica e Ciências Atmosféricas da Universidade de São Paulo como parte dos requisitos para a obtenção do título de Mestre em Ciências.

Área de Concentração: Astronomia Orientador: Prof. Dr. José Ademir Sales de Lima

São Paulo 

À minha família. 



\section{Agradecimentos}

Ao meu orientador José Ademir Sales de Lima, por todo o apoio obtido desde a Iniciação Científica e pela escolha do tema.

À Rose Clívia Santos, pela amizade e colaboração em dois trabalhos.

A todos da minha família. Principalmente aos meus pais Francisco Aparecido Busti e Magda de Jesus Consolini Busti, à minha irmã Vanessa Aparecida Busti, à minha sobrinha Alessa Moraes e à minha namorada Angela Ramonne Pereira de Souza, por tudo.

A todos meus amigos, pelos bons momentos que passamos.

Aos professores do Departamento de Astronomia do IAG, principalmente aos professores Ruth Gruenwald, Antônio Mário Magalhães, Roberto Dias da Costa, Jorge Horvath, Sílvia Rossi, Ronaldo Eustáquio de Souza, Gastão Lima Neto e Gary Steigman pelas disciplinas lecionadas.

Ao grupo de cosmologia: José Fernando de Jesus, Rodrigo Fernandes Holanda, Felipe Andrade Oliveira, Antonio Guimarães, João Maria da Silva, João Vital Cunha Júnior, Lúcio Marassi de Souza Almeida, Maria Assunta Nobre, Rose Clívia Santos e Saulo Pereira, por todas as discussões científicas e pela amizade.

Aos membros da Representação Discente, especialmente ao Oscar Cavichia de Moraes e ao Vinicius Moris Placco pelo desenvolvimento da classe IAGTESE.

Aos funcionários do IAG, por toda a assistência prestada.

À FAPESP, pelo apoio financeiro, sob o projeto $n^{o}:$ 2006/05298-4.

Ao IAG, por propiciar um ótimo ambiente de trabalho e pesquisa.

Esta dissertação foi escrita em IATEX com a classe IAGTESE, para teses e dissertações do IAG. 

"O eterno mistério do mundo é a possibilidade de o compreendermos ... O fato de que ele seja compreensivel é um milagre."

Albert Einstein 



\section{Resumo}

Nesta dissertação estudamos como os efeitos das inomogeneidades da matéria (escura e bariônica) modificam as distâncias e afetam a determinação dos parâmetros cosmológicos. As inomogeneidades são fenomenologicamente descritas pelo parâmetro de aglomeramento $\alpha$ e quantificadas pela equação da distância proposta por Zeldovich-Kantowski-DyerRoeder (ZKDR). Além disso, utilizando amostras de Supernovas e Gamma-Ray Bursts, aplicamos um teste $\chi^{2}$ para vincular os parâmetros de dois modelos cosmológicos distintos, a saber: o modelo $\Lambda$ CDM plano e o modelo com criação de matéria escura fria.

Para o modelo $\Lambda$ CDM plano, vinculamos os parâmetros $\alpha$ e $\Omega_{M}$ considerando um prior gaussiano para a constante de Hubble $H_{0}$. Realizamos também uma análise detalhada envolvendo duas calibrações distintas associadas aos dados de Gamma-Ray Bursts: uma calibração para o modelo $\Lambda$ CDM plano e outra para o modelo cardassiano. Verificamos que os resultados são fracamente dependentes da calibração adotada.

Uma análise conjunta envolvendo Supernovas e Gamma-Ray Bursts permitiu quebrar a degenerescência entre o parâmetro de aglomeramento $\alpha$ e o parâmetro de densidade da matéria $\Omega_{M}$. Considerando a calibração dos Gamma-Ray Bursts para o modelo $\Lambda$ CDM plano, o melhor ajuste obtido foi $\alpha=1.0$ e $\Omega_{M}=0.30$, com os parâmetros restritos ao intervalos $0.78 \leq \alpha \leq 1.0$ e $0.26 \leq \Omega_{M} \leq 0.36(2 \sigma)$.

Para o modelo com criação de matéria escura consideramos também um prior gaussiano para $H_{0}$ e as amostras de Supernovas e Gamma-Ray Bursts (calibrados para o modelo $\Lambda$ CDM plano). A degenerescência entre o parâmetro $\alpha$ e o parâmetro de criação $\gamma$ foi novamente quebrada atráves de uma análise conjunta das 2 amostras de dados. Para o melhor ajuste obtivemos $\alpha=1.0$ e $\gamma=0.61$, com os parâmetros restritos aos intervalos $0.85 \leq \alpha \leq 1.0$ e $0.56 \leq \gamma \leq 0.66(2 \sigma)$. 



\section{Abstract}

In this dissertation we study how the effects of matter (baryonic and dark) inhomogeneities modify the distances thereby affecting the determination of cosmological parameters. The inhomogeneities are phenomenologically described by the clumpiness parameter $\alpha$ and quantified through the equation distance proposed by Zeldovich-Kantowski-DyerRoeder (ZKDR). Further, by using Supernovae and Gamma-Ray Bursts separately, a $\chi^{2}$ analysis was performed to constrain the parameter space for two distinct cosmological models, namely: the flat $\Lambda$ CDM model and the cold dark matter creation model.

For the flat $\Lambda \mathrm{CDM}$ model we have constrained the parameters $\alpha$ and $\Omega_{M}$ by considering a Gaussian prior for the Hubble parameter $H_{0}$. A detailed analysis was also performed involving two different calibrations associated to the Gamma-Ray Bursts data: a calibration for the flat $\Lambda$ CDM model as well as for the cardassian model. We have verified that the results are weakly dependent on the adopted calibration.

A joint analysis involving Supernovae and Gamma-Ray Bursts allowed us to break the degenerescence between the clumpiness parameter $\alpha$ and the matter density parameter $\Omega_{M}$. By considering the calibration for the flat $\Lambda C D M$ model, the best fits obtained were equal to $\alpha=1.0$ and $\Omega_{M}=0.30$ with the parameters restricted on the intervals $0.78 \leq \alpha \leq 1.0$ and $0.26 \leq \Omega_{M} \leq 0.36(2 \sigma)$.

For the dark matter creation model we have also adopted a Gaussian prior for $H_{0}$ and the Supernovae and Gamma-Ray Bursts (calibrated for the flat $\Lambda$ CDM model) samples. The degenerescence between the clumpiness parameter $\alpha$ and the creation parameter $\gamma$ was again broken trough a joint analysis of the two data sample. For the best fits we have obtained $\alpha=1.0$ and $\gamma=0.61$ with the parameters restricted on the intervals $0.85 \leq \alpha \leq 1.0$ and $0.56 \leq \gamma \leq 0.66(2 \sigma)$. 



\section{Notação e Convenções}

- Assinatura da métrica: $(+,-,-,-)$

- Índices gregos variam de 0 a 3 , índices latinos variam de 1 a 3 . Índices repetidos obedecem à convenção de Einstein.

- Derivada parcial: $\frac{\partial \phi}{\partial x^{\alpha}} \equiv \phi_{, \alpha}$

- Derivada covariante: $A_{; \beta}^{\alpha}=A_{, \beta}^{\alpha}+\Gamma_{\lambda \beta}^{\alpha} A^{\lambda}$

- Usaremos um sistema de unidades onde $c=1$, salvo menção em contrário a fim de explicitar algo em questão.

- Expressões em outro idioma serão escritas em itálico. 



\section{Informação Eletrônica}

A maioria das referências bibliográficas utilizadas nessa dissertação podem ser encontradas nas seguintes páginas da WEB:

- http://www.periodicos.capes.gov.br/

- http://adsabs.harvard.eduabstract_service.html

- http://xxx.lanl.gov/ 



\section{Lista de Figuras}

1.1 Fator de escala em função do tempo para um universo plano, $k=0$, universo fechado, $k=1$, e universo hiperbólico, $k=-1$. Em todos os casos consideramos um universo dominado somente por matéria. . . . . . . . . . 41

2.1 Determinação da distância entre o Sol e uma estrela pelo método da paralaxe trigonométrica. . . . . . . . . . . . . . . . . . 44

2.2 Modelo da bola de fogo (fireball model). . . . . . . . . . . . . . . 52

3.1 Evidência da aceleração do universo obtida pelo Supernova Cosmology Project

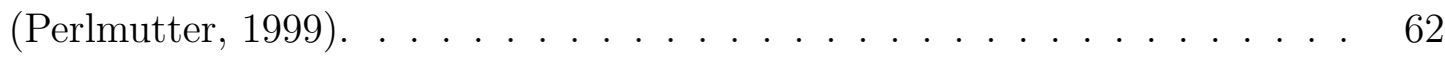

3.2 Evidência da aceleração do universo obtida pelo High-z Supernova Search (Riess et al., 1998). . . . . . . . . . . . . . . . 63

3.3 Genealogia da energia escura. . . . . . . . . . . . . . . . 64

3.4 Intervalo de modelos cosmológicos não-planos consistentes com o WMAP. . 65

3.5 Vínculos para $w_{0}$ e $w_{a}$ obtidos por Kowalski et al. (2008). . . . . . . . . . 68

4.1 Representação da propagação de um feixe de raios de luz. . . . . . . . . . . 76

4.2 Efeitos do parâmetro de expansão $\theta$ e do tensor de cisalhamento $\sigma_{a b}$ na área transversal de um feixe de luz. . . . . . . . . . . . . . . . . . . . . . . . 79

4.3 Distância de luminosidade em unidades de $c / H_{0}$ em função do redshift para vários valores do parâmetro de aglomeramento $\alpha$, com $\Omega_{M}=0.27$. . . . . 84

4.4 Distância de luminosidade em unidades de $c / H_{0}$ em função do redshift para vários valores do parâmetro de aglomeramento $\alpha, \operatorname{com} \gamma=0.6 \ldots . . . \quad 86$ 
5.1 Magnitude residual comparada a um universo vazio para supernovas e gammaray bursts. . . . . . . . . . . . . . . . . . . . . . . . . . 89

5.2 Vínculos obtidos para $\alpha$ e $\Omega_{M}$ com 307 supernovas da amostra de Kowalski et al. (2008) (Union Compilation Data) considerando um prior gaussiano em $H_{0} \ldots \ldots \ldots \ldots \ldots \ldots \ldots \ldots$

5.3 Vínculos obtidos para $\alpha$ e $\Omega_{M}$ com 69 GRBs calibrados por Schaefer (2007) considerando um prior gaussiano em $H_{0} \ldots \ldots$. . . . . . . . . . . .

5.4 Vínculos obtidos para $\alpha$ e $\Omega_{M}$ com 69 GRBs calibrados para o modelo cardassiano (Mosquera Cuesta et al., 2008) considerando um prior gaussiano em $H_{0} \ldots \ldots \ldots \ldots \ldots \ldots \ldots \ldots \ldots$

5.5 Vínculos obtidos para $\alpha$ e $\Omega_{M}$ com 307 supernovas da amostra de Kowalski et al. (2008) (Union Compilation Data) e 69 GRBs calibrados por Schaefer (2007) considerando um prior gaussiano em $H_{0}$. . . . . . . . . . . . . . .

5.6 Vínculos obtidos para $\alpha$ e $\Omega_{M}$ com 307 supernovas da amostra de Kowalski et al. (2008) (Union Compilation Data) e 69 GRBs calibrados para o modelo cardassiano (Mosquera Cuesta et al., 2008) considerando um prior gaussiano em $H_{0} \ldots \ldots \ldots \ldots \ldots \ldots \ldots \ldots \ldots \ldots \ldots$

6.1 Magnitude residual comparada a um universo vazio para supernovas e gamma-

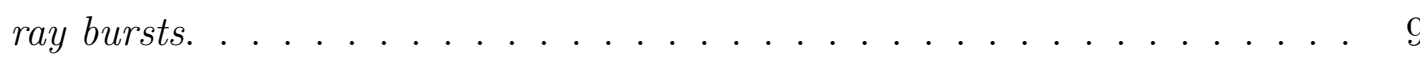

6.2 Vínculos obtidos para $\alpha$ e $\gamma$ com 307 supernovas da amostra de Kowalski et al. (2008) (Union Compilation Data) considerando um prior gaussiano em $H_{0} \ldots \ldots \ldots \ldots \ldots \ldots \ldots \ldots \ldots \ldots \ldots$

6.3 Vínculos obtidos para $\alpha$ e $\gamma$ com 69 GRBs calibrados por Schaefer (2007) considerando um prior gaussiano em $H_{0} \ldots \ldots$. . . . . . . . . . . . . . . . . 99

6.4 Vínculos obtidos para $\alpha$ e $\gamma$ com 307 supernovas da amostra de Kowalski et al. (2008) (Union Compilation Data) e 69 GRBs calibrados por Schaefer (2007) considerando um prior gaussiano em $H_{0}$. . . . . . . . . . . . . . . . 100

B.1 Vínculos obtidos para $\alpha$ e $\Omega_{M}$ com 307 supernovas da amostra de Kowalski et al. (2008) (Union Compilation Data) considerando $H_{0}$ fixo. 
B.2 Vínculos obtidos para $\alpha$ e $\Omega_{M}$ com 69 GRBs calibrados por Schaefer (2007) considerando $H_{0}$ fixo. . . . . . . . . . . . . . . . . . . . . . . . . . . . . 125

B.3 Vínculos obtidos para $\alpha$ e $\Omega_{M}$ com 69 GRBs calibrados para o modelo cardassiano (Mosquera Cuesta et al., 2008) considerando $H_{0}$ fixo. . . . . . 125

B.4 Vínculos obtidos para $\alpha$ e $\Omega_{M}$ com 307 supernovas da amostra de Kowalski et al. (2008) (Union Compilation Data) e 69 GRBs calibrados por Schaefer (2007) considerando $H_{0}$ fixo. . . . . . . . . . . . . . . . . . .

B.5 Vínculos obtidos para $\alpha$ e $\Omega_{M}$ com 307 supernovas da amostra de Kowalski et al. (2008) (Union Compilation Data) e 69 GRBs calibrados para o modelo cardassiano (Mosquera Cuesta et al., 2008) considerando $H_{0}$ fixo. . . . . . .

B.6 Vínculos obtidos para $\alpha$ e $\Omega_{M}$ com 307 supernovas da amostra de Kowalski et al. (2008) (Union Compilation Data) considerando um prior flat em $H_{0}$.

B.7 Vínculos obtidos por Santos et al. (2008) para $\alpha$ e $\Omega_{M}$ com 182 supernovas da amostra de Riess et al. (2007) considerando um prior flat em $H_{0}$. . . .

B.8 Vínculos obtidos para $\alpha$ e $\Omega_{M}$ com 69 GRBs calibrados por Schaefer (2007) considerando um prior flat em $H_{0} \ldots \ldots \ldots \ldots$. . . . . . . .

B.9 Vínculos obtidos para $\alpha$ e $\Omega_{M}$ com 69 GRBs calibrados para o modelo cardassiano (Mosquera Cuesta et al., 2008) considerando um prior flat em

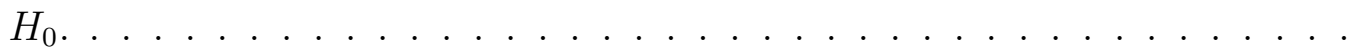

B.10 Vínculos obtidos para $\alpha$ e $\Omega_{M}$ com 307 supernovas da amostra de Kowalski et al. (2008) (Union Compilation Data) e 69 GRBs calibrados por Schaefer (2007) considerando um prior flat em $H_{0}$

B.11 Vínculos obtidos para $\alpha$ e $\Omega_{M}$ com 307 supernovas da amostra de Kowalski et al. (2008) (Union Compilation Data) e 69 GRBs calibrados para o modelo cardassiano (Mosquera Cuesta et al., 2008) considerando um prior flat em

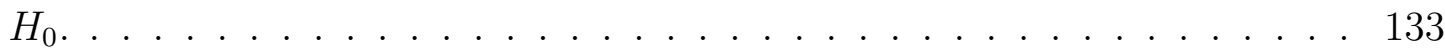





\section{Lista de Tabelas}

2.1 Resultados da calibração de Schaefer (2007). . . . . . . . . . . . . . . 56

2.2 Resultados da calibração para o modelo cardassiano. . . . . . . . . . . . . 58

5.1 Vínculos para os parâmetros $\alpha$ e $\Omega_{M}$ considerando um prior gaussiano em

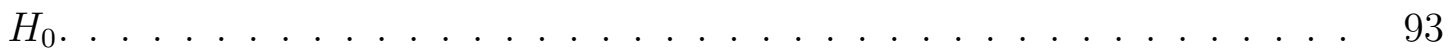

6.1 Vínculos para os parâmetros $\alpha$ e $\gamma$ considerando um prior gaussiano em $H_{0} .100$

B.1 Vínculos para os parâmetros $\alpha$ e $\Omega_{M}$ considerando $H_{0}$ fixo. . . . . . . . . . 128

B.2 Vínculos para os parâmetros $\alpha$ e $\Omega_{M}$ considerando um prior flat em $H_{0}$. . 133 



\section{Sumário}

1. Modelo Padrão da Cosmologia . . . . . . . . . . . . . . . . . . . . . . . 33

1.1 Introdução . . . . . . . . . . . . . . . . . . . . . . . . 33

1.2 A Teoria da Relatividade Geral (TRG) . . . . . . . . . . . . . . . . 34

1.3 Métrica FLRW (Friedmann-Lemaître-Robertson-Walker) . . . . . . . . . . 35

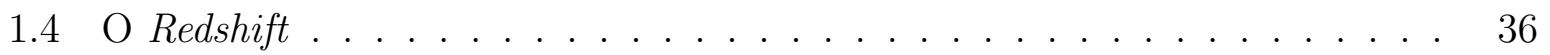

1.5 Tensor de Energia-Momento . . . . . . . . . . . . . . . . . 37

1.6 Dinâmica Cosmológica . . . . . . . . . . . . . . . . . . . . . . . . . . 38

2. A Escada de Distância Cósmica . . . . . . . . . . . . . . . . . . . . . . . . 43

2.1 Introdução . . . . . . . . . . . . . . . . . . . . . . . . . 43

2.2 Indicadores Primários de Distância . . . . . . . . . . . . . . . . 43

2.2.1 Paralaxe Trigonométrica . . . . . . . . . . . . . . . . . 44

2.2.2 Movimento Próprio . . . . . . . . . . . . . . . . . . . . . 45

2.2.3 Luminosidade Aparente . . . . . . . . . . . . . . . . . 45

2.3 Indicadores Secundários de Distância . . . . . . . . . . . . . . . . . . 47

2.3.1 A Relação de Tully-Fisher . . . . . . . . . . . . . . . . . . . . . . . 47

2.3.2 A Relação de Faber-Jackson . . . . . . . . . . . . . . . . . . . . . 48

2.3.3 O Plano Fundamental . . . . . . . . . . . . . . . . . . . . 48

2.3.4 Supernovas Tipo Ia . . . . . . . . . . . . . . . . . . . . . . . . . 48

2.4 Medidas de Distância em Espaço-Tempo Curvo . . . . . . . . . . . . . . . 49

2.4.1 Distância de Luminosidade . . . . . . . . . . . . . . . . . . . . . 49

2.4 .2 Distância de Diâmetro Angular . . . . . . . . . . . . . . 50 
2.5 Gamma-Ray Bursts ............................ 51

2.5.1 Método de Calibração de Schaefer . . . . . . . . . . . . . . . . . . 52

2.5.2 Calibração para o Modelo Cardassiano . . . . . . . . . . . . 57

2.5.3 Calibração utilizando Supernovas Ia . . . . . . . . . . . . . . . 58

3. Cosmologias Aceleradas . . . . . . . . . . . . . . . . . . . 61

3.1 Introdução . . . . . . . . . . . . . . . . . . . . . . . . . 61

3.2 A Constante Cosmológica $\Lambda \ldots \ldots$. . . . . . . . . . . . 63

3.3 Matéria X . . . . . . . . . . . . . . . . . 66

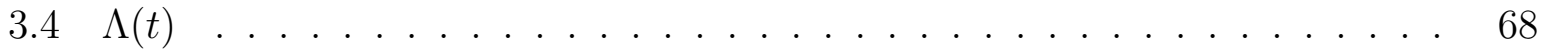

3.5 Gás de Chaplygin . . . . . . . . . . . . . . . . . . . . . . . 70

3.6 Modelo Cardassiano . . . . . . . . . . . . . . . . . . . . . . . . . 71

3.7 Modelo com Criação de Matéria . . . . . . . . . . . . . . . . . . 72

4. Influência das Inomogeneidades na Propagação da Luz: O Método de Dyer-Roeder 75 4.1 Introdução . . . . . . . . . . . . . . . . . . . . . . . . . . 75

4.2 A Equação Óptica de Sachs . . . . . . . . . . . . . . . . . . . 75

4.3 Equação ZKDR para o modelo $\Lambda$ CDM . . . . . . . . . . . . . . . . 80

4.4 Equação ZKDR para o modelo com criação de matéria . . . . . . . . . . . 84

5. Vínculos Observacionais para $\alpha$ e $\Omega_{M} \ldots \ldots \ldots \ldots$. . . . . . . . 87

5.1 Introdução . . . . . . . . . . . . . . . . . . 87

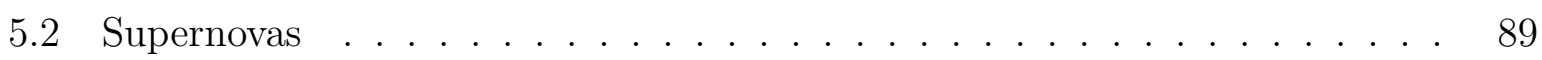

5.3 Gamma-Ray Bursts . . . . . . . . . . . . . . . . . . . . . . . 89

5.4 Supernovas e Gamma-Ray Bursts . . . . . . . . . . . . . . . . . . . . 91

6. Vínculos Observacionais para $\alpha$ e $\gamma \ldots \ldots \ldots$. . . . . . . . . . . 95

6.1 Introdução . . . . . . . . . . . . . . . . . . . . . 95

6.2 Supernovas . . . . . . . . . . . . . . . . . . . . . . . . 98

6.3 Gamma-Ray Bursts . . . . . . . . . . . . . . . . . . . . . . 98

6.4 Supernovas e Gamma-Ray Bursts . . . . . . . . . . . . . . . . . . . . . . . 99

7. Conclusões e Perspectivas . . . . . . . . . . . . . . . . . . . 101 
A. Equação do Desvio Geodésico

B. Influência dos Priors sobre $H_{0}$ nos Vínculos Cosmológicos . . . . . . . . . . . . 123

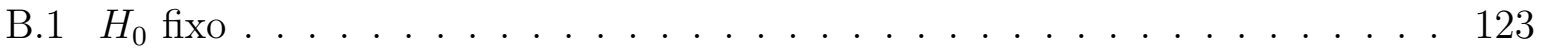

B.1.1 Supernovas . . . . . . . . . . . . . . . . . . 123

B.1.2 Gamma-Ray Bursts . . . . . . . . . . . . . . . . . . . 124

B.1.3 Supernovas e Gamma-Ray Bursts . . . . . . . . . . . . . . . . . . 126

B.2 Prior flat em $H_{0} \quad \ldots \ldots \ldots \ldots$

B.2.1 Supernovas . . . . . . . . . . . . . . . . . . . 128

B.2.2 Gamma-Ray Bursts . . . . . . . . . . . . . . . . . . . . 129

B.2.3 Supernovas e Gamma-Ray Bursts . . . . . . . . . . . . . . . . . 131 



\section{Introdução}

A cosmologia é o ramo da física que estuda o universo como um todo, sua origem, evolução e composição. A cosmologia moderna se iniciou com o modelo proposto por Einstein (1917), utilizando sua então recém-formulada teoria da Relatividade Geral (TRG). A TRG é uma teoria sobre o espaço, tempo e gravitação. Atualmente, é considerada o melhor arcabouço teórico para investigar as propriedades do universo em larga escala. Einstein também propôs o Princípio Cosmológico, o fato de que não existem observadores privilegiados, o que implica que o universo em grande escala deve ser homogêneo e isotrópico. $\mathrm{Na}$ época, acreditava-se que o universo era estático, fato que fez com que Einstein adicionasse a sua teoria a constante $\Lambda$, conhecida como constante cosmológica, e que pode ter um papel fundamental na evolução do universo. Assim, o modelo de Einstein era composto por matéria homogeneamente distribuída, cuja ação gravitacional era contrabalançada pela constante cosmológica gerando um universo estático. No mesmo ano, de Sitter (1917) obteve soluções para universos estáticos e em expansão constituídos apenas de uma constante cosmológica.

Posteriormente, Alexander Friedmann percebeu que as equações de Einstein para universos homogêneos e isotrópicos com matéria permitiam soluções dinâmicas, com $\Lambda=0$ e $\Lambda \neq 0$. Foram obtidas soluções para um universo com curvatura positiva (Friedmann, 1922) e com curvatura negativa (Friedmann, 1924). Lemaître (1927) também analisou a possibilidade de um universo em expansão, discutindo sobre uma possível velocidade de recessão de nebulosas extragalácticas.

No final da década de 20 as especulações de um universo em expansão receberam suporte observacional. Hubble (1929) descobriu que quanto maior a distância de uma galáxia maior era a sua velocidade de recessão, uma lei empírica atualmente conhecida 
como lei de Hubble. Dentro da TRG, a lei de Hubble pode ser entendida como a expansão do próprio espaço entre as galáxias. Einstein, quando ciente das observações astronômicas, chegou a dizer que a introdução da constante cosmológica foi o maior erro da sua vida. Juntamente com de Sitter, Eintein estudou um modelo onde a constante cosmológica é nula e a geometria da seção espacial é euclidiana, conhecido atualmente como modelo de Einstein-de Sitter (Einstein e de Sitter, 1932). Já Lemaitre (1934) propôs a idéia de um átomo primordial, uma vez que se o universo está expandindo houve um momento em que toda matéria estaria concentrada em um único ponto. O instante inicial foi denominado por Fred Hoyle de Big Bang. Tais soluções expansionistas foram classificadas posteriormente de uma forma geral por Robertson (1936) e Walker (1936), sendo atualmente chamada em sua forma geral de métrica de Friedmann-Lemaître-Robertson-Walker (FLRW).

Como o universo passou por um estágio denso e quente, essas condições eram propícias para a formação dos elementos químicos, sendo essa questão estudada inicialmente por Alpher et al. (1948). Somente na década de 60 é que realmente foi demonstrada a origem dos elementos leves como D, ${ }^{3} \mathrm{He},{ }^{4} \mathrm{He}$ e Li, através da medição de abundâncias relativas, tendo esses elementos químicos sido produzidos segundos após o Big Bang. A teoria de formação de elementos químicos no início do universo é chamada de nucleossíntese primordial. Gamow também propôs a existência de uma radiação de fundo (Alpher et al., 1948) que permeia o universo, visto que inicialmente matéria e radiação interagiam e, devido à expansão, houve um momento onde a interação cessou e a radiação passou a se propagar livremente. Tal radiação, conhecida modernamente como radiação cósmica de fundo, foi medida por Penzias e Wilson (1965), o que fortaleceu ainda mais o modelo expansionista.

Os três observáveis listados acima: expansão do universo, abundâncias relativas de elementos químicos leves e a radiação cósmica de fundo, juntamente com a TRG constituem a base do modelo de universo quente (Hot Big Bang), onde tais observações são confirmadas com erros da ordem de poucos pontos percentuais presentemente. Esse modelo, com a hipótese de um universo dominado por matéria e $\Lambda=0$, constituiu o modelo cosmológico padrão até quase o fim da década de 90. O cenário mudou em 1998 quando dois grupos independentes, o Supernova Cosmology Project e o High-z Supernova Search, afirmaram que a expansão do universo é acelerada (Riess et al., 1998; Perlmutter, 1999). Eles obtiveram esse resultado ao tratar as supernovas do tipo Ia como velas padrão, através da identi- 
ficação de uma correlação empírica entre a altura do pico da curva de luz e o tempo de decaimento. Dentro da TRG, tal resultado sugere que o universo é atualmente dominado por um fluido exótico que possui pressão negativa, chamado de energia escura.

Evidências para a existência de energia escura também são encontradas a partir de análises independentes provenientes de vários ramos da cosmologia. O estudo das anisotropias no espectro de potência da radiação cósmica de fundo (Komatsu et al., 2008), de estruturas em grande escala (Cole et al., 2005; Tegmark et al., 2004), observações em raios X de aglomerados de galáxias (Allen et al., 2002; Lima et al., 2003; Allen et al., 2008), objetos velhos em altos redshifts (Krauss, 1997; Alcaniz e Lima, 1999b, 2001; Alcaniz et al., 2003; Cunha e Santos, 2004; Cunha et al., 2007; Lima et al., 2009), tamanho angular de fontes de rádio compactas (Gurvits et al., 1999; Lima e Alcaniz, 2002) e rádio-galáxias (Daly e Guerra, 2002), gamma-ray bursts (Schaefer, 2007; Mosquera Cuesta et al., 2008) e outros, são testes complementares que mostram a existência dessa componente.

Determinar a natureza da energia escura é o maior desafio da cosmologia moderna. O candidato mais simples à energia escura é a constante cosmológica, que pode ser associada à energia do vácuo dos campos quânticos. Entretanto, existem vários candidatos além da constante cosmológica, dentre os quais podemos citar: campo escalar (Ratra e Peebles, 1988; Maia e Lima, 2002; Carvalho et al., 2006, 2008), matéria X (Turner e White, 1997), decaimento do vácuo (Bronstein, 1933; Ozer e Taha, 1986, 1987), gás de Chaplygin (Kamenshchik et al., 2001; Bilić et al., 2002; Bento et al., 2002). Atualmente, todos esses candidatos ajustam bem os dados observacionais (Lima, 2004; Copeland et al., 2006; Cunha, 2006).

Uma outra forma de encarar o problema seria achar um mecanismo que produzisse aceleração sem a necessidade da inclusão da energia escura. Modelos com criação de matéria foram propostos (Lima et al., 1996, 2008), onde a criação de partículas geraria uma pressão negativa responsável pela aceleração. Também é possível gerar aceleração modificando-se a TRG tomando, ao invés do escalar de curvatura de Ricci $R$ para a obtenção das equações de Einstein, funções de $R$, que são teorias conhecidas como $f(R)$ (Capozziello et al., 2003; Carroll et al., 2004). Outra possibilidade é considerar que vivemos em um universo com dimensões extras, mas apenas a gravidade as acessa, sendo possível uma aceleração do universo em seu estágio atual. Teorias com dimensões extras são geralmente inspiradas em 
teorias de cordas ou membranas (Randall e Sundrum, 1999a,b; Arkani-Hamed et al., 1999; Pires et al., 2006).

Pelo Princípio Cosmológico, temos que o universo deve ser homogêneo e isotrópico apenas em grandes escalas. Mas a luz sente as inomogeneidades locais, tendo sua trajetória desviada da prevista por um universo homogêneo e isotrópico em todas as escalas. Esse efeito de magnificação, ou demagnificação, do feixe de luz altera as distâncias. Os primeiros estudos sobre este efeito foram efetuados por Zeldovich (1964) e Kantowski (1969). Dyer e Roeder (1972) estudaram como a distância de diâmetro angular é afetada quando consideramos que a trajetória do fóton está contida em uma região sem a presença de matéria. A distância é obtida através da equação óptica de Sachs (1961), que dá a evolução da área transversal de um feixe de luz ao longo de sua trajetória no espaço-tempo. Em um trabalho posterior, Dyer e Roeder (1974) analisaram o caso em que a trajetória do fóton está contida em uma região em que apenas parte da matéria é distribuída de forma homogênea, sendo essa parte quantificada pelo parâmetro de aglomeramento $\alpha$. Devido ao processo de formação de estruturas, esperamos que haja uma dependência do parâmetro de aglomeramento com o redshift, assim como uma dependência com a direção ao longo da linha de visada.

Diante dessa conjuntura, essa tese objetiva vincular os parâmetros cosmológicos de alguns modelos, assim como a influência do parâmetro de aglomeramento. O formato da dissertação é descrito a seguir.

No capítulo 1, fazemos uma rápida revisão de aspectos importantes do modelo padrão da cosmologia. Inicialmente discutimos os fundamentos básicos da relatividade geral e como o Princípio Cosmológico leva à métrica FLRW através de argumentos de simetria. Em seguida, apresentamos as equações que governam a evolução dos componentes do universo, conhecidas como equações de Friedmann.

No capítulo 2, estudamos a escada de distância cósmica, através dos indicadores primários e secundários de distância. Também vemos como as distâncias são definidas quando levamos em conta a expansão do universo. De grande relevância para a presente dissertação é o método baseado na utilização de gamma-ray bursts como velas padrão, que vemos no final do capítulo.

No capítulo 3, estudamos modelos onde o estágio atual do universo é acelerado. Trata- 
mos de alguns modelos onde um fluido exótico, conhecido como energia escura, é adicionado às equações de Einstein. Modelos em que tal fluido não é necessário, como o modelo cardassiano e o modelo com criação de matéria, são também considerados.

No capítulo 4, estudamos os efeitos das inomogeneidades. Primeiramente, discutimos a equação óptica de Sachs e, a partir desta, deduzimos a equação de Zeldovich-KantowskiDyer-Roeder (ZKDR) para os modelos de interesse: $\Lambda$ CDM e criação de matéria.

No capítulo 5, apresentamos os resultados dos vínculos obtidos pela análise estatística através da utilização dos dados de supernova Ia e gamma-ray bursts ao modelo $\Lambda$ CDM.

No capítulo 6, apresentamos os resultados para os vínculos obtidos ao modelo com criação de matéria, enquanto no capítulo 7 apresentamos as considerações finais e algumas perspectivas de nosso trabalho.

Finalmente, com o intuito de facilitar a consulta de estudantes e pesquisadores interessados nesta área de pesquisa, informamos que os resultados originais dessa dissertação estão apresentados nos capítulos 5 e 6 e no apêndice B. 
Capítulo 1

\section{Modelo Padrão da Cosmologia}

\subsection{Introdução}

Atualmente, o modelo que melhor explica o universo é o cenário do Big Bang, considerado o modelo padrão da cosmologia. Tal modelo tem como base quatro pilares, sendo um teórico e três observacionais. Teoricamente, ele se baseia na teoria da Relatividade Geral (TRG), que até o presente momento explica todos os fenômenos gravitacionais observados com grande precisão, onde os primeiros modelos cosmológicos foram propostos por Einstein (1917), de Sitter (1917), Friedmann (1922, 1924), Lemaître (1927) e Einstein e de Sitter (1932). O primeiro pilar observacional é a expansão do universo descoberta por Hubble (1929), através da observação de que quanto maior a distância de uma galáxia, maior é sua velocidade de afastamento em relação à Via Láctea. Outra observação astronômica importante está relacionada à abundância dos elementos leves, como o hélio, deutério e o lítio, formados nos instantes iniciais do universo após o Big Bang. Alpher et al. (1948) previram estas abundâncias, mas as observações somente começaram a partir da década de 60. Por fim, Gamow previu a existência de uma radiação de fundo que permeia o universo (Alpher et al., 1948), uma vez que no passado matéria e radiação interagiam, mas devido à expansão do universo, a diluição destas fez com que a interação cessasse e a radiação passasse a se propagar livremente, conhecida hoje como radiação cósmica de fundo. Ela foi medida pela primeira vez por Penzias e Wilson (1965).

Neste capítulo, discutimos alguns aspectos desse modelo que são relevantes para a dissertação. Iniciando por sua base teórica, a Relatividade Geral. O conteúdo aqui apresentado faz parte dos livros texto de cosmologia e relatividade geral, como por exemplo Weinberg (1972) e Peebles (1993). 


\subsection{A Teoria da Relatividade Geral (TRG)}

A TRG é uma teoria de espaço, tempo e gravitação, proposta por Einstein em 1915 para incorporar os efeitos gravitacionais à relatividade especial. Esta teoria explicava os fenômenos mecânicos e eletromagnéticos mas não levava o campo gravitacional em consideração. Muito mais do que uma simples generalização, a TRG mudou nossa forma de compreender o espaço-tempo.

A TRG se baseia em dois conjuntos de idéias. O primeiro se trata do princípio de equivalência, o fato de que todos os corpos caem da mesma forma em um campo gravitacional, independentemente de sua natureza. Assim sendo, o movimento de partículas em queda livre define um conjunto preferencial de curvas no espaço-tempo, assim como na relatividade especial, o que sugere que podemos atribuir propriedades do campo gravitacional à própria estrutura do espaço-tempo. Logo, a gravidade não age como um campo, mas sim deforma a geometria do espaço-tempo.

O segundo conjunto de idéias está relacionado ao princípio de Mach. Este princípio diz que características como movimento inercial e não-rotacional são influenciadas pela matéria presente no universo. É importante enfatizar que tal conceito não está presente em teorias anteriores a TRG. Einstein buscou seguir tal princípio, onde algumas idéias de Mach foram incorporadas à TRG.

Na relatividade especial, o elemento de linha do espaço-tempo é dado por:

$$
d s^{2}=d t^{2}-d x^{2}-d y^{2}-d z^{2}
$$

onde $t$ é o tempo e $x, y$ e $z$ são as coordenadas espaciais. Podemos escrever a equação acima de uma forma mais compacta, como mostrado abaixo:

$$
d s^{2}=\eta_{\mu \nu} d x^{\mu} d x^{\nu}
$$

onde $\eta_{\mu \nu}$ é a métrica do espaço-tempo plano e $x^{\mu}$ são as coordenadas espaço-temporais.

Na TRG, as propriedades intrínsecas do espaço-tempo também são dadas por uma métrica. Esta, entretanto, não tem a mesma forma que (1.2), uma vez que ela representa o efeito da gravitação sobre a estrutura do espaço-tempo. O elemento de linha é dado por (Weinberg, 1972): 


$$
d s^{2}=g_{\mu \nu} d x^{\mu} d x^{\nu}
$$

onde $g_{\mu \nu}$ é a métrica que pode representar um espaço-tempo curvo. A presença de matéria no universo influencia a estrutura do espaço-tempo de acordo com as equações de campo de Einstein (Weinberg, 1972):

$$
G_{\mu \nu}-\Lambda g_{\mu \nu}=\chi T_{\mu \nu}
$$

onde $G_{\mu \nu}$ é o tensor de Einstein, $\Lambda$ é a constante cosmológica, $T_{\mu \nu}$ é o tensor de energiamomento e $\chi=\frac{8 \pi G}{c^{4}}$ é a constante de Einstein obtida quando se toma o limite para campos fracos. No tensor de energia-momento devemos considerar todas as fontes de energia e pressão. As equações de campo de Einstein têm o seguinte significado: do lado direito da equação temos as fontes de energia e pressão que curvam o espaço-tempo, já do lado esquerdo temos a geometria que diz como os corpos se movimentam no espaço-tempo. Logo, vemos que a presença de matéria influencia na curvatura do espaço, de acordo com o princípio de Mach.

O tensor de Einstein $G_{\mu \nu}$ pode ser escrito em função do tensor de Ricci $R_{\mu \nu}$, que está ligado às derivadas da métrica $g_{\mu \nu}$, sendo expresso por:

$$
G_{\mu \nu}=R_{\mu \nu}-\frac{1}{2} R g_{\mu \nu}
$$

onde $R=R_{\mu}^{\mu}$ é o escalar de curvatura, obtido através da contração dos índices do tensor de Ricci.

De posse das equações de Einstein, podemos construir modelos cosmológicos. Para isso, devemos especificar as hipóteses básicas do modelo, a fim de determinarmos a métrica, e os componentes do tensor de energia-momento, o que nos permite discutir a dinâmica cósmica.

\subsection{Métrica FLRW (Friedmann-Lemaître-Robertson-Walker)}

A métrica FLRW é obtida através da imposição do Princípio Cosmológico. Este princípio diz que não existem observadores privilegiados no universo. Desta forma, a cada instante $t$, todos os observadores devem ver o mesmo universo, o que implica que o 
universo é homogêneo e isotrópico em grande escala. O elemento de linha para a métrica FLRW, de uma forma geral, é dado por:

$$
d s^{2}=d t^{2}-a(t)^{2}\left[\frac{d r^{2}}{1-k r^{2}}+r^{2} d \theta^{2}+r^{2} \operatorname{sen}^{2} \theta d \phi^{2}\right]
$$

onde $r, \theta$ e $\phi$ são coordenadas esféricas comóveis, $a(t)$ é o fator de escala, que mede distâncias físicas no universo, e $k$ é o parâmetro de curvatura das seções espaciais assumindo os valores: $k=0$ (universo plano), $k=1$ (universo fechado) e $k=-1$ (universo hiperbólico) . Determinamos qual é a dinâmica com a obtenção do fator de escala $a(t)$. Para isso, devemos dizer qual é o tensor de energia-momento e a partir daí resolver as equações de Einstein (1.4).

\subsection{O Redshift}

O redshift é um parâmetro cosmológico observável de suma importância, visto que ele permite determinar a razão entre o fator de escala entre dois instantes distintos. Consideremos um raio de luz que viaja até nós pela direção $-r$, com $\theta$ e $\phi$ fixos. A equação de movimento desse raio é dada por uma geodésica nula, que nesse caso é dada por:

$$
d s^{2}=d t^{2}-a(t)^{2} \frac{d r^{2}}{1-k r^{2}} \equiv 0
$$

Considerando que o raio saiu de uma fonte em $t_{1}, r_{1}, \theta_{1}$ e $\phi_{1}$, ele nos atingirá em $t_{0}$ dado pela equação abaixo:

$$
\int_{t_{1}}^{t_{0}} \frac{d t}{a(t)}=f\left(r_{1}\right),
$$

onde:

$$
f\left(r_{1}\right)=\int_{0}^{r_{1}} \frac{d r}{\sqrt{1-k r^{2}}} .
$$

Suponha também que a próxima crista da onda seja emitida em $t_{1}+\delta t_{1}$, sendo recebida em $t_{0}+\delta t_{0}$. Logo, a equação de movimento é dada por:

$$
\int_{t_{1}+\delta t_{1}}^{t_{0}+\delta t_{0}} \frac{d t}{a(t)}=f\left(r_{1}\right) .
$$


Subtraindo (1.10) de (1.8) e notando que o fator de escala varia pouco entre o tempo típico de emissão entre duas cristas, temos que:

$$
\frac{\delta t_{0}}{a\left(t_{0}\right)}=\frac{\delta t_{1}}{a\left(t_{1}\right)} .
$$

A freqüência observada hoje está relacionada à freqüência emitida pelo inverso da razão entre as diferenças de tempo, logo temos que:

$$
\frac{\nu_{0}}{\nu_{1}}=\frac{a\left(t_{1}\right)}{a\left(t_{0}\right)}
$$

O redshift é definido por:

$$
z=\frac{\lambda_{1}-\lambda_{0}}{\lambda_{1}}
$$

sendo um parâmetro observável, uma vez que podemos relacionar o comprimento de onda de uma linha, obtido espectroscopicamente, com o valor medido no laboratório. Podemos reescrever o redshift em função do fator de escala:

$$
z=\frac{a\left(t_{0}\right)}{a\left(t_{1}\right)}-1
$$

Portanto, vemos que através da medição do redshift da fonte podemos relacionar o fator de escala hoje com o fator de escala no instante em que a luz foi emitida.

\subsection{Tensor de Energia-Momento}

No tensor de energia-momento, devemos inserir todas as componentes do universo, como matéria e radiação. Devido à hipótese de que o universo é homogêneo e isotrópico em grande escala, uma boa descrição dessas componentes é feita supondo que tais podem ser descritas por fluidos perfeitos.

Um fluido perfeito é definido como um fluido sem viscosidade e que não conduz calor em um referencial comóvel. Matematicamente, essa última condição é expressa por $T^{0 i}=$ $T^{i 0}=0$. A componente tempo-tempo expressa a densidade de energia do fluido: $\rho=T^{00}$. Se não há condução de calor e viscosidade, só há fluxo de energia se houver fluxo de partículas. Quando o número de partículas é conservado a evolução do cosmos é adiabática. 
A viscosidade é uma força paralela à interface entre as partículas. Sua ausência implica que as forças são sempre perpendiculares à interface, ou seja, $T^{i j}$ é zero sempre que $i \neq j$. Como a ausência de viscosidade independe da orientação de eixos espaciais, $T^{i j}$ deve ser diagonal em todos referenciais comóveis, ou seja, $T^{i j}=p \delta^{i j}$, onde $p$ é a pressão.

Realizando uma transformação de coordenadas do referencial comóvel para um referencial que se move em relação a ele com velocidade $\vec{v}$, obtemos o tensor de energia-momento para o fluido perfeito de uma forma geral:

$$
T^{\mu \nu}=(\rho+p) u^{\mu} u^{\nu}-p g^{\mu \nu},
$$

onde $u^{\mu}=\delta_{0}^{\mu}$ é a quadrivelocidade dos elementos de volume comóveis para a métrica FLRW. A equação (1.15) está expressa para apenas uma componenente. No caso de várias componentes, basta somarmos suas respectivas densidades de energia e pressões.

\subsection{Dinâmica Cosmológica}

As equações de campo de Einstein (1.4) para a métrica FLRW (1.6) quando consideramos um fluido perfeito para o tensor de energia-momento (1.15) são dadas por:

$$
8 \pi G \rho+\Lambda=3 \frac{\dot{a}^{2}}{a^{2}}+3 \frac{k}{a^{2}}
$$

e:

$$
8 \pi G p-\Lambda=-2 \frac{\ddot{a}}{a}-\frac{\dot{a}^{2}}{a^{2}}-\frac{k}{a^{2}}
$$

onde o ponto representa derivação com relação ao tempo.

As equações acima são conhecidas como equações de Friedmann e nos permitem prever a dinâmica cosmológica. Podemos manipular as equações acima para obtermos a equação que expressa a conservação local de energia:

$$
\dot{\rho}+3 \frac{\dot{a}}{a}(\rho+p)=0
$$

A maioria dos fluidos de interesse cosmológico tem uma equação de estado da forma $p=w \rho$. Logo, a equação (1.18) é facilmente integrável, com solução dada por: 


$$
\rho=\rho_{0}\left(\frac{a_{0}}{a}\right)^{3(1+w)},
$$

onde $\rho_{0}$ é a densidade de energia medida hoje e $a_{0}$ é o fator de escala medido hoje. Podemos agora ver como varia a densidade de energia para alguns fluidos de interesse. A matéria se comporta como poeira, isto é, tem pressão nula. Logo $w=0$ e temos:

$$
\rho_{M}=\rho_{M 0}\left(\frac{a_{0}}{a}\right)^{3}
$$

onde $\rho_{M 0}$ é a densidade de energia da matéria medida hoje. O volume é proporcional a $a(t)^{3}$, e portanto a densidade de energia da matéria é inversamente proporcional ao volume. Para a radiação, $p=\frac{1}{3} \rho$, então sua densidade de energia é dada por:

$$
\rho_{R}=\rho_{R 0}\left(\frac{a_{0}}{a}\right)^{4}
$$

onde $\rho_{R 0}$ é a densidade de energia da radiação medida hoje. Aqui, além da densidade de energia da radiação diminuir com o volume, ainda devemos considerar o fato de que a expansão do universo faz com que os comprimentos de onda sejam afetados, por isso ela cai com $a(t)^{4}$.

Para a constante cosmológica, podemos descrevê-la como um fluido com equação de estado $p=-\rho$, ou seja, $w=-1$. Logo, sua densidade de energia é:

$$
\rho_{\Lambda}=\rho_{\Lambda 0}=\frac{\Lambda}{8 \pi G}
$$

que permanece constante durante a expansão.

É importante também definir os parâmetros observáveis em cosmologia. Primeiramente, definimos o parâmetro de Hubble como a taxa de variação do volume comóvel $V:$

$$
H(t)=\frac{1}{3} \frac{\dot{V}}{V}=\frac{\dot{a}(t)}{a(t)},
$$

onde seu valor medido hoje é conhecido como a constante de Hubble $H_{0}$. Definimos como a densidade crítica:

$$
\rho_{c r}=\frac{3 H^{2}}{8 \pi G} .
$$


Agora podemos definir o parâmetro de densidade para uma componente $i$ como a razão entre a densidade de energia e a densidade crítica:

$$
\Omega_{i}=\frac{\rho_{i}}{\rho_{c r}}
$$

Com as definições adotadas podemos reescrever a equação (1.16), utilizando as definições acima, da seguinte forma:

$$
H(t)^{2}=H_{0}^{2}\left[\Omega_{M 0}\left(\frac{a_{0}}{a(t)}\right)^{3}+\Omega_{R 0}\left(\frac{a_{0}}{a(t)}\right)^{4}+\Omega_{\Lambda}+\Omega_{k 0}\left(\frac{a_{0}}{a(t)}\right)^{2}\right],
$$

onde $\Omega_{k 0}=-\frac{k}{a_{0}^{2} H_{0}^{2}}$ é definido como parâmetro de densidade da curvatura, medido hoje. Tomando a equação acima para o tempo atual, chegamos a um vínculo entre os parâmetros:

$$
\Omega_{M 0}+\Omega_{R 0}+\Omega_{\Lambda}+\Omega_{k 0}=1
$$

Da equação (1.26), podemos ver qual termo domina a expansão para diferentes valores do fator de escala. Quando $a(t)$ é pequeno, vemos que a expansão do universo é dominada pelo termo de radiação. Subseqüentemente, o termo de matéria passa a dominar a expansão, passando pelo termo de curvatura e por fim a constante cosmológica.

Pela análise da equação (1.27) podemos avaliar como a curvatura do espaço está relacionada com o conteúdo de matéria e energia do universo:

$$
\begin{aligned}
& k=0 \Rightarrow \Omega_{M 0}+\Omega_{R 0}+\Omega_{\Lambda}=1 \quad \text { Universo plano, } \\
& k=1 \Rightarrow \Omega_{M 0}+\Omega_{R 0}+\Omega_{\Lambda}>1 \quad \text { Universo fechado, } \\
& k=-1 \Rightarrow \Omega_{M 0}+\Omega_{R 0}+\Omega_{\Lambda}<1 \quad \text { Universo hiperbólico. }
\end{aligned}
$$

Na figura (1.1), vemos como se comporta o fator de escala com o tempo para os três casos, quando o universo é dominado exclusivamente por matéria. Para um universo fechado, vemos que ele atinge um valor máximo de expansão, passando a se contrair e atingir $a(t)=0$, ponto que é chamado de Big Crunch. Para um universo hiperbólico ou plano, ele se expande indefinidamente. 


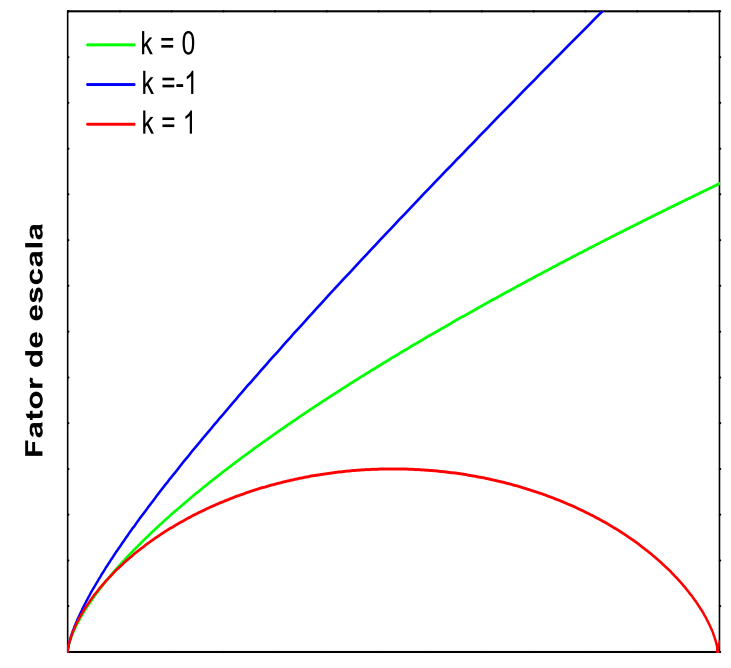

tempo

Figura 1.1: Fator de escala em função do tempo para um universo plano, $k=0$, universo fechado, $k=1$, e universo hiperbólico, $k=-1$. Em todos os casos consideramos um universo dominado somente por matéria.

Por último, definimos o parâmetro de desaceleração do universo, que é dado por:

$$
q(t)=-\frac{a(t) \ddot{a}(t)}{\dot{a}(t)^{2}},
$$

assim definido para que o parâmetro para um universo dominado por matéria seja positivo. Podemos reescrevê-lo em função do parâmetro de densidade, como mostrado abaixo para seu valor medido hoje:

$$
q_{0}=\frac{1}{2} \Omega_{M 0}+\Omega_{R 0}-\Omega_{\Lambda}
$$

logo a combinação dos três parâmetros de densidade pode gerar universos acelerados ou desacelerados, sendo sua medição muito importante para determinarmos qual componente domina a expansão do universo. 
Capítulo 2

\section{A Escada de Distância Cósmica}

\subsection{Introdução}

Neste capítulo estudamos a escada de distância cósmica, onde vemos que para medirmos distâncias cada vez maiores sempre necessitamos calibrar os dados por métodos locais, o que influencia diretamente a determinação dos parâmetros cosmológicos. Primeiramente, falamos sobre os indicadores primários de distância, onde suas luminosidades absolutas são obtidas através de métodos cinemáticos ou indiretamente pela combinação de vários indicadores primários. Embora possam ser estudados diretamente, os indicadores primários dão acesso apenas a objetos em baixos redshifts, da ordem de 0.01, onde os efeitos de curvatura e da expansão do universo podem ser desprezados. Para estudarmos o universo em altos redshifts introduzimos os indicadores secundários de distância, que têm suas luminosidades absolutas determinadas pela calibração em baixos redshifts pelo próprio indicador ou por um indicador primário. Em seguida, descrevemos as distâncias em cosmologia quando consideramos os efeitos da expansão do universo. Por último, falamos dos gamma-ray bursts, que têm sido propostos como velas padrão apenas recentemente. A princípio tais fontes permitem estudar o universo até redshifts da ordem de 7 , sendo seu interesse em cosmologia evidente.

\subsection{Indicadores Primários de Distância}

Os indicadores primários de distância são obtidos através de métodos cinemáticos ou indiretamente pela combinação de indicadores primários que utilizam métodos cinemáticos. Aqui, descrevemos os métodos onde obtemos pequenas distâncias até métodos onde obte- 
mos grandes distâncias, mas que ainda assim não são suficientes para observarmos efeitos cosmológicos. Seguiremos as abordagens apresentadas em Weinberg $(1972,2008)$.

\subsubsection{Paralaxe Trigonométrica}

O movimento da Terra ao redor do Sol faz com que a posição aparente no céu de estrelas próximas mude ao longo do ano quando comparada a estrelas mais distantes. A paralaxe trigonométrica é definida pelo ângulo subentendido pela Terra em sua órbita ao redor do Sol (veja figura 2.1):

$$
p=\frac{d_{T S}}{d}
$$

onde $p$ é geralmente medida em segundos de $\operatorname{arco}, d_{T S}$ é a distância média entre a Terra e o Sol, conhecida como unidade astronômica (UA), com valor igual a $1.496 \times 10^{8} \mathrm{Km}$, e $d$ é a distância entre o Sol e a estrela. Um parsec (pc) é definido como a distância de uma estrela que tem como paralaxe trigonométrica $1^{\prime \prime}$, o que é equivalente a $3.0856 \times 10^{13} \mathrm{Km}$.

\section{Estrela de referência}

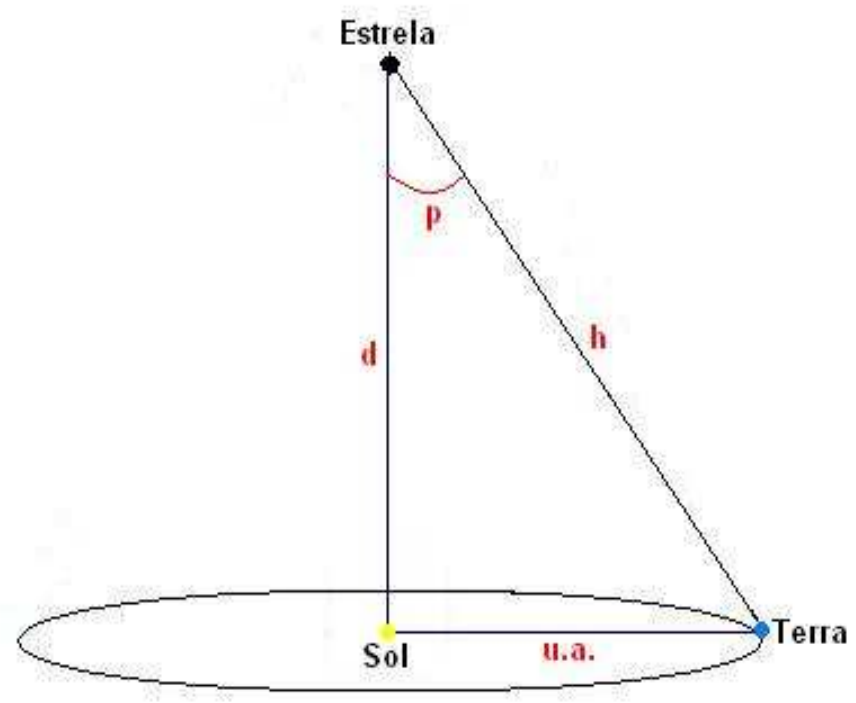

Figura 2.1: Determinação da distância entre o Sol e uma estrela pelo método da paralaxe trigonométrica.

O efeito combinado de extinção e seeing da atmosfera terrestre faz com que paralaxes 
menores que $0.03^{\prime \prime}$ sejam muito difíceis de ser medidas, o que limita a obtenção de distâncias nesse método até 30 parsecs para telescópios terrestres. O número de estrelas com paralaxes determinadas cresceu de forma significativa com o lançamento do satélite Hiparcos, onde 20 mil estrelas tiveram suas paralaxes determinadas com erros não superiores a 10\%, sendo que algumas estão a distâncias superiores a 100 parsecs.

\subsubsection{Movimento Próprio}

O movimento próprio de um objeto é definido como a taxa temporal angular percorrida por este, dada por:

$$
\mu=\frac{v_{p}}{d}
$$

onde $v_{p}$ é a velocidade perpendicular do objeto e $d$ sua distância. Não medimos diretamente a velocidade perpendicular do objeto, somente temos sua velocidade radial através do deslocamento das linhas espectrais pelo efeito Doppler. Logo, a determinação da distância só é possível em alguns casos particulares, onde tal inferência é possível. Podemos citar como exemplo o caso quando em um conjunto de estrelas sabemos as distâncias relativas ou porque todas têm a mesma luminosidade ou porque estão a mesma distância. Esse método só consegue prover resultados na vizinhança solar. Para medirmos maiores distâncias, temos que que adotar novos métodos.

\subsubsection{Luminosidade Aparente}

Podemos determinar a distância a um objeto se soubermos sua luminosidade absoluta e medirmos seu fluxo, também chamado de luminosidade aparente $l$, que é dado pela relação:

$$
l=\frac{L}{4 \pi d^{2}},
$$

onde $L$ é a luminosidade absoluta e $d$ é a distância do objeto.

Em astronomia, devido a origens históricas, utilizamos o termo magnitude para relacionar luminosidade absoluta e aparente. A magnitude bolométrica aparente $m$, onde bolométrica se refere a todos os comprimentos de onda, é definida através do fluxo observado pela seguinte relação: 


$$
l=10^{-\frac{2 m}{5}} \times 2.52 \times 10^{-5} \frac{\mathrm{erg}}{\mathrm{cm}^{2} \mathrm{~S}} .
$$

Já a magnitude absoluta $M$ é definida como a magnitude aparente que um objeto teria se estivesse a uma distância de 10 pc, o que nos dá a seguinte relação:

$$
L=10^{-\frac{2 M}{5}} \times 3.02 \times 10^{35} \frac{\mathrm{erg}}{\mathrm{s}} .
$$

A partir das equações acima podemos expressar a distância de um objeto em função de sua magnitude absoluta e aparente:

$$
d=10^{1+\frac{m-M}{5}} \mathrm{pc}
$$

Existem diversos tipos de estrelas que podemos relacionar algum parâmetro observacional com a luminosidade absoluta, onde descrevemos alguns exemplos. Estrelas que estão em um estágio de queima de hidrogênio em seu núcleo ocupam uma faixa em um diagrama de luminosidade e cor, onde a posição da estrela nesse diagrama depende de sua massa. Essa faixa é conhecida por seqüência principal, sendo o diagrama denominado diagrama H-R (Hertzprung-Russel). As estrelas que estão na seqüência principal obedecem a uma relação entre cor e luminosidade, onde tais parâmetros dependem da massa. Para calibrar a relação, observam-se várias estrelas que estão na seqüência principal em aglomerados, onde supomos que todas estão à mesma distância. Com o lançamento do satélite Hiparcos, essa calibração foi melhorada pela utilização de paralaxes trigonométricas de estrelas na seqüência principal medidas em vários aglomerados. Assim sendo, conseguimos medir distâncias de estrelas que estão na seqüência principal até distâncias da ordem de $10^{5} \mathrm{pc}$.

Outro exemplo são as estrelas variáveis RR Lyrae, que possuem um período entre 0.2 e 0.8 dias. Utilizando métodos cinemáticos foi obtida sua magnitude absoluta, onde se observou que ela é aproximadamente constante. Logo, uma vez que reconhecemos esse tipo de estrela pelo seu curto período de pulsação, podemos calcular sua distância pela medição de sua magnitude aparente. Visto que essas estrelas não são muito brilhantes, podemos obter distâncias até da ordem de $3 \times 10^{5} \mathrm{pc}$.

Outro tipo de estrela variável são as estrelas cefeidas, cujo nome se deve a primeira estrela detectada desse tipo, a $\delta$ Chephei. Elas têm um período entre 2 e 45 dias. Em 1912 
Henrieta Leavitt descobriu que estrelas cefeidas na Pequena Nuvem de Magalhães apresentavam uma relação entre período e luminosidade, mas a distância não era conhecida. Com a determinação da luminosidade absoluta das cefeidas em aglomerados abertos na Galáxia, foi possível calibrar a relação. Esse fato permitiu que Hubble em 1923 determinasse a distância a M31 e assim foi possível concluir que tal objeto constitui na verdade uma outra galáxia, dessa forma iniciando a chamada astronomia extragaláctica. De modo análogo ao utilizado para as estrelas RR Lyrae, medimos a magnitude aparente e o período da cefeida, o que nos permite obter a luminosidade absoluta e assim sua distância. Devido ao seu grande brilho, podemos determinar distâncias utilizando cefeidas até da ordem de $3 \times 10^{7}$ pc. Para atingirmos distâncias ainda maiores, precisamos dos indicadores secundários de distância, que são o tema da próxima seção.

\subsection{Indicadores Secundários de Distância}

Para determinarmos distâncias em redshifts onde a velocidade de expansão do universo não é desprezível temos que usar indicadores secundários de distância. Para tais distâncias, precisamos de objetos muito luminosos, como uma galáxia. Com o lançamento do Hubble Space Telescope (HST), podemos observar cefeidas até distâncias da ordem de $30 \mathrm{Mpc}$, o que nos permite calibrar tais indicadores a partir das cefeidas. Nesta seção descrevemos quatro indicadores secundários que estão bem estabelecidos na literatura.

\subsubsection{A Relação de Tully-Fisher}

Podemos estimar a luminosidade absoluta de certas galáxias espirais a partir do alargamento de suas linhas de absorção em 21 cm, devido à rotação da galáxia. A largura da linha nos permite estimar a velocidade de rotação máxima da galáxia, que está relacionada com sua massa total que por sua vez está relacionada com a luminosidade total. Ela é conhecida como relação de Tully-Fisher (Tully e Fisher, 1977). A relação entre a luminosidade e a largura da linha $\Delta V$ é dada por (Combes et al., 2002):

$$
L=k \Delta V^{\alpha}
$$

onde $k$ é uma constante de proporcionalidade e $\alpha \sim 4$. Pela medição da magnitude aparente 
e da largura da linha é possível calcular a distância da galáxia em questão.

\subsubsection{A Relação de Faber-Jackson}

A relação de Faber-Jackson (Faber e Jackson, 1976) provém da correlação da dispersão de velocidades com a luminosidade absoluta de galáxias espirais. Embora tenha sido obtida empiricamente, essa relação pode ser explicada teoricamente utilizando o teorema do virial, que relaciona a velocidade quadrática média com a massa da galáxia. A relação é expressa por (Combes et al., 2002):

$$
L=k \sigma^{4}
$$

onde $k$ é uma constante de proporcionalidade e $\sigma$ é a dispersão de velocidades.

\subsubsection{O Plano Fundamental}

Esse método melhora a relação de Faber-Jackson, uma vez que ele leva em consideração que a correlação também depende do brilho superficial da galáxia, logo depende de sua área. Esse método foi proposto por Dressler et al. (1987) e Djorgovski e Davis (1987). Levando em consideração o brilho superficial $\langle S B\rangle$, a relação é dada por (Djorgovski e Davis, 1987):

$$
L \sim \sigma^{3.45}<S B>^{-0.86}
$$

Pela medição da magnitude aparente, da dispersão de velocidades e do brilho superficial podemos inferir a distância do objeto.

\subsubsection{Supernovas Tipo Ia}

Uma supernova tipo Ia ocorre quando uma anã branca em um sistema binário acreta massa até chegar próximo ao limite de Chandrasekhar, que é a maior massa possível que uma estrela pode suportar pela pressão degenerada de elétrons (Hoyle e Fowler, 1960). Quando o limite está próximo de ser atingindo, a estrela torna-se instável, a temperatura e densidade aumentam, criando condições para a queima de carbono e oxigênio em níquel, o que gera uma explosão que tem brilho equivalente a de uma galáxia inteira. Como todas 
as estrelas explodem com máxima próxima ao limite de Chandrasekhar, elas têm luminosidades absolutas muito próximas, cuja variação está relacionada com a altura do pico da curva de luz e o tempo de decaimento (Phillips, 1993). Assim sendo, podemos utilizá-las como velas padrão e, pela possibilidade de medí-las em altos redshifts, têm um papel importante na determinação dos parâmetros cosmológicos. Até o presente, supernovas tipo Ia foram detectadas até o redshift $z \sim 2$.

\subsection{Medidas de Distância em Espaço-Tempo Curvo}

Quando vamos medir distâncias em altos redshifts devemos levar em consideração os efeitos da expansão do universo. Nessa escala a distância não é um invariante, o que nos faz definí-la a partir do modo como são realizadas as observações. Em cosmologia existem duas distâncias muito utilizadas: a distância de luminosidade e a distância de diâmetro angular, que são discutidas abaixo.

\subsubsection{Distância de Luminosidade}

Quando propomos a equação (2.3), vimos que a luminosidade aparente é inversamente proporcional à área. Para um universo em expansão, no instante $t_{0}$ em que a luz atinge a Terra, a área própria em torno do objeto que passa pela Terra é $4 \pi r_{1}^{2} a\left(t_{0}\right)^{2}$, onde $r_{1}$ é a coordenada radial do objeto. Assim, comparando o termo obtido com a equação (2.3), temos que substituir o termo $1 / d^{2}$ por $r_{1}^{2} a\left(t_{0}\right)^{2}$. Além disso, a taxa em que os fótos chegam a Terra cai com um fator de $1+z$. O mesmo ocorre para a energia dos fótons. Logo, chegamos a seguinte equação que leva em conta os efeitos da expansão:

$$
l=\frac{L}{4 \pi r_{1}^{2} a\left(t_{0}\right)^{2}(1+z)^{2}} .
$$

Comparando as equações (2.3) e (2.10), definimos a distância de luminosidade como:

$$
D_{L}=a\left(t_{0}\right) r_{1}(1+z)
$$

Podemos expandir a expressão acima em uma série de potências em $z$, na seguinte forma: 


$$
D_{L}=H_{0}^{-1}\left[z+\frac{1}{2}\left(1-q_{0}\right) z^{2}+\ldots\right]
$$

Logo, a utilização de velas padrão nos permite obter a constante de Hubble e o parâmetro de desaceleração.

\subsubsection{Distância de Diâmetro Angular}

A distância de diâmetro angular vem da relação entre o tamanho angular de um objeto e seu tamanho intrínseco. Consideremos um objeto de tamanho intrínseco $D$ com coordenada radial $r_{1}$, que apresenta um tamanho angular $\theta$. Pela simples integração da equação (1.6), assumindo que a luz foi emitida em um instante $t_{1}$, temos que:

$$
D=a\left(t_{1}\right) r_{1} \theta
$$

Definimos a distância de diâmetro angular $D_{A}$ por analogia a geometria euclideana:

$$
D_{A}=\frac{D}{\theta}
$$

o que nos leva a:

$$
D_{A}=a\left(t_{1}\right) r_{1}
$$

Pela comparação da equação acima com a equação (2.11), vemos que ambas estão relacionadas por:

$$
\frac{D_{L}}{D_{A}}=(1+z)^{2},
$$

onde vemos que para $z<<1$ ambas as distâncias são equivalentes, conforme o esperado.

A utilização da distância de diâmetro angular necessita de fontes astrofísicas que possam ser consideradas réguas padrão, ou seja, fontes que tenham o mesmo comprimento intrínseco. Estudos têm sido realizados pela utilização de fontes de rádio ultracompactas, tanto pela consideração que tais fontes não devem sofrer efeitos evolutivos (Kellermann, 1993), ou pela consideração que o tamanho evolua como uma lei de potência do tipo $D=D_{0}(1+z)^{\alpha}$ (Buchalter et al., 1998; Gurvits et al., 1999). 


\subsection{Gamma-Ray Bursts}

Os chamados gamma-ray bursts são emissões curtas e intensas de raios gama (0.01 - $1 \mathrm{MeV}$ ) que têm fascinado astrônomos e cosmólogos desde sua inesperada detecção no final da década de 60 por satélites do tipo Vela e, finalmente, anunciados em 1973, por Klebesadel et al. (1973). Os eventos, observados uma ou duas vezes por dia, chegam de todas as direções no céu e sua duração varia entre dezenas de milisegundos até milhares de segundos. Seu mecanismo físico tem sido longamente debatido (Damour e Ruffini, 1975; Cavallo e Rees, 1978; Ruffini et al., 2003; Piran, 2004; Fan e Piran, 2008) principalmente devido à dificuldade de se determinar sua exata posição e distância, mas sua origem cosmológica foi finalmente confirmada (Metzger et al., 1997; Costa et al., 1997; van Paradijs et al., 1997; Frail et al., 1997).

Uma das interpretações mais aceitas para os gamma-ray bursts é o chamado modelo da bola de fogo (fireball model, ver figura 2.2). Em tal modelo os raios gama (primários) são devidos a jatos ultra-relativísticos com fatores de Lorentz variáveis (extremamente altos) e ejetados por um engenho central, enquanto os chamados afterglows são produzidos na interação entre o material ejetado e o meio (choques reversos e externos). Os mecanismos de radiação mais discutidos incluem emissão síncrotron e espalhamento Compton inverso. Ambos são capazes de produzir emissão eletromagnética num largo intervalo de energia, ou seja, desde a faixa de rádio até raios gama duros (escala de $\mathrm{GeV}$ ). O engenho central é uma denominação genérica para as possíveis fontes compactas que geram os jatos ultrarelativísticos (buracos negros, supernovas, coalescência de estrelas de nêutrons, etc).

Nesta seção, apresentamos a utilização de gamma-ray bursts como velas padrão. Uma ênfase especial é dirigida para o método de calibração originalmente proposto por Schaefer (2007) e sua posterior utilização por Mosquera Cuesta et al. (2008). Finalmente, descrevemos a calibração de GRBs pela utilização de supernovas Ia.

Embora não exista um modelo físico atualmente aceito para explicar os GRBs, são conhecidas diversas propriedades espectrais e de sua curva de luz, o que permite calcular sua luminosidade absoluta. Conhecendo sua luminosidade, podemos utilizá-los como velas padrão. O processo é o mesmo utilizado anteriormente. Primeiramente calibramos o indicador de luminosidade absoluta. Depois a distância é inferida pela relação (2.3) a partir da medida de sua luminosidade aparente. Como não existem GRBs em baixos 


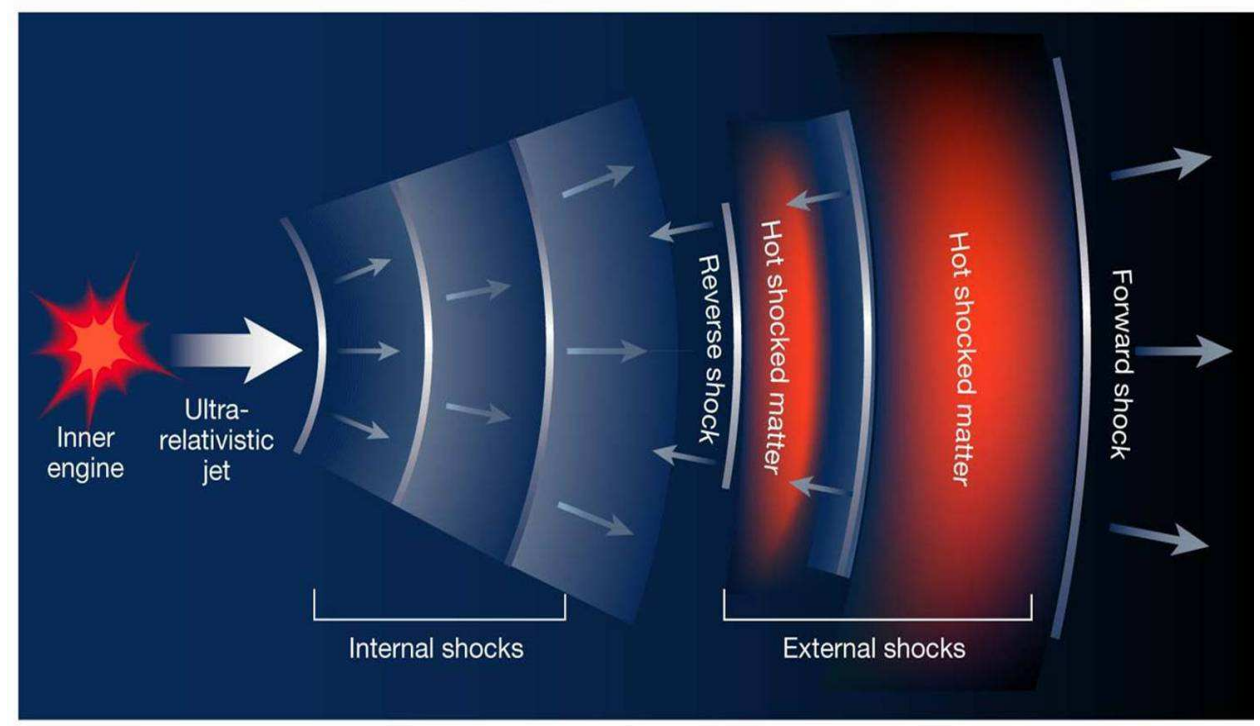

Figura 2.2: Modelo da bola de fogo (fireball model). A figura, retirada de Piran (2003), mostra esquematicamente o engenho central, o jato ultra-relativístico e as regiões de choque (interna e externa) que geram os afterglows.

redshifts, a calibração dos indicadores de luminosidade depende do modelo cosmológico adotado. É sobre esse tema que tratam as próximas seções, onde discutimos a calibração para o modelo $\Lambda$ CDM, para o modelo cardassiano e por fim a utilização de supernovas Ia para realizar a calibração.

\subsubsection{Método de Calibração de Schaefer}

Schaefer (2007) compilou cinco relações fenomenológicas observadas em gamma-ray bursts (GRBs) afim de utilizá-las para vincular parâmetros cosmológicos. São elas: $\tau_{l a g}-L$, $V-L, E_{\text {peak }}-L, E_{\text {peak }}-E_{\gamma}$ e $\tau_{R T}-L$, onde L é a luminosidade isotrópica emitida pelo objeto, $\tau_{l a g}$ é o tempo de atraso entre a curva de luz de fótons com altas energias e fótons menos energéticos, $\mathrm{V}$ é a variabilidade da curva de luz, $E_{\text {peak }}$ representa o ponto onde se concentra a maior emissão de energia, $E_{\gamma}$ é a energia total emitida em raios gama pelo objeto e $\tau_{R T}$ é o tempo mínimo que a curva de luz leva para atingir metade do pico de energia. A luminosidade está relacionada com a distância de luminosidade pela seguinte expressão (equivalente a (2.10)):

$$
L=4 \pi D_{L}^{2} P_{b o l o}
$$


onde $P_{\text {bolo }}$ é o fluxo bolométrico medido. Como $D_{L}$ depende dos parâmetros cosmológicos, vemos que qualquer relação fenomenológica entre a luminosidade e um observável do GRB dependerá da cosmologia adotada, isso porque não existem GRBs em baixos redshifts para realizarmos uma calibração independente da cosmologia. O mesmo ocorre para a relação $E_{\text {peak }}-E_{\gamma}$, pois $E_{\gamma}$ também depende de $D_{L}$ através da seguinte relação (Schaefer, 2007):

$$
E_{\gamma}=\left(1-\cos \theta_{\text {jet }}\right) 4 \pi D_{L}^{2} S_{\text {bolo }}(1+z)^{-1},
$$

onde $\theta_{\text {jet }}$ é o ângulo de abertura do jato e $S_{b o l o}$ é a intensidade bolométrica dos raios gama.

Para testes cosmológicos, temos que realizar a calibração para cada cosmologia de interesse. Entretanto, tal fato pode ser amenizado caso a calibração dependa apenas fracamente da cosmologia utilizada, o que permite utilizar a mesma calibração para analisar diferentes cosmologias.

As relações fenomenológicas são representadas por leis de potência da forma $L=a X^{b}$, onde X é o observável em questão. O procedimento utilizado é simplesmente realizar um ajuste $\log -\log$ aos dados. Como ambos os lados da equação são independentes, isto é, o valor de L não é originado diretamente do valor de $\mathrm{X}$, foi utilizado para o ajuste o método da bissetriz de dois mínimos quadrados comuns (Isobe et al., 1990). Também foram desconsiderados os erros, uma vez que o espalhamento obtido é grande e pode ser causado por um erro sistemático, que foi estimado considerando-se o melhor ajuste possível tomando o valor de $\chi^{2}=1$ após realizado o ajuste. Para a calibração foi considerado como modelo fiducial o $\Lambda$ CDM plano com $\Omega_{M}=0.27$ e $H_{0}=72 \mathrm{Km} \mathrm{s}^{-1} \mathrm{Mpc}^{-1}$. Os valores obtidos para as calibrações com suas respectivas análises constituem o objetivo das próximas seções.

$$
\tau_{\text {lag }}-L
$$

O $\tau_{\text {lag }}$ de um GRB é o tempo de atraso entre a curva de luz de fótons com altas energias e fótons menos energéticos, ou mais especificamente, o tempo de atraso dos picos de energia nas bandas de 100 a $300 \mathrm{KeV}$ e 25 a $50 \mathrm{KeV}$ por exemplo. Devido à dificuldades observacionais, uma definição prática pode ser tomada definindo o $\tau_{l a g}$ como a máxima correlação entre as curvas. Também temos que levar em consideração a dilatação do tempo, onde divide-se o tempo obtido por $1+z$ para se obter o tempo no referencial do GRB. O ajuste obtido foi: 


$$
\log L=52.26-1.01 \log \left[\frac{\tau_{l a g}(1+z)^{-1}}{0.1 s}\right],
$$

onde o valor de $0.1 \mathrm{~s}$ foi adotado para minimizar a correlação entre a constante de normalização e o expoente durante o ajuste, mas não afeta o valor obtido dos parâmetros nem suas incertezas. As incertezas para $a=52.26$ e $b=-1.01$ são: $\sigma_{a}=0.06$ e $\sigma_{b}=0.05$. Tomando o valor de $\chi^{2}$ reduzido igual a um, podemos estimar o valor do erro sistemático, que foi de $\sigma_{s i s}=0.39$. A incerteza do logaritmo da luminosidade é calculada utilizando propagação de erros:

$$
\sigma_{\log L}^{2}=\sigma_{a}^{2}+\left\{\sigma_{b} \log \left[\frac{\tau_{l a g}(1+z)^{-1}}{0.1 s}\right]\right\}^{2}+\left(\frac{0.4343 b \sigma_{\tau_{l a g}}}{\tau_{l a g}}\right)^{2}+\sigma_{\text {sis }}^{2} .
$$

$V-L$

A variabilidade de um GRB é uma medida de quão lisa ou pontiaguda é sua curva de luz. Uma grande quantidade de definições de V é possível, então Schaefer utilizou aquela que minimizou o espalhamento na correlação entre a variabilidade e a luminosidade, tendo obtido o seguinte ajuste:

$$
\log L=52.49+1.77 \log \left[\frac{V(1+z)}{0.02}\right],
$$

onde novamente foi escolhido o valor de 0.02 para minimizar a correlação entre a constante de normalização e o expoente durante o ajuste. Note que o valor de V observado varia inversamente ao tempo, devemos então multiplicar por $1+z$ para transformar para o referencial do GRB. As incertezas obtidas foram de: $\sigma_{a}=0.22, \sigma_{b}=0.12$ e $\sigma_{\text {sis }}=0.40$, sendo a incerteza do logaritmo da luminosidade dada por:

$$
\sigma_{\log L}^{2}=\sigma_{a}^{2}+\left\{\sigma_{b} \log \left[\frac{V(1+z)}{0.02}\right]\right\}^{2}+\left(\frac{0.4343 b \sigma_{V}}{V}\right)^{2}+\sigma_{\text {sis }}^{2}
$$

$E_{\text {peak }}-L$

$E_{\text {peak }}$ representa o ponto onde se concentra a maior emissão de energia do GRB, estando relacionada com a luminosidade pela física que ocorre durante a emissão do pico de energia. Devemos multiplicar o valor de $E_{\text {peak }}$ por $1+z$ para corrigir o redshift do espectro. O ajuste obtido foi de: 


$$
\log L=52.21+1.68 \log \left[\frac{E_{\text {peak }}(1+z)}{300 \mathrm{KeV}}\right],
$$

onde novamente foi escolhido o valor de $300 \mathrm{KeV}$ para minimizar a correlação entre a constante de normalização e o expoente durante o ajuste. Para esta relação, as incertezas obtidas foram $\sigma_{a}=0.13, \sigma_{b}=0.05$ e $\sigma_{\text {sis }}=0.36$. De maneira análoga ao obtido anteriormente, a incerteza do logaritmo da luminosidade é:

$$
\sigma_{\log L}^{2}=\sigma_{a}^{2}+\left\{\sigma_{b} \log \left[\frac{E_{\text {peak }}(1+z)}{300 \mathrm{KeV}}\right]\right\}^{2}+\left(\frac{0.4343 b \sigma_{E_{p e a k}}}{E_{\text {peak }}}\right)^{2}+\sigma_{\text {sis }}^{2} .
$$

$E_{\text {peak }}-E_{\gamma}$

$E_{\gamma}$ é a energia total emitida em raios gama pelo objeto e sua origem física pode ser entendida dentro do modelo padrão de jatos (Eichler e Levinson, 2004; Yamazaki et al., 2004; Rees e Mészáros, 2005; Levinson e Eichler, 2005). Para transformar essa relação para o referencial do GRB, devemos multiplicar por $1+z$ o valor de $E_{\text {peak }}$ e o valor de $E_{\gamma}$ já foi corrigido pela equação (2.18). Essa relação é a que apresenta o menor espalhamento, com o ajuste dado por:

$$
\log E_{\gamma}=50.57+1.63 \log \left[\frac{E_{\text {peak }}(1+z)}{300 \mathrm{KeV}}\right],
$$

onde novamente foi escolhido o valor de $300 \mathrm{KeV}$ para minimizar a correlação entre a constante de normalização e o expoente durante o ajuste. Para esta relação, as incertezas obtidas foram $\sigma_{a}=0.09, \sigma_{b}=0.03$ e $\sigma_{\text {sis }}=0.16$. A incerteza do logaritmo de $E_{\gamma}$ é dada por:

$$
\sigma_{\log E_{\gamma}}^{2}=\sigma_{a}^{2}+\left\{\sigma_{b} \log \left[\frac{E_{\text {peak }}(1+z)}{300 \mathrm{KeV}}\right]\right\}^{2}+\left(\frac{0.4343 b \sigma_{E_{\text {peak }}}}{E_{\text {peak }}}\right)^{2}+\sigma_{\text {sis }}^{2}
$$

$\tau_{R T}-L$

$\tau_{R T}$ é o tempo mínimo que a curva de luz leva para atingir metade do pico de energia. Sua origem física pode estar relacionada com um jato que se choca. Devemos transformar tal relação para o referencial do GRB, logo temos que dividir o tempo obtido por $1+z$ para levarmos em consideração o efeito da dilatação do tempo. O ajuste obtido é dado por: 


$$
\log L=52.54-1.21 \log \left[\frac{\tau_{R T}(1+z)^{-1}}{0.1 s}\right],
$$

onde novamente foi escolhido o valor de 0.01 s para minimizar a correlação entre a constante de normalização e o expoente durante o ajuste. Para esta relação, as incertezas obtidas foram $\sigma_{a}=0.06, \sigma_{b}=0.06$ e $\sigma_{\text {sis }}=0.47$. Para o logaritmo da luminosidade temos a seguinte incerteza:

$$
\sigma_{\log L}^{2}=\sigma_{a}^{2}+\left\{\sigma_{b} \log \left[\frac{\tau_{R T}(1+z)^{-1}}{0.1 s}\right]\right\}^{2}+\left(\frac{0.4343 b \sigma_{\tau_{R T}}}{\tau_{R T}}\right)^{2}+\sigma_{\text {sis }}^{2} .
$$

Uma compilação dos resultados obtidos para as cinco calibrações é apresentada na tabela 2.1 .

Tabela 2.1 - Resultados da calibração de Schaefer (2007).

\begin{tabular}{lccccc}
\hline \hline & $\tau_{\text {lag }}-L$ & $V-L$ & $E_{\text {peak }}-L$ & $E_{\text {peak }}-E_{\gamma}$ & $\tau_{R T}-L$ \\
\hline$a$ & 52.26 & 52.49 & 52.21 & 50.57 & 52.54 \\
$\sigma_{a}$ & 0.06 & 0.22 & 0.13 & 0.09 & 0.06 \\
$b$ & -1.01 & 1.77 & 1.68 & 1.63 & -1.21 \\
$\sigma_{b}$ & 0.05 & 0.12 & 0.05 & 0.03 & 0.06 \\
$\sigma_{\text {sis }}$ & 0.39 & 0.40 & 0.36 & 0.16 & 0.47 \\
& & & & & \\
\hline
\end{tabular}

\section{Módulo de Distância}

A partir das relações (2.17) e (2.18), podemos obter o módulo de distância, que é simplesmente a diferença entre a magnitude aparente e a magnitude absoluta, a partir da seguinte relação:

$$
\mu=5 \log \left(D_{L}\right)+25
$$

onde $D_{L}$ é dada em Mpc (Peebles, 1993). A incerteza para a relação $E_{\text {peak }}-E_{\gamma}$, obtida através da fórmula de propagação de erros padrão, é dada por: 


$$
\sigma_{\mu}=\left[\left(2.5 \sigma_{\log E_{\gamma}}\right)^{2}+\left(\frac{1.086 \sigma_{S_{\text {bolo }}}}{S_{\text {bolo }}}\right)^{2}+\left(\frac{1.086 \sigma_{F_{\text {beam }}}}{F_{\text {beam }}}\right)^{2}\right]^{\frac{1}{2}}
$$

onde $F_{\text {beam }}=1-\cos \theta_{\text {jet }}$. Para as outras relações a incerteza assume a seguinte forma:

$$
\sigma_{\mu}=\left[\left(2.5 \sigma_{\log L}\right)^{2}+\left(\frac{1.086 \sigma_{P_{\text {bolo }}}}{P_{\text {bolo }}}\right)^{2}\right]^{\frac{1}{2}} .
$$

Agora podemos combinar as distâncias obtidas afim de chegarmos a um valor que melhor represente o módulo de distância do objeto. Como cada relação apresenta uma dispersão diferente, consideramos a média ponderada dos módulos:

$$
\mu=\frac{1}{\Sigma_{i} \sigma_{\mu_{i}}^{-2}} \sum_{i}\left(\frac{\mu_{i}}{\sigma_{\mu_{i}}^{2}}\right)
$$

da mesma forma para a incerteza:

$$
\sigma_{\mu}=\left(\sum_{i} \sigma_{\mu_{i}}^{-2}\right)^{\frac{1}{2}}
$$

onde a soma em $i$ cobre os dados existentes para cada objeto. De posse do módulo de distância obtido através dos dados observacionais podemos fazer o teste estatístico $\chi^{2}$ para vincular os parâmetros cosmológicos, uma vez que a distância de luminosidade depende desses parâmetros.

\subsubsection{Calibração para o Modelo Cardassiano}

O modelo cardassiano é um modelo cosmológico plano, composto só por matéria e que acelera em baixos redshifts (Freese e Lewis, 2002). Esse modelo cosmológico é obtido somando-se um termo não linear ao parâmetro de Hubble. Tal termo pode ser interpretado via teoria de branas (Freese e Lewis, 2002) ou por um fluido exótico (Gondolo e Freese, 2003). Assim, o parâmetro de Hubble é dado por:

$$
H^{2}=\frac{8 \pi G \rho}{3}\left[1+\left(\frac{\rho_{\text {card }}}{\rho}\right)^{(1-n)}\right]
$$

onde $\rho_{\text {card }}$ é a densidade a partir da qual o segundo termo passa a dominar a expansão e $n$ é um parâmetro fenomenológico que deve ser obtido através dos dados observacionais. 
Mosquera Cuesta et al. (2008) utilizou o mesmo método desenvolvido por Schaefer (2007) para este modelo. Os resultados para as cinco calibrações estão listados na tabela 2.2 .

Para a calibração foi tomado como modelo fiducial o modelo com $\Omega_{M}=0.27, H_{0}=$ $72 \mathrm{Km} \mathrm{s}^{-1} \mathrm{Mpc}^{-1}$ e $n=0.2$.

Tabela 2.2 - Resultados da calibração para o modelo cardassiano.

\begin{tabular}{lccccc}
\hline \hline & $\tau_{\text {lag }}-L$ & $V-L$ & $E_{\text {peak }}-L$ & $E_{\text {peak }}-E_{\gamma}$ & $\tau_{R T}-L$ \\
\hline$a$ & 52.23 & 52.43 & 52.18 & 50.52 & 52.48 \\
$\sigma_{a}$ & 0.07 & 0.07 & 0.05 & 0.05 & 0.07 \\
$b$ & -1.00 & 1.77 & 1.68 & 1.68 & -1.21 \\
$\sigma_{b}$ & 0.09 & 0.19 & 0.10 & 0.10 & 0.11 \\
$\sigma_{\text {sis }}$ & 0.36 & 0.47 & 0.40 & 0.21 & 0.47 \\
& & & & & \\
\hline
\end{tabular}

Comparando os resultados apresentados nas tabelas 2.1 e 2.2 vemos que, embora os valores ajustados para as calibrações estejam próximos para estes dois modelos fiduciais, devemos analisar de forma mais quantitativa sua influência. Para isso, analisamos os vínculos cosmológicos utilizando as duas calibrações. Existem outros métodos de calibração, dentre os quais utilizar supernovas do tipo Ia em baixos redshifts para calibrar as relações fenomenológicas dos GRBs (Kodama et al., 2008), cujo método discutimos abaixo.

\subsubsection{Calibração utilizando Supernovas Ia}

Este método foi proposto por Kodama et al. (2008). Ele consiste em utilizar supernovas para calibrar a relação $E_{\text {peak }}-L$, também conhecida como relação de Yonetoku (Yonetoku et al., 2004), para a região onde ambas amostras existem, e aplicar o resultado para os GRBs com maiores redshifts.

A utilização das supernovas é proveniente da fórmula empírica obtida por Riess et al. (2007) para o intervalo $0.359<z<1.755$ :

$$
\frac{D_{L}(z)}{10^{27} \mathrm{~cm}}=14.57 z^{1.02}+7.16 z^{1.76}
$$


Essa equação tem como única suposição que supernovas podem ser consideradas velas padrão. A calibração obtida para a relação de Yonetoku foi de:

$$
\left(\frac{L}{10^{52} \mathrm{erg} s^{-1}}\right)=(1.31 \pm 0.67) \times 10^{-4}\left(\frac{E_{\text {peak }}(1+z)}{1 \mathrm{KeV}}\right)^{1.68 \pm 0.09},
$$

onde um erro sistemático de $9.57 \times 10^{-5}$ foi obtido. Feita a calibração, calculamos a distância de luminosidade para os outros objetos:

$$
D_{L}(z)=10^{24} \mathrm{~cm} \sqrt{\frac{1.31}{4 \pi l}}\left[E_{\text {peak }}(1+z)\right]^{1.68 / 2} .
$$

Uma vez obtida a distância de luminosidade, utilizamos a relação (2.29) para calcular a módulo de distância. Essa calibração também pode ser usada para vincular os parâmetros cosmológicos, mas não a utilizamos nesta dissertação. 
Capítulo 3

\section{Cosmologias Aceleradas}

\subsection{Introdução}

O modelo cosmológico padrão até meados da década de 90 era o de Einstein-de Sitter, como previsto pela inflação, composto de matéria e radiação com constante cosmológica nula. A visão do cosmos mudou abruptamente em 1998 quando dois grupos independentes, o Supernova Cosmology Project e o High-z Supernova Search, concluíram que o estágio atual da expansão do universo é acelerado (Riess et al., 1998; Perlmutter, 1999). Tal descoberta foi uma conseqüência da utilização de supernovas do tipo Ia como velas padrão. Dentro da TRG, tal fato é usualmente interpretado como uma evidência da existência de um fluido exótico com pressão negativa chamado de energia escura, sendo a constante cosmológica (ou energia do vácuo) a candidata mais simples.

O grupo do Supernova Cosmology Project estudou 42 supernovas com redshifts entre 0.18 e 0.83 , juntamente com um conjunto de supernovas com redshifts menores que 0.1. Eles encontraram para um modelo composto de matéria escura e constante cosmológica que $\Omega_{\Lambda} \leq 0$ está excluído com confiança estatística de $99 \%$, independente da curvatura da seção espacial. Para um modelo plano, o melhor ajuste foi de $\Omega_{M}=0.28$, o que resulta em um parâmetro de desaceleração $q_{0}=-0.58$, indicando que o universo está em uma fase de expansão acelerada. Os resultados obtidos estão apresentados na figura 3.1.

Já o grupo do High-z Supernova Search estudou 16 supernovas com redshifts entre 0.16 e 0.97, incluindo duas supernovas do Supernova Cosmology Project, e 34 supernovas com baixos redshifts, chegando a conclusão de que para um modelo com matéria escura e constante cosmológica temos que $\Omega_{\Lambda}>0$ com um nível de confiança estatística de $99.7 \%$, independente da curvatura da seção espacial. O melhor ajuste para um modelo plano foi de 


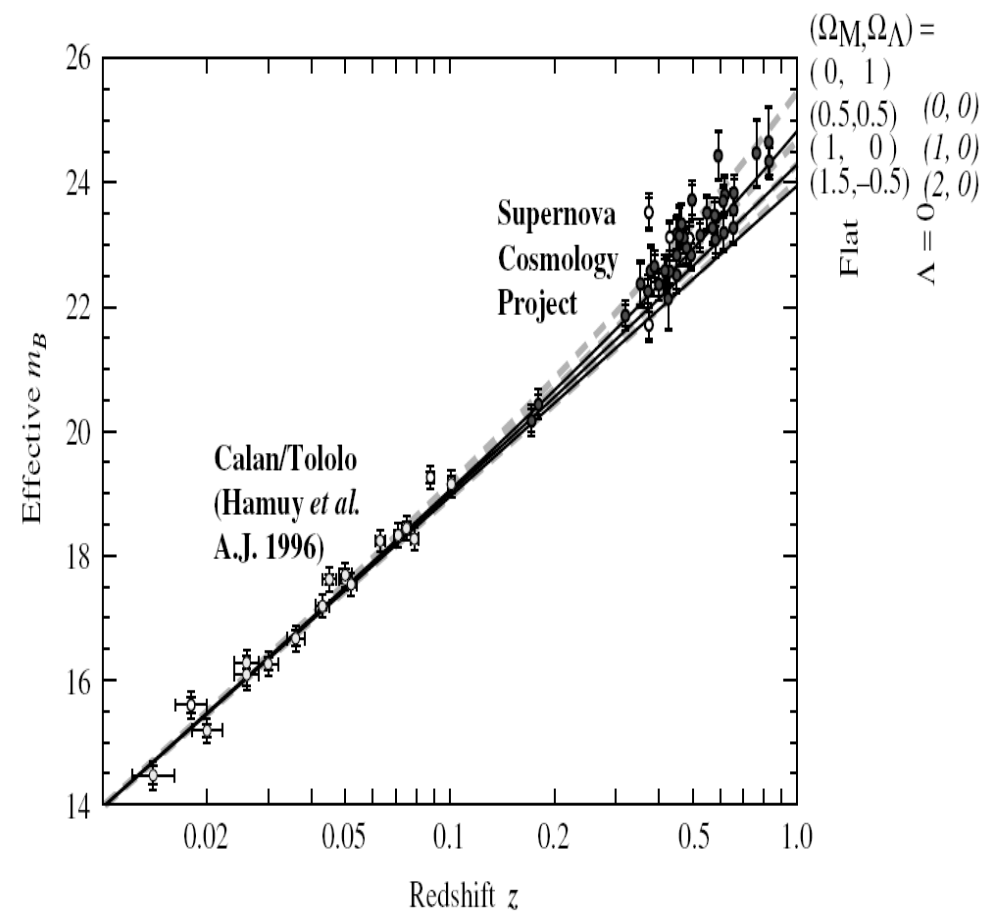

Figura 3.1: Evidência da aceleração do universo obtida com 42 supernovas observadas pelo Supernova Cosmology Project e 18 supernovas do Cálan-Tololo Supernovae Survey (Hamuy et al., 1996).

$\Omega_{M}=0.28$. Supondo $\Omega_{M}>0$, eles obtiveram $q_{0}<0$ com um nível de confiança estatística de $95 \%$. Os resultados obtidos estão apresentados na figura 3.2.

Afora a evidência vinda de supernovas, existem outros fatores que nos levam a inferir a existência da energia escura. Entre eles, podemos citar: as anisotropias do espectro de potência da radiação cósmica de fundo (Komatsu et al., 2008), estruturas em grande escala (Cole et al., 2005; Tegmark et al., 2004), observações em raios X de aglomerados de galáxias (Lima et al., 2003), objetos velhos em altos redshifts (Krauss, 1997; Alcaniz e Lima, 1999b), tamanho angular de fontes de rádio compactas (Gurvits et al., 1999; Lima e Alcaniz, 2002) e rádio-galáxias (Daly e Guerra, 2002), gamma-ray bursts (Schaefer, 2007). Na figura 3.3, mostramos a genealogia da energia escura.

Descrevemos a seguir os principais candidatos à energia escura. Apresentamos também modelos relativísticos onde tal componente não é necessária: o modelo cardassiano e o modelo com criação de matéria. O primeiro deles é inspirado em teorias de cordas e membranas, enquanto o segundo é formulado no contexto da relatividade geral. 


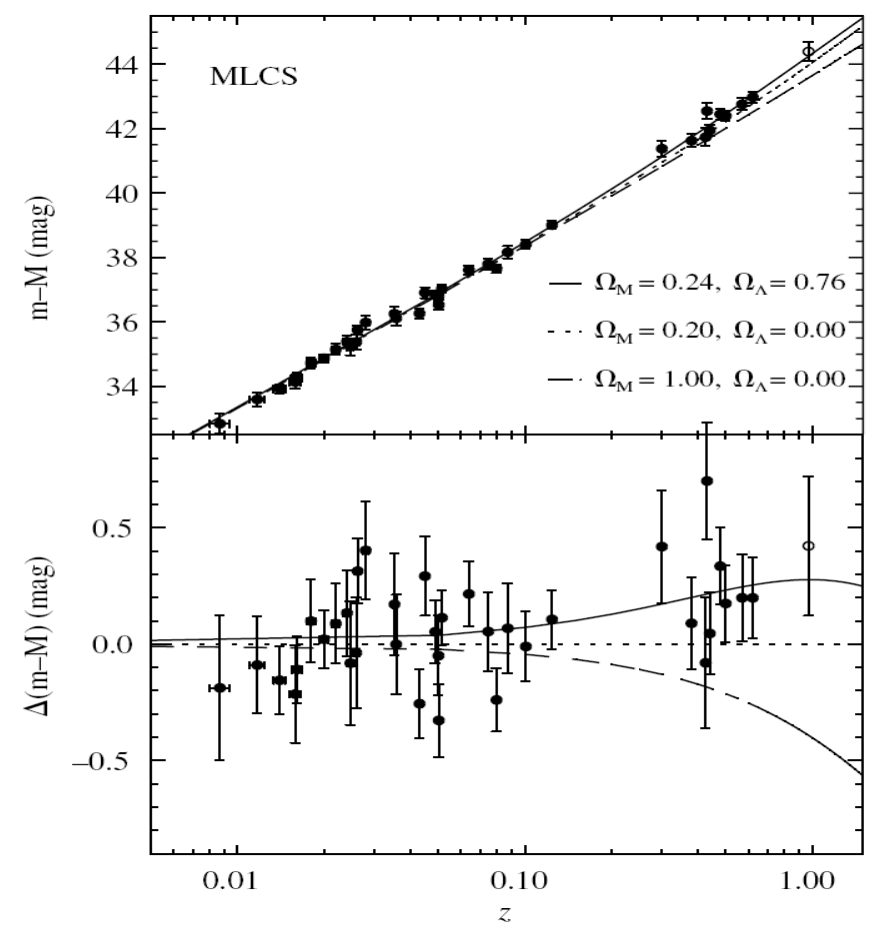

Figura 3.2: Evidência da aceleração do universo obtida com 16 supernovas em altos redshifts, incluindo duas supernovas do Supernova Cosmology Project, e 34 em baixos redshifts observadas pelo High-z Supernova Search. A figura de baixo mostra a magnitude residual de um modelo com $\Omega_{M}=0.2$ e $\Omega_{\Lambda}=0$.

\subsection{A Constante Cosmológica $\Lambda$}

A constante cosmológica, representada por $\Lambda$, é a candidata mais simples à energia escura. Ela foi proposta inicialmente por Einstein em 1917, onde ao propor um modelo cosmológico percebeu que soluções estáticas só eram possíveis através da introdução de tal constante. Na Relatividade Geral $\Lambda$ pode ser considerada um parâmetro livre, sendo a mais simples generalização da densidade Lagrangiana de Einstein-Hilbert (Weinberg, 1972).

Também podemos interpretar a constante cosmológica como pertencente ao tensor de energia-momento, onde ela passa a ser associada à energia do vácuo dos campos quânticos (Weinberg, 1989). Obtemos esse resultado ao notarmos que todos os observadores devem ver o mesmo vácuo, isto é, sua densidade de energia deve ser constante. Aplicando tal resultado para uma transformação geral de coordenadas, chegamos que o vácuo deve obedecer à equação de estado $p=-\rho$, que é a mesma obtida para a constante cosmológica 


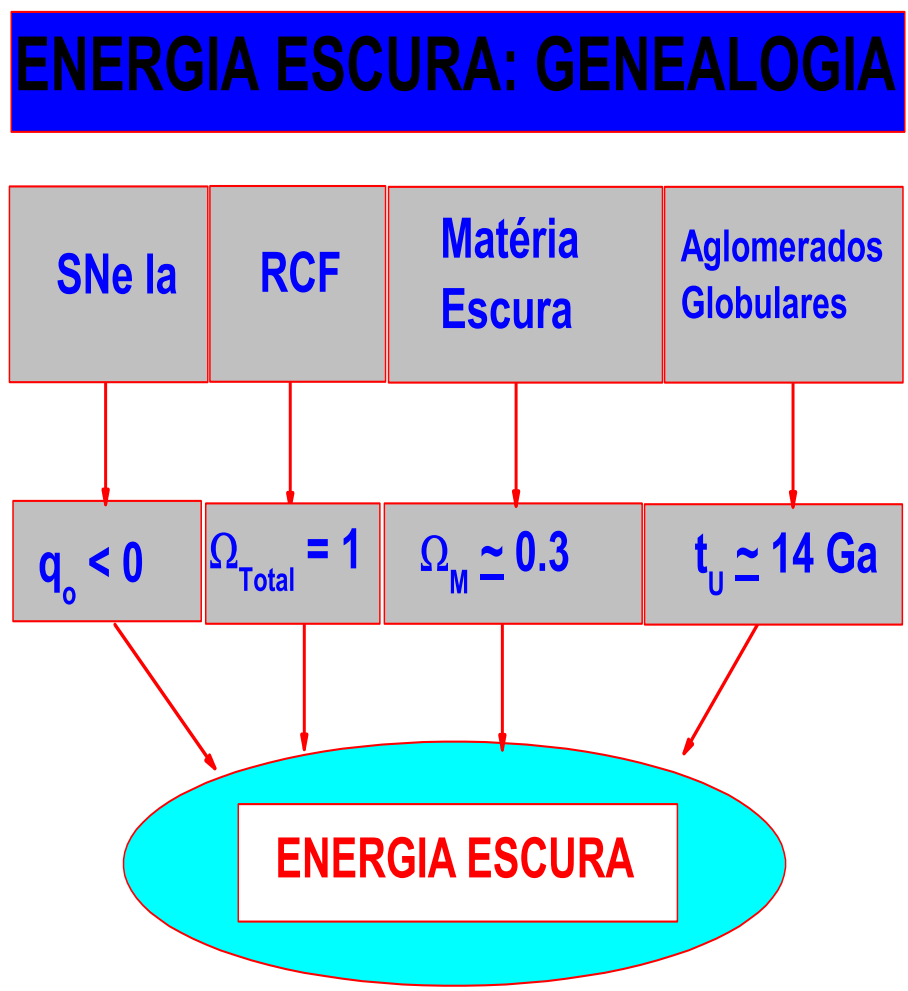

Figura 3.3: Genealogia da energia escura.

(Gron, 1986).

O espectro de potência das anisotropias de temperatura da radiação cósmica de fundo (Komatsu et al., 2008), juntamente com a determinação da constante de Hubble (Freedman et al., 2001), implicam que o universo é aproximadamente plano (dados provenientes apenas do WMAP não vinculam o parâmetro de curvatura, ver figura 3.4). Logo podemos estimar observacionalmente a contribuição da energia do vácuo, como sendo da ordem da densidade crítica hoje:

$$
\rho_{V} \approx \frac{H_{0}^{2}}{8 \pi G} \approx 10^{-47} G e V^{4}
$$

De posse do valor obtido observacionalmente, podemos comparar com o valor obtido pela teoria quântica de campos. Entendemos a energia do vácuo como a energia de ponto zero para um conjunto infinito de osciladores independentes. Para tal, integramos sobre todos os modos normais de um campo escalar de massa $m$, até um valor determinado $M$ $(\hbar=1)$ (Weinberg, 1989): 


$$
\rho_{V}=\frac{1}{4 \pi^{2}} \int_{0}^{M} K^{2} d K \sqrt{K^{2}+m^{2}} \approx \frac{1}{4 \pi^{2}} \int_{0}^{M} K^{3} d K=\frac{M^{4}}{16 \pi^{4}}
$$

O valor de $M$ é obtido a partir da hipótese de até qual escala de energia a TRG é válida. Podemos considerar por exemplo a escala de Planck, a Eletrofraca e a Cromodinâmica Quântica. Se tomarmos a cromodinâmica quântica, obtemos uma diferença de 42 ordens de grandeza entre o valor teórico e o observacional. Para a escala eletrofraca, 54 ordens de grandeza e para a escala de Planck 121 ordens de grandeza. Essa discrepância é a maior observada para uma teoria física e constitui o chamado problema da constante cosmológica. Embora existam propostas para sua solução, esse problema permanece em aberto. Apesar disso, o modelo cosmológico composto de constante cosmológica junto com matéria escura fria (CDM), conhecido como $\Lambda$ CDM, é o que melhor ajusta os dados observacionais, sendo chamado de modelo de concordância cósmica.

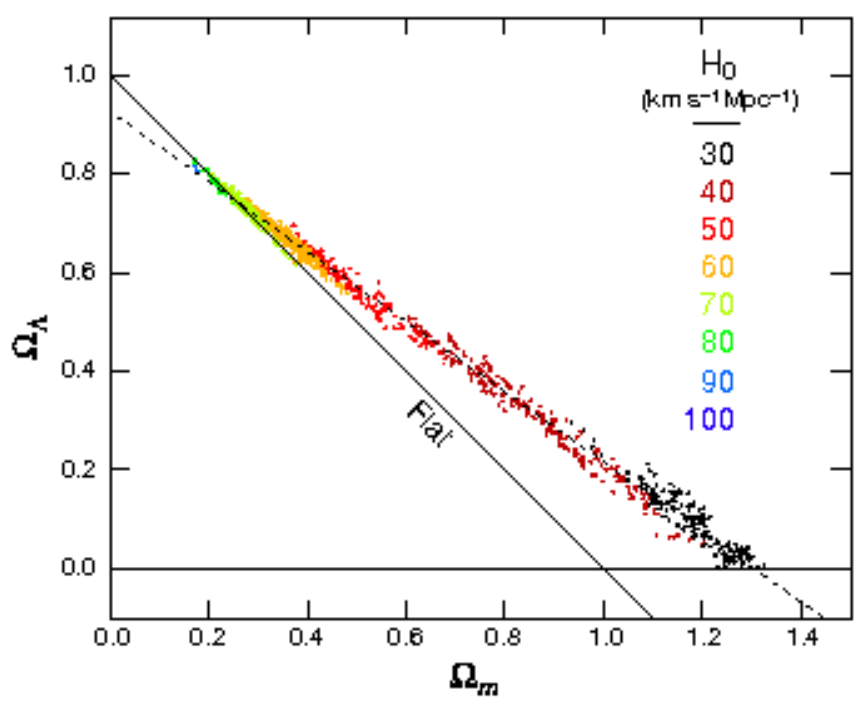

Figura 3.4: Intervalo de modelos cosmológicos não-planos consistentes com o WMAP. Resultado obtido por Spergel et al. (2007).

A análise utilizando 307 supernovas tipo Ia feita por Kowalski et al. (2008) para um modelo plano resultou em $\Omega_{M}=0.287_{-0.027}^{+0.029}(\text { stat })_{-0.036}^{+0.039}($ sis $)$, onde stat se refere ao erro estatístico e sis ao erro sistemático, com um nível de confiança estatística de 68.3\%. Combinando a análise com dados da oscilação acústica dos bárions e da radiação cósmica de fundo e deixando o parâmetro de curvatura livre eles obtiveram: $\Omega_{M}=0.285_{-0.019}^{+0.020}(\text { stat })_{-0.011}^{+0.011}(\mathrm{sis})$ e $\Omega_{k}=-0.009_{-0.010}^{+0.009}(\text { stat })_{-0.003}^{+0.002}($ sis $)$, com um nível de confiança estatística de $68.3 \%$. 


\subsection{Matéria X}

A matéria X é um fluido que obedece à equação de estado $p_{x}=w(z) \rho_{x}$, onde $w<-\frac{1}{3}$ a fim de que o universo passe por um estágio acelerado. Vemos que quando $w=-1$ retomamos o caso para uma constante cosmológica. Nesse cenário temos a matéria X e a matéria escura fria, conhecido como XCDM. Ele foi proposto por Turner e White (1997) no contexto de cosmologias aceleradas, sendo bastante discutido na literatura.

Primeiramente, descrevemos o caso onde $w$ é constante. Nesse cenário existem dois intervalos de interesse. O primeiro é o intervalo $-\frac{1}{3}<w \leq-1$, conhecido como modelo XCDM padrão, e o segundo é o intervalo $w<-1$, conhecido como modelo XCDM extendido, ou energia fantasma (phantom energy). O modelo XCDM extendido foi proposto inicialmente por Caldwell (2002). Nesse caso, pela análise da equação (1.19), vemos que a densidade de energia cresce com o tempo. Como conseqüência, temos que o universo atingirá o "Big Rip", uma singularidade em um tempo finito devido ao fato da densidade de energia divergir.

Uma análise termodinâmica para o modelo XCDM extendido foi realizada por Lima e Maia (1995a); Lima e Alcaniz (2004), enquanto para o padrão foi feita por Lima e Maia (1995b). A temperatura da matéria X cresce com o tempo: $\mathrm{Ta}^{1+w}=$ constante. Outra peculiaridade é a entropia, dada por: $S \propto(1+w) T^{3} a^{3}$, que nesse caso é negativa, em desacordo com a segunda lei da termodinâmica. Para contornar esse problema, GonzálezDíaz e Sigüenza (2004) propuseram que o fluido possui uma temperatura negativa, o que torna a entropia positiva. Mais recentemente, Lima e Pereira (2008) mostraram que se o fluido possuir um potencial químico não-nulo a temperatura e a entropia são positivas (veja também Pereira e Lima (2008)). A discussão da viabilidade teórica do modelo XCDM extendido se deve em grande parte ao fato do modelo ser compatível com as observações, constituindo um bom candidato à energia escura.

Agora, descrevemos o caso onde $w$ é uma função do redshift. Resolvendo a equação de conservação de energia (1.18) obtemos para a densidade de energia da matéria X:

$$
\rho_{X}(z)=\rho_{X 0} \exp \left(\int_{0}^{z} \frac{1+w\left(z^{\prime}\right)}{1+z^{\prime}} d z^{\prime}\right)
$$

A dependência do parâmetro $w$ com o redshift é tratada fenomenologicamente através de parametrizações. Descrevemos aqui duas possibilidades que têm sido bastante discutidas 
na literatura. A primeira é simplesmente uma expansão linear em $z$ :

$$
w(z)=w_{0}+w_{1} z
$$

onde $w_{1}=\left(\frac{d w(z)}{d z}\right)_{z=0}$. Ela foi proposta por Huterer e Turner (2001) e por Weller e Albrecht (2002), onde a densidade de energia é dada por:

$$
\rho_{X}(z)=\rho_{X 0}(1+z)^{3\left(1+w_{0}-w_{1}\right)} e^{3 w_{1} z}
$$

É importante mencionar que essa parametrização diverge para altos redshifts. A outra parametrização com grande destaque na literatura foi proposta por Chevallier e Polarski (2001) e por Linder (2003), para resolver a divergência de altos redshifts de (3.4):

$$
w(z)=w_{0}+w_{a} \frac{z}{1+z} .
$$

Neste caso, a densidade de energia é dada por:

$$
\rho_{X}(z)=\rho_{X 0}(1+z)^{3\left(1+w_{0}+w_{a}\right)} e^{-\frac{3 w_{a} z}{1+z}}
$$

onde para altos redshifts o parâmetro $w$ tende a $w(z)=w_{0}+w_{a}$. Várias parametrizações para a equação de estado da matéria X são discutidas em Johri (2004).

Um teste estatístico utilizando supernovas tipo Ia, oscilação acústica dos bárions e radiação cósmica de fundo para a equação de estado $p=w \rho$ feito por Kowalski et al. (2008) obteve o seguinte resultado: $\Omega_{M}=0.285_{-0.020}^{+0.020}(\text { stat })_{-0.010}^{+0.010}($ sis $), \Omega_{k}=-0.010_{-0.011}^{+0.010}($ stat $)$ ${ }_{-0.004}^{+0.006}(\mathrm{sis})$ e $w=-1.001_{-0.073}^{+0.069}(\mathrm{stat})_{-0.082}^{+0.080}(\mathrm{sis})$, com um nível de confiança estatística de $68.3 \%$. Partindo de um modelo plano, os resultados obtido foram: $\Omega_{M}=0.274_{-0.016}^{+0.016}$ (stat)${ }_{-0.012}^{+0.013}(\operatorname{sis})$ e $w=-0.969_{-0.063}^{+0.059}(\text { stat })_{-0.066}^{+0.063}($ sis $)$, com um nível de confiança estatística de $68.3 \%$. Já para a equação de estado (3.6) os resultados obtidos foram bem menos restringentes e estão apresentados na figura 3.5. Estudos sobre o tamanho angular mínimo de réguas padrão para esse modelo cosmológico também foram considerados (Lima e Alcaniz, 2000a,b). 


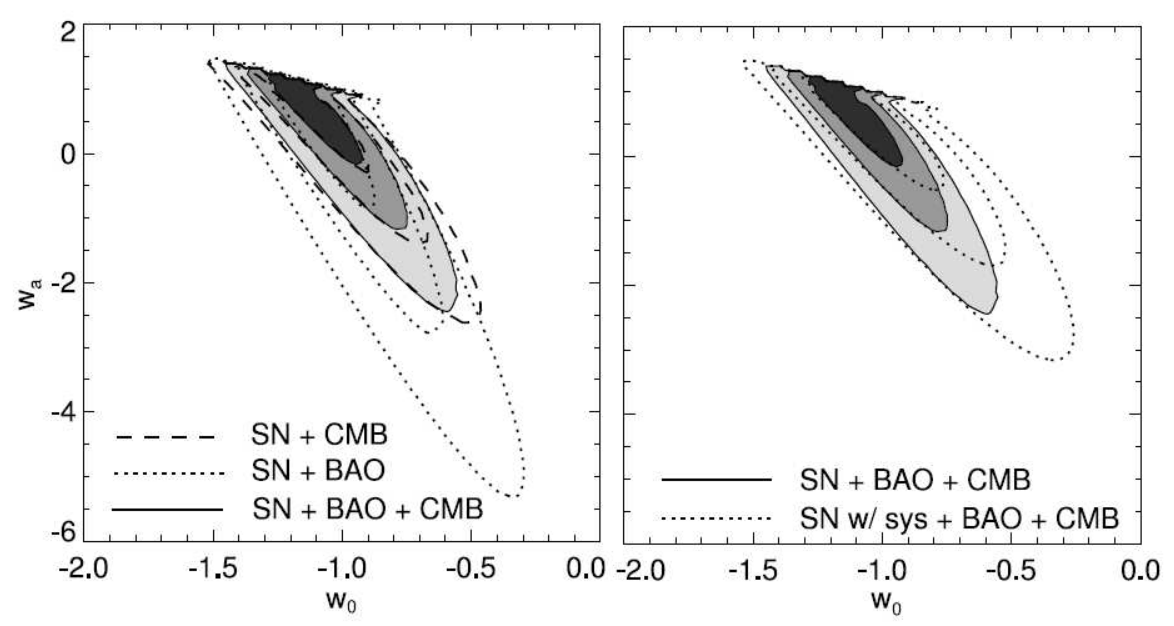

Figura 3.5: Esquerda:) O plano $w_{0}-w_{a}$ feito por Kowalski et al. (2008) para um modelo plano com equação de estado (3.6) obtido de supernovas tipo Ia (SN), oscilação acústica dos bárions (BAO) e radiação cósmica de fundo (CMB). Os contornos representam níveis de significância de $68.3 \%, 95.4 \%$ e 99.7\%. Direita:) Novamente o mesmo plano mas agora evidencia-se a diferença obtida quando os erros sistemáticos estão ou não (w /sys) inclusos na análise.

\section{$3.4 \Lambda(t)$}

Modelos onde a constante cosmológica varia com o tempo foram propostos para que fosse possível explicar a diferença entre a energia do vácuo estimada através de observações cosmológicas com a prevista pela teoria quântica de campos. Assim sendo, tais modelos assumem que a densidade de energia do vácuo decai ao longo da expansão devido ao seu acoplamento com os outros campos de matéria, até atingir o valor presentemente observado. Dessa forma, o pequeno valor atual de $\rho_{V}$ seria uma conseqüência do longo tempo de expansão do universo. Em outras palavras, $\Lambda$ é pequena porque o universo é velho. As equações de Friedmann (1.16) e (1.17) e a equação da conservação de energia (1.18) para esse modelo são dadas por:

$$
\begin{gathered}
8 \pi G \rho+\Lambda(t)=3 \frac{\dot{a}^{2}}{a^{2}}+3 \frac{k}{a^{2}}, \\
8 \pi G p-\Lambda(t)=-2 \frac{\ddot{a}}{a}-\frac{\dot{a}^{2}}{a^{2}}-\frac{k}{a^{2}},
\end{gathered}
$$




$$
\dot{\rho}+3 \frac{\dot{a}}{a}(\rho+p)=-\frac{\dot{\Lambda}}{8 \pi G},
$$

onde devemos analisar fenomenologicamente o parâmetro $\Lambda(t)$. Esse modelo foi estudado primeiramente por Bronstein (1933), mas foi só no final da década de 80, através dos trabalhos de Ozer e Taha $(1986,1987)$ que o assunto ressurgiu na literatura. Geralmente, a maioria dos artigos supõem uma dependência do parâmetro $\Lambda$ com o fator de escala, com o parâmetro de Hubble ou com ambos (Lima e Maia, 1994; Lima e Trodden, 1996; Maia e Lima, 1999; Cunha et al., 2002; Carneiro e Lima, 2005). Uma lista extensa com diversas leis de decaimento discutidas na literatura pode ser encontrada em Overduin e Cooperstock (1998). Limites oriundos da termodinâmica de não-equilíbrio para o decaimento do vácuo e seu processo de produção de partículas foram também investigados (Lima, 1996).

Outra possibilidade de analisar o decaimento do vácuo foi proposta por Wang e Meng (2005). Tal proposta é interessante pois ao invés de fornecer a dependência temporal de $\Lambda$, ela verifica quais são os efeitos na densidade de matéria escura. Tomemos novamente a equação de conservação de energia (3.10), mas agora consideremos a interação entre a matéria escura e $\Lambda(t)$ :

$$
\dot{\rho}_{M}+3 \frac{\dot{a}}{a} \rho_{M}=-\dot{\rho}_{v}
$$

onde $\rho_{M}$ é a densidade de energia da matéria escura, $\rho_{v}$ é a densidade de energia do vácuo e o ponto significa derivação com relação ao tempo. Sem o decaimento, sabemos que $\rho_{M} \propto(1+z)^{3}$. Aqui, consideremos um pequeno desvio do comportamento padrão, a saber:

$$
\rho_{M}=\rho_{M 0}(1+z)^{3-\epsilon}
$$

Utilizando as duas equações acima, obtemos para a densidade de energia do vácuo:

$$
\rho_{v}=\tilde{\rho}_{v 0}+\frac{\epsilon \rho_{M 0}}{3-\epsilon}(1+z)^{3-\epsilon},
$$

onde $\rho_{M 0}$ é a densidade de energia da matéria escura medida hoje e $\tilde{\rho}_{v 0}$ é o estado fundamental do vácuo. Duas possibilidades para o decaimento do vácuo foram analisadas por Alcaniz e Lima (2005a), a primeira sendo o decaimento do vácuo em partículas de matéria 
escura e a segunda o decaimento da energia do vácuo alteraria a massa das partículas da matéria escura. Eles testaram esse modelo, considerando o universo plano, usando dados de supernovas, radiação cósmica de fundo e aglomerados de galáxias e chegaram ao melhor ajuste dado por $\epsilon=0.06$ e $\Omega_{M}=0.27$, onde $\Omega_{M}$ é o parâmetro de densidade da matéria escura. É importante ressaltar que eles introduziram bárions neste modelo, cujo efeito foi mudar o redshift de transição e torná-lo compatível com estimativas utilizando supernovas do tipo Ia. Quando consideramos também os bárions e tomando a curvatura da secção espacial nula, o parâmetro de Hubble é dado por:

$$
H^{2}=H_{0}^{2}\left[\Omega_{b}(1+z)^{3}+\frac{3 \Omega_{M}}{3-\epsilon}(1+z)^{3-\epsilon}+\tilde{\Omega}_{v 0}\right]
$$

onde $\tilde{\Omega}_{v 0}$ é o parâmetro de densidade do estado fundamental do vácuo e $\Omega_{b}$ é o parâmetro de densidade dos bárions.

\subsection{Gás de Chaplygin}

Outro candidato para a energia escura que pode causar a expansão acelerada do universo é o gás de Chaplygin, caracterizado por uma equação de estado $p=-\frac{A}{\rho_{C g}^{\alpha}}$, onde a constante $A$ está relacionada à velocidade do som no fluido e $\alpha=1$. Quando $\alpha \neq 1$ temos o gás de Chaplygin generalizado e quando $\alpha=0$ retornamos à constante cosmológica. A vantagem de tal modelo provém do fato que este pode dar uma explicação conjunta para o problema da energia escura e para o problema da matéria escura já que os dois regimes, matéria não relativística e energia escura com pressão negativa, são contemplados para o gás de Chaplygin.

Da equação de conservação de energia (1.18) obtemos:

$$
\rho_{C g}=\rho_{C g_{0}}\left[A_{S}+\left(1-A_{S}\right)(1+z)^{3(1+\alpha)}\right]^{\frac{1}{1+\alpha}}
$$

onde $A_{S}=A / \rho_{C g_{0}}^{1+\alpha}$ e $\rho_{C g_{0}}$ é a densidade de energia do gás de Chaplygin medida hoje. Vemos que a equação acima expressa os dois comportamentos supracitados, pois para $z \rightarrow \infty$ temos $\rho \propto(1+z)^{3}$ e para $z \rightarrow 0$ temos $\rho \propto$ cte.

Para um universo dominado por um gás de Chaplygin, o parâmetro de Hubble é dado por: 


$$
H^{2}=H_{0}^{2}\left\{\Omega_{j}(1+z)^{3}+\left(1-\Omega_{j}\right)\left[A_{S}+\left(1-A_{S}\right)(1+z)^{3(\alpha+1)}\right]^{\frac{1}{\alpha+1}}\right\},
$$

onde $\Omega_{j}$ é o parâmetro de densidade que pode ser da matéria escura ou da matéria bariônica mais energia escura dependendo do cenário analisado.

Os parâmetros $\alpha$ e $A_{S}$ foram vinculados utilizando dados de supernovas e emissão em raios X de aglomerados de galáxias por Cunha et al. (2004). Eles obtiveram $A_{S}>0.84$ e e $0 \leq \alpha \leq 1$ com $68.3 \%$ de confiança estatística. Já o teste de diâmetro angular foi realizado por Alcaniz e Lima (2005b), onde obtiveram como melhor ajuste $\alpha=1$ e $A_{S}=0.99$. Tais resultados estão em desacordo com a análise da radiação cósmica de fundo com os dados do WMAP feita por Amendola et al. (2003), que exclui a região $\alpha>0.2$. Mais recentemente, um modelo com apenas um parâmetro livre foi proposto, o chamado gás de Chaplygin simplificado (Lima et al., 2006, 2008).

\subsection{Modelo Cardassiano}

O modelo cardassiano é um modelo onde o universo é plano, acelerado, dominado pela matéria escura, tendo sido proposto por Freese e Lewis (2002). Tal fato pode ser explicado se nosso universo observável estiver em uma brana tridimensional mergulhada em um universo com mais dimensões espaciais ou no caso da matéria escura apresentar auto-interação onde a força cresce com a distância (Freese e Lewis, 2002; Gondolo e Freese, 2003).

O modelo cardassiano consiste em mudar as equações de Friedmann, adicionando um termo extra na equação que dá o parâmetro de Hubble:

$$
H^{2}=\frac{8 \pi G}{3} \rho+B \rho^{n}
$$

Como temos conservação de energia e momento, obtemos novamente para a densidade de energia da matéria a equação (1.20). Podemos obter a equação acima para qualquer $n$ partindo de universos com mais dimensões (Chung e Freese, 2000). O teste de diâmetro angular para esse modelo foi realizado por Zhu e Fujimoto (2002), mas tal modelo falha quando aplicado em campos fracos (Freese, 2005), pois surge um termo a mais na equação de Poisson que destrói as curvas de rotação. Por isso, foi proposto o modelo cardassiano 
generalizado (Freese, 2003; Gondolo e Freese, 2003), onde o parâmetro de Hubble é dado por:

$$
H^{2}=\frac{8 \pi G}{3} \rho\left[1+\left(\frac{\rho}{\rho_{\text {car }}}\right)^{q(n-1)}\right]^{1 / q},
$$

onde $\rho_{\text {car }}$ corresponde ao ponto onde a densidade de energia da matéria é igual à densidade de energia do novo termo.

Os parâmetros desse modelo foram vinculados por Wang (2007) utilizando supernovas Ia, radiação cósmica de fundo, oscilação acústica dos bárions (BAO), fração de massa do gás e dados de $H(z)$, para o modelo cardassiano e cardassiano generalizado. Para o modelo cardassiano normal foram encontrados $\Omega_{M}=0.27 \pm 0.03$ e $n=0.01 \pm 0.09$ com um nível de confiança de $68.3 \%$, enquanto para o modelo generalizado foram obtidos $q=0.27_{-0.25}^{+3.65}$ e $n=-1.86_{-4.20}^{+2.26}$ com um nível de confiança de $68.3 \%$. Mosquera Cuesta et al. (2008) também vincularam os parâmetros desse modelo usando gamma-ray bursts, $\mathrm{BAO}$ e radiação cósmica de fundo. Eles obtiveram $\Omega_{M}=0.32 \pm 0.04$ e $n=0.34 \pm 0.16$ com um nível de confiança de $68.3 \%$.

\subsection{Modelo com Criação de Matéria}

Diferentemente do modelo cardassiano, este modelo é proposto no contexto da Relatividade Geral. A criação de matéria escura fria, induzida pela variação do campo gravitacional do universo, é responsável por uma pressão negativa capaz de acelerar o universo (Prigogine et al., 1989; Lima et al., 1991; Calvão et al., 1992; Abramo e Lima, 1996; Lima, 1997; Lima e Abramo, 1999; Alcaniz e Lima, 1999a).

Processos envolvendo criação de matéria pelo campo gravitacional utilizando teoria quântica de campos em espaços curvos foram estudados por Parker e colaboradores (Parker, 1968, 1969; Fulling et al., 1974; Hu e Parker, 1978; Papastamatiou e Parker, 1979). Embora rigoroso, esse método não foi completamente estudado devido a dificuldade de incorporá-lo às equações de Einstein.

Macroscopicamente, a primeira formulação auto-consistente para a criação de matéria foi feita por Prigogine e colaboradores (Prigogine et al., 1989). Posteriormente, uma formulação manifestamente covariante foi proposta por Lima e colaboradores (Lima et al., 
1991; Calvão et al., 1992; Lima et al., 1996). Eles também demonstraram que a criação de matéria pode ser estudada dentro do domínio da termodinâmica relativística fora do equilíbrio, sendo considerada macroscopicamente um processo irreversível.

O modelo aqui estudado foi proposto por Lima et al. (2008), embora um modelo parecido que não apresentava transição entre desaceleração e aceleração já tivesse sido estudado anteriormente (Lima et al., 1996).

As equações de Friedmann (1.16) e (1.17) para esse modelo são dadas por:

$$
\begin{gathered}
8 \pi G \rho=3 \frac{\dot{a}^{2}}{a^{2}}+3 \frac{k}{a^{2}}, \\
8 \pi G\left(p+p_{c}\right)=-2 \frac{\ddot{a}}{a}-\frac{\dot{a}^{2}}{a^{2}}-\frac{k}{a^{2}},
\end{gathered}
$$

onde $p_{c}$ é a pressão de criação. Nesse modelo o número de partículas não é conservado, fato que nos leva à taxa de criação de partículas:

$$
\frac{\dot{n}}{n}+3 \frac{\dot{a}}{a}=\frac{\psi}{n} \equiv \Gamma
$$

onde $n$ é a densidade numérica de partículas, $\psi$ é a taxa de criação de matéria e $\Gamma$ é a taxa de criação de partículas.

Quando consideramos que o processo de criação de matéria é adiabático, isto é, a entropia específica por partícula é constante, podemos expressar a pressão de criação por (Lima et al., 1991; Calvão et al., 1992; Lima e Germano, 1992):

$$
p_{c}=-\frac{\rho+p}{3 n H} \psi \equiv-\frac{\rho+p}{3 H} \Gamma .
$$

Vamos agora considerar um modelo plano, como sugerido pelos dados da radiação cósmica de fundo, mas preenchido apenas por matéria. Precisamos estabelecer a dependência de $\Gamma$ com os parâmetros cosmológicos. Com as hipóteses adotadas, o parâmetro de desaceleração é dado por:

$$
q=\frac{1}{2}\left[1-\frac{\Gamma}{H}\right]
$$

A existência de um redshift de transição $z_{t}$ onde $q=0$ implica que $\Gamma=H\left(z_{t}\right)$. Logo, para baixos redshifts esperamos que a taxa de criação seja proporcional à $H_{0}$, digamos: 
$\Gamma=3 \gamma H_{0}$, onde o parâmetro $\gamma$ depende do redshift de transição. Podemos fazer uma expansão em potências do parâmetro de Hubble, onde adicionamos o parâmetro $\beta$ :

$$
\Gamma=3 \gamma H_{0}+3 \beta H+\ldots
$$

O caso $\gamma=0$ foi estudado por Lima et al. (1996), enquanto o caso $\beta=0$ foi estudado por Zimdahl et al. (2001). Quando os dois parâmetros são diferentes de zero, o parâmetro de Hubble em função do redshift é dado por:

$$
H(z)=H_{0}\left[\frac{\gamma+(1-\gamma-\beta)(1+z)^{\frac{3}{2}(1-\beta)}}{1-\beta}\right] .
$$

Vínculos aos parâmetros $\gamma$ e $\beta$ foram recentemente estabelecidos por Lima et al. (2008) utilizando supernovas tipo Ia. Eles obtiveram como melhor ajuste $\gamma=0.7$ e $\beta=0.0$, sendo que $0.21 \leq \gamma \leq 0.75$ e $0 \leq \beta \leq 0.46$ com um nível de confiança estatística de $95 \%$. Mais recentemente ainda, a influência dos bárions no contexto desse modelo foi investigada por Steigman et al. (2008). 
Capítulo 4

\section{Influência das Inomogeneidades na Propagação da Luz: O Método de Dyer-Roeder}

\subsection{Introdução}

O universo é homogêneo apenas em grande escala. Logo, fótons de fontes distantes sentem a influência das inomogeneidades durante seu trajeto. Como não temos a métrica perturbada, uma possibilidade é caracterizar quantitativamente o efeito das inomogeneidades através do parâmetro de aglomeramento $\alpha$ (Zeldovich, 1964; Kristian e Sachs, 1966; Kantowski, 1969; Dyer e Roeder, 1972, 1973, 1974; Sereno et al., 2002; Demianski et al., 2003), que representa o efeito da magnificação sofrida pelo feixe de luz, onde podemos também considerar uma dependência com o redshift devido ao processo de formação de estruturas (Linder, 1988; Santos e Lima, 2008). Neste capítulo, estudamos a relação entre a distância de diâmetro angular e o redshift, que é utilizada para vincular os parâmetros cosmológicos, através da equação óptica de Sachs (1961). O objetivo final é obter a equação diferencial ZKDR (Zeldovich - Kantowski - Dyer - Roeder) para o modelo $\Lambda$ CDM e para o modelo com criação de matéria.

\subsection{A Equação Óptica de Sachs}

A chamada Equação Óptica de Sachs é uma equação diferencial que governa a evolução da área transversal de um feixe de luz ao longo de sua trajetória no espaço-tempo. Seguimos aqui a dedução feita por Peebles (1993), outra dedução pode ser encontrada no livro de Schneider e colaboradores (Schneider et al., 1992). Inicialmente, vamos considerar 
a equação do desvio geodésico para fótons (ver apêndice A):

$$
\xi_{; \beta \gamma}^{\alpha} k^{\beta} k^{\gamma}=R_{\beta \gamma \delta}^{\alpha} k^{\beta} k^{\gamma} \xi^{\delta}
$$

onde o 4 -vetor $k^{\alpha}$ satisfaz

$$
k^{\alpha}=\frac{d x^{\alpha}}{d \lambda} \quad k_{; \beta}^{\alpha}=0 \quad k^{\alpha} k_{\alpha}=0,
$$

o ponto e vírgula representa a derivada covariante ao longo do parâmetro afim $\lambda, R_{\beta \gamma \delta}^{\alpha}$ é o tensor de Riemann, $x^{\alpha}(\lambda)$ e $x^{\alpha}(\lambda)+\xi^{\alpha}(\lambda)$ são geodésicas vizinhas.

Agora, construímos uma base ortonormal de vetores ao longo da trajetória do feixe de luz (a propagação do feixe está representada na figura 4.1). Para isso, consideremos um referencial localmente lorentziano, onde a luz se propaga no eixo $\mathrm{z}$

$$
k^{\alpha}=(1,0,0,1) \quad L_{1}^{\alpha}=(0,1,0,0) \quad L_{2}^{\alpha}=(0,0,1,0) \quad \omega^{\alpha}=(1,0,0,-1),
$$

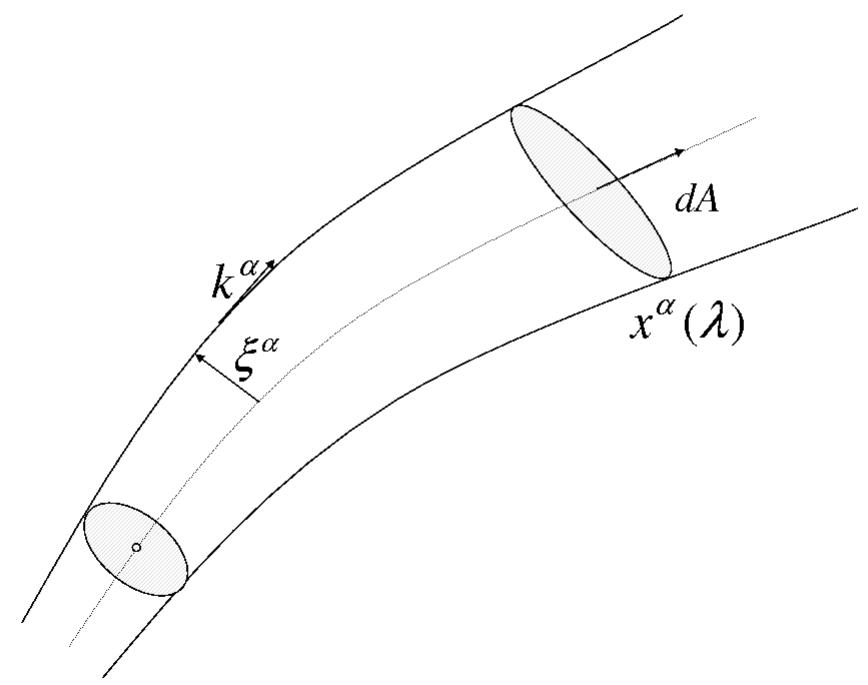

Figura 4.1: Representação da propagação de um feixe de raios de luz. As grandezas de interesse são: congruência de geodésicas nulas representadas por $x^{\alpha}(\lambda)$, elemento infinitesimal da área transversal do feixe $d A, 4$-momento dos fótons $k^{\alpha}$ e o vetor deslocamento entre duas geodésicas vizinhas $\xi^{\alpha}$. 
onde $k^{\alpha}$ é a direção de propagação da luz, $\omega^{\alpha}$ é um vetor nulo ortogonal e $L_{i}^{\alpha}$ são vetores espaciais ortogonais, onde o índice $i$ neste caso varia entre 1 e 2 . Podemos expressar as relações de ortogonalidade em um sistema de coordenadas geral da seguinte forma:

$$
g_{\alpha \beta} L_{i}^{\alpha} k^{\beta}=0 \quad g_{\alpha \beta} L_{i}^{\alpha} L_{j}^{\beta}=\delta_{i j} .
$$

É conveniente considerar um observador para o qual os vetores $L_{i}^{\alpha}$ em pontos vizinhos são paralelos ao longo do caminho do raio de luz. Tal condição pode ser expressa por

$$
L_{i ; \beta}^{\alpha} k^{\beta}=0
$$

Sabemos que o transporte paralelo dos vetores $L_{i}^{\alpha}$ e $k^{\alpha}$ os mantém normalizados e ortogonais. Isto ocorre porque ao longo da geodésica a derivada covariante de (4.4) é identicamente nula.

Vamos agora considerar raios de luz vizinhos com separação $\xi^{\alpha}(x)$ em $x$, de tal forma que um observador movendo-se livremente vê $\xi^{\alpha}$ perpendicular ao raio de luz, isto é, $k_{\alpha} \xi^{\alpha}=0$ e $\omega_{\alpha} \xi^{\alpha}=0$. As equações da geodésica asseguram que os vetores permanecem ortogonais durante a trajetória. Vamos verificar diretamente a condição $k_{\alpha} \xi^{\alpha}=0$. O 4-momento para o raio de luz é $k^{\alpha}=d x^{\alpha} / d \lambda$ em $x$ e $k^{\alpha}+d \xi^{\alpha} / d \lambda$ em $x+\xi$. A condição do segundo vetor ser do tipo nulo implica:

$$
g_{\alpha \beta}(x+\xi)\left(k^{\alpha}+\frac{d \xi^{\alpha}}{d \lambda}\right)\left(k^{\beta}+\frac{d \xi^{\beta}}{d \lambda}\right)=0 .
$$

Fazendo a expansão da equação acima e considerando apenas termos até primeira ordem em $\xi$ temos

$$
2 k^{\beta} \frac{d \xi^{\beta}}{d \lambda}+g_{\alpha \beta, \gamma} k^{\alpha} k^{\beta} \xi^{\gamma}=0
$$

Como $k_{; \beta}^{\alpha} k^{\beta}=0$, segue que

$$
\left(k_{\alpha} \xi^{\alpha}\right)_{; \beta} k^{\beta}=k_{\alpha} \xi_{; \beta}^{\alpha} k^{\beta}=k_{\alpha}\left(\frac{d \xi^{\alpha}}{d \lambda}+\Gamma_{\beta \gamma}^{\alpha} \xi^{\beta} k^{\gamma}\right)=0,
$$

onde $\Gamma_{\beta \gamma}^{\alpha}$ são os símbolos de Christoffel. Da equação acima vemos que $k_{\alpha} \xi^{\alpha}$ permanece constante ao longo da trajetória desde que essa seja a condição inicial. Como $\xi^{\alpha}$ é ortogonal a $k^{\alpha}$ e $\omega^{\alpha}$, podemos escrevê-lo como combinação linear dos $L_{i}^{\alpha}$ 


$$
\xi^{\alpha}(\lambda)=\sum_{i=1,2} D_{i}(\lambda) L_{i}^{\alpha}
$$

Substituindo em (4.1) obtemos:

$$
\frac{d^{2} D_{i}}{d \lambda^{2}}=\sum_{j} A_{i j} D_{j} \quad A_{i j}=R_{\alpha \beta \gamma \delta} L_{i}^{\alpha} k^{\beta} k^{\gamma} L_{j}^{\delta}
$$

Podemos escrever $A_{i j}$ em função do tensor de Weyl $C_{\alpha \beta \gamma \delta}$ e do tensor de Ricci $R_{\alpha \beta}$

$$
A_{i j}=-\frac{1}{2} R_{\beta \gamma} k^{\beta} k^{\gamma}+C_{\alpha \beta \gamma \delta} L_{i}^{\alpha} k^{\beta} k^{\gamma} L_{j}^{\delta}
$$

Também podemos escrever:

$$
\frac{d D_{i}}{d \lambda}=\sum_{j}\left(\theta \delta_{i j}+\sigma_{i j}\right) D_{j}
$$

onde $\theta$ é o parâmetro de expansão do feixe de raios de luz, $\sigma_{i j}$ é o tensor de cisalhamento (os efeitos destes parâmetros estão apresentados na figura 4.2), simétrico e com traço nulo, que podemos representar da seguinte maneira:

$$
\sigma_{i j}=\left(\begin{array}{cc}
\rho & \omega \\
\omega & -\rho
\end{array}\right)
$$

$\theta, \rho$ e $\sigma$ são as três funções independentes necessárias para as componentes do tensor simétrico $A_{i j}$. O tensor de cisalhamento satisfaz

$$
\sigma_{i j} \sigma_{j k}=\left(\begin{array}{cc}
\rho & \omega \\
\omega & -\rho
\end{array}\right)\left(\begin{array}{cc}
\rho & \omega \\
\omega & -\rho
\end{array}\right)=\left(\rho^{2}+\omega^{2}\right) \delta_{i k} \quad \sigma^{2}=\rho^{2}+\omega^{2} .
$$

Derivando (4.12) com relação a $\lambda$

$$
\frac{d^{2} D_{i}}{d \lambda^{2}}=\sum_{j}\left[\left(\theta^{2}+\frac{\sigma^{2}}{2}+\frac{d \theta}{d \lambda}\right) \delta_{i j}+\left(\frac{d \sigma_{i j}}{d \lambda}+2 \theta \sigma_{i j}\right)\right] D_{j} .
$$

Logo, $A_{i j}$ pode ser escrito como:

$$
A_{i j}=\left(\frac{d \theta}{d \lambda}+\theta^{2}+\frac{\sigma^{2}}{2}\right) \delta_{i j}+\left(\frac{d \sigma_{i j}}{d \lambda}+2 \theta \sigma_{i j}\right)
$$



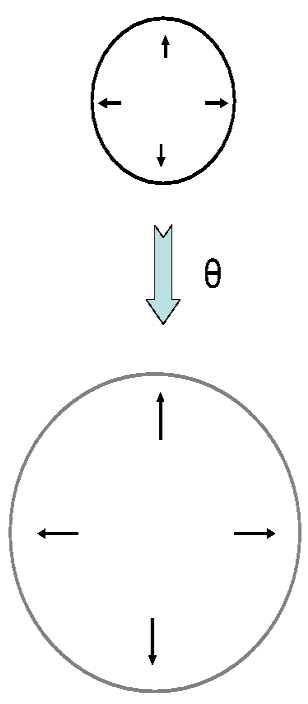

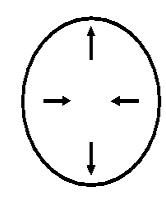

$\sigma_{\mathrm{ab}} \sqrt{\mathrm{V}}$

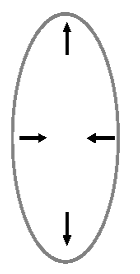

Figura 4.2: Esquerda: Ação do parâmetro de expansão $\theta$ na área transversal de um feixe de luz. Seu efeito consiste em mapear um círculo em outro círculo de maior área. Direita: Ação do tensor de cisalhamento $\sigma_{a b}$. Seu efeito na área transversal de um feixe de luz é mapear um círculo numa elipse preservando a área.

Como a seguinte equação é válida

$$
\sum_{i=1,2} C_{\alpha \beta \gamma \delta} L_{i}^{\alpha} k^{\beta} k^{\gamma} L_{j}^{\delta}=0
$$

chegamos a

$$
\theta^{2}+\frac{\sigma^{2}}{2}+\frac{d \theta}{d \lambda}=-R_{\alpha \beta} k^{\alpha} k^{\beta} \quad \frac{d \sigma_{i j}}{d \lambda}+2 \theta \sigma_{i j}=C_{\alpha \beta \gamma \delta} L_{i}^{\alpha} k^{\beta} k^{\gamma} L_{j}^{\delta} .
$$

Para vermos como a área do feixe varia ao longo da trajetória, usando (4.12)

$$
\tilde{D}_{i} \equiv D_{i}(\lambda+\delta \lambda)=D_{i}(\lambda)+\left(\theta D_{i}+\sum_{j} \sigma_{i j} D_{j}\right) \delta \lambda .
$$

A área própria da secção transversal é dada pela integral de $D_{i}$

$$
A(\lambda+\delta \lambda)=\int d \tilde{D}_{1} d \tilde{D}_{2}=\int d D_{1} d D_{2} \frac{\partial \tilde{D}}{\partial D}=\frac{\partial \tilde{D}}{\partial D} A(\lambda)
$$

e calculando o jacobiano acima temos: 


$$
\frac{A(\lambda+\delta \lambda)}{A(\lambda)}=\left|\begin{array}{cc}
1+\left(\theta+\sigma_{11}\right) \delta \lambda & \sigma_{12} \delta \lambda \\
\sigma_{21} \delta \lambda & 1+\left(\theta+\sigma_{11}\right) \delta \lambda
\end{array}\right|=1+2 \theta \delta \lambda
$$

tomando o limite $\delta \lambda \rightarrow 0$ obtemos:

$$
A(\lambda+\delta \lambda)=A(\lambda)+2 \theta \delta \lambda A(\lambda) \Rightarrow \frac{d A}{d \lambda}=2 \theta A
$$

Com as expressões acima podemos escrever

$$
\frac{1}{A} \frac{d^{2} A}{d \lambda^{2}}=\frac{1}{2}\left(\frac{1}{A} \frac{d A}{d \lambda}\right)^{2}-\sigma^{2}-R_{\alpha \beta} k^{\alpha} k^{\beta},
$$

que pode ser reescrita na forma conhecida como equação óptica de Sachs:

$$
\frac{1}{\sqrt{A}} \frac{d^{2} \sqrt{A}}{d \lambda^{2}}=-\frac{1}{2}\left(\sigma^{2}+R_{\alpha \beta} k^{\alpha} k^{\beta}\right)
$$

\subsection{Equação ZKDR para o modelo $\Lambda C D M$}

Deduzimos aqui a equação diferencial ZKDR (Zeldovich-Kantowski-Dyer-Roeder) para o modelo $\Lambda$ CDM. Consideramos o universo plano sendo composto por matéria escura e constante cosmológica $\Lambda$. Logo, o tensor de energia e momento é dado por:

$$
T^{\mu \nu}=\rho_{M} u^{\mu} u^{\nu}+\rho_{\Lambda} g^{\mu \nu},
$$

onde $u^{\mu}=\delta_{0}^{\mu}$ é a quadrivelocidade dos elementos de volume comóveis. As equações de Einstein são dadas por:

$$
G^{\mu \nu} \equiv R^{\mu \nu}-\frac{1}{2} R g^{\mu \nu}=8 \pi G T^{\mu \nu}
$$

onde $G^{\mu \nu}$ é o tensor de Einstein e R é o escalar de Ricci. Agora, utilizaremos a equação de Sachs com tensor de cisalhamento nulo, uma vez que estamos lidando com o universo aproximadamente homogêneo. Para um universo conformalmente plano como o FLRW, é fácil mostrar que $C_{\alpha \beta \gamma \delta}=0$ (Weinberg, 1972). Logo, se o tensor de cisalhamento for inicialmente nulo, durante a propagação tal quantidade manterá esse valor (Schneider et al., 1992). Como a distância de diâmetro angular é proporcional à raiz da área, usamos $D_{A}$. Assim, temos: 


$$
\frac{d^{2} D_{A}}{d \lambda^{2}}+\frac{1}{2} D_{A} 8 \pi G T_{\mu \nu} k^{\mu} k^{\nu}=0
$$

É importante relacionar o parâmetro afim $\lambda$ com alguma quantidade observável. O redshift z é a grandeza mais conveniente para parametrizar a trajetória dos fótons. A freqüência de um fóton medida por um observador comóvel é dada por (Schneider et al., 1992):

$$
\omega=u^{\alpha} k_{\alpha}=\frac{1}{H_{0}}(1+z) .
$$

A razão entre freqüências medidas por dois observadores está relacionada ao redshift por

$$
1+z=\frac{\omega}{\omega_{0}}
$$

onde $\omega_{0}$ é a freqüência medida por um observador arbitrário. Derivando a equação acima com respeito ao parâmetro afim $\lambda$

$$
\frac{d z}{d \lambda}=\frac{1}{\omega_{0}} k^{\alpha} k^{\beta} u_{\alpha ; \beta}=(1+z)^{2} H(z),
$$

podemos expressar a equação (4.27) em função do redshift

$$
\left(\frac{d z}{d \lambda}\right)^{2} \frac{d^{2} D_{A}}{d z^{2}}+\left(\frac{d^{2} z}{d \lambda^{2}}\right) \frac{d D_{A}}{d z}+4 \pi G T_{\mu \nu} k^{\mu} k^{\nu} D_{A}=0 .
$$

O modelo de Dyer-Roeder (Dyer e Roeder, 1972, 1973) consiste em assumir que as inomogeneidades são representadas por aglomerados que estão aleatoriamente distribuídos, sendo que uma parte homogeneamente distribuída com densidade menor compensa as regiões aglomeradas. Tal fato implica que em média a densidade é equivalente a um modelo FLRW, isto é, neste modelo a taxa de expansão $H(z)$ é a mesma de um modelo FLRW (para uma discussão sobre essa hipótese veja Bildhauer e Futamase (1991) e Buchert e Ehlers (1997)).

As observações realizadas geralmente não sofrem lenteamento, o que indica que a luz se propaga pela parte homogênea. Logo, ao invés invés de perceber uma densidade de energia $\rho_{M}$, a luz sentirá uma densidade efetiva $\alpha \rho_{M}$. Note que a densidade de energia do 
vácuo $\left(\frac{\Lambda}{8 \pi G}\right)$ não se aglomera. O parâmetro $\alpha$ é chamado de parâmetro de aglomeramento. Desta forma, sua definição é dada por:

$$
\alpha=\frac{\rho_{H o m}}{\rho_{M}}=\frac{\rho_{M}-\rho_{A g l}}{\rho_{M}}=1-\frac{\rho_{A g l}}{\rho_{M}} .
$$

onde $\rho_{\text {Hom }}$ é a fração da densidade de energia da matéria homogeneamente distribuída e $\rho_{\text {Agl }}$ a fração aglomerada. Vemos assim que o parâmetro $\alpha$ quantifica o total de matéria homogeneamente distribuída que o feixe de luz encontra em sua trajetória. Para $\alpha=1$ (filled beam, na literatura inglesa) toda a matéria está distribuída de forma homogênea. Para $\alpha=0$ temos um caso extremo onde toda a matéria está aglomerada (empty beam, novamente na literatura inglesa). Portanto, vemos que o parâmetro de aglomeramento para um aglomeramento parcial está no intervalo: $0<\alpha<1$.

Devido ao processo de formação de estruturas, uma dependência do parâmetro de aglomeramento com o redshift é naturalmente esperada. Tal fato foi estudado primeiramente por Linder (1988). Mais recentemente, Santos e Lima (2008) propuseram uma função para $\alpha$ dada por

$$
\alpha(z)=\frac{\beta_{0}(1+z)^{3 \gamma}}{1+\beta_{0}(1+z)^{3 \gamma}},
$$

onde $\beta_{0}$ e $\gamma$ são constantes. Essa fórmula possui as propriedades desejadas para $\alpha$. Para $z \rightarrow \infty, \alpha \rightarrow 1$, visto que antes do processo de formação de estruturas o universo era homogêneo em todas as escalas. Para $z=0, \alpha=\frac{\beta_{0}}{1+\beta_{0}}$, que é um valor intermediário entre 0 e 1. Nesta dissertação, tratamos apenas o caso onde $\alpha$ é constante.

Para o modelo $\Lambda$ CDM plano, o parâmetro de Hubble pode ser expresso como

$$
H(z)^{2}=H_{0}^{2}\left[\Omega_{M}(1+z)^{3}+\left(1-\Omega_{M}\right)\right] .
$$

Substituindo a fórmula de $H(z)$ (4.34) na equação (4.30) e utilizando a equação (4.25) podemos reescrever a equação (4.31) na forma

$$
(1+z)^{2} F \frac{d^{2} D_{A}}{d z^{2}}+(1+z) G \frac{d D_{A}}{d z}+H D_{A}=0
$$

onde

$$
F=\Omega_{M}(1+z)^{3}+\left(1-\Omega_{M}\right)
$$




$$
\begin{gathered}
G=\frac{7}{2} \Omega_{M}(1+z)^{3}+2\left(1-\Omega_{M}\right) \\
H=\frac{3 \alpha}{2} \Omega_{M}(1+z)^{3} .
\end{gathered}
$$

A equação (4.35) é conhecida como equação ZKDR e satisfaz as seguintes condições iniciais:

$$
D_{A}(0)=0 \quad\left(\frac{d D_{A}}{d z}\right)_{0}=H_{0}^{-1} .
$$

A equação (4.35) foi obtida por Alcaniz et al. (2004). Uma expressão levando em consideração o termo de curvatura foi proposta por Demianski et al. (2003). Estudos de time delays utilizando esse formalismo também foram realizados (Giovi e Amendola, 2001; Lewis e Ibata, 2002).

O teste de diâmetro angular para o modelo com matéria $\mathrm{X}$ usando fontes de rádio ultracompactas (Gurvits et al., 1999) foi realizado por Santos (2007) e Santos e Lima (2008), mas os dados não vincularam o parâmetro de aglomeramento $\alpha$.

A equação ZKDR também pode ser escrita em termos da distância de luminosidade. Para tal basta usarmos a relação (2.16), que resulta em

$$
(1+z)^{2} \mathcal{F} \frac{d^{2} D_{L}}{d z^{2}}-(1+z) \mathcal{G} \frac{d D_{L}}{d z}+\mathcal{H} D_{L}=0
$$

onde

$$
\begin{gathered}
\mathcal{F}=\Omega_{M}(1+z)^{3}+\left(1-\Omega_{M}\right)(1+z)^{-3}, \\
\mathcal{G}=\frac{1}{2} \Omega_{M}+2\left(1-\Omega_{M}\right)(1+z)^{-3}, \\
\mathcal{H}=\frac{3 \alpha-2}{2} \Omega_{M}+2\left(1-\Omega_{M}\right)(1+z)^{-3} .
\end{gathered}
$$

A equação (4.38) satisfaz as seguintes condições iniciais:

$$
D_{L}(0)=0 \quad\left(\frac{d D_{L}}{d z}\right)_{0}=H_{0}^{-1},
$$


e foi proposta por Sereno et al. (2002).

Na figura 4.3, mostramos a influência do parâmetro de aglomeramento $\alpha$ na distância de luminosidade para um valor fixo de $\Omega_{M}=0.27$. Vemos que quanto menor $\alpha$, menor é o foco do feixe. Um foco menor implica em um fluxo medido menor, logo a distância inferida é maior. Apresentamos os vínculos obtidos aos parâmetros $\alpha$ e $\Omega_{M}$ no capítulo 5 .

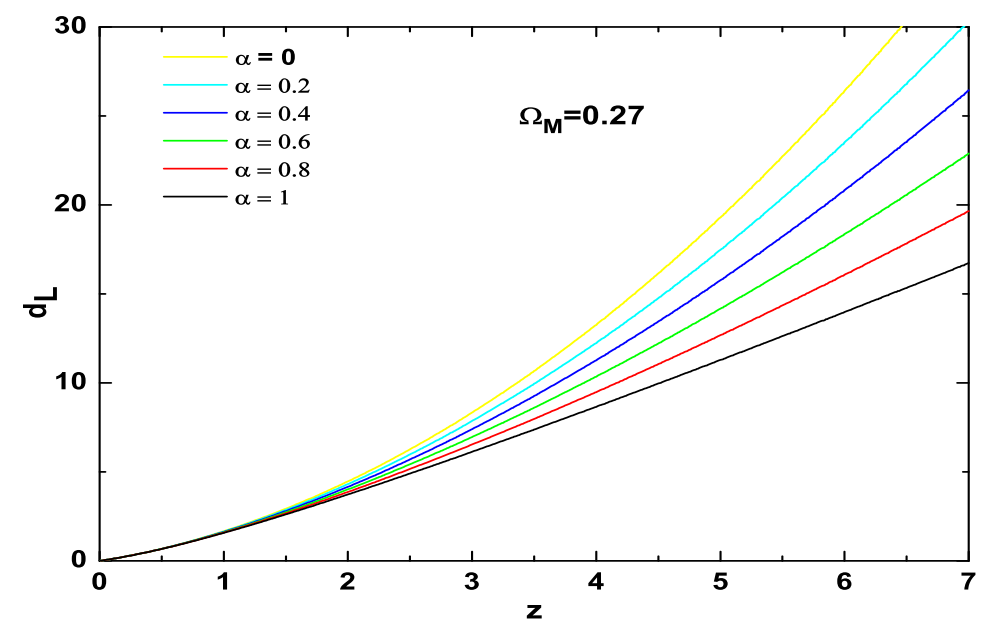

Figura 4.3: Distância de luminosidade em unidades de $c / H_{0}$ em função do redshift para vários valores do parâmetro de aglomeramento $\alpha, \operatorname{com} \Omega_{M}=0.27$.

\subsection{Equação ZKDR para o modelo com criação de matéria}

A dedução da equação ZKDR para o modelo com criação de matéria segue em linhas gerais a dedução para o modelo $\Lambda$ CDM. Vale destacar que as mudanças ocorrem no parâmetro de Hubble e no tensor de energia-momento. O parâmetro de Hubble nesse caso é dado por:

$$
H(z)=H_{0}\left[\frac{\gamma+(1-\gamma-\beta)(1+z)^{\frac{3}{2}(1-\beta)}}{1-\beta}\right] .
$$

O tensor de energia-momento para esse modelo não é descrito por um fluido perfeito, uma vez que a criação de matéria é um processo irreversível que gera uma pressão de criação. Logo, tal quantidade é expressa por (Lima et al., 2008):

$$
T^{\mu \nu}=\left(\rho+p+p_{c}\right) u^{\mu} u^{\nu}-\left(p+p_{c}\right) g^{\mu \nu},
$$


onde $p_{c}$ é a pressão de criação dada pela equação (3.22). Como neste caso o universo é composto somente por matéria escura, $p=0$. Aplicando o mesmo procedimento que foi feito para o modelo $\Lambda$ CDM, chegamos a seguinte equação ZKDR para o modelo com criação de matéria:

$$
(1+z)^{2} F \frac{d^{2} D_{A}}{d z^{2}}+(1+z) G \frac{d D_{A}}{d z}+H D_{A}=0
$$

onde

$$
\begin{gathered}
F=\frac{\gamma+(1-\gamma-\beta)(1+z)^{\frac{3}{2}(1-\beta)}}{1-\beta}, \\
G=\frac{2 \gamma+\frac{1}{2}(7-3 \beta)(1-\gamma-\beta)(1+z)^{\frac{3}{2}(1-\beta)}}{1-\beta}, \\
H=\frac{3 \alpha}{2}(1-\gamma-\beta)(1+z)^{\frac{3}{2}(1-\beta)} .
\end{gathered}
$$

As mesmas condições iniciais são satisfeitas

$$
D_{A}(0)=0 \quad\left(\frac{d D_{A}}{d z}\right)_{0}=H_{0}^{-1} .
$$

Pela utilização da relação (2.16), obtemos a equação ZKDR em função da distância de luminosidade para o modelo com criação de matéria

$$
\mathcal{F} \frac{d^{2} D_{L}}{d z^{2}}-\frac{1}{1+z} \frac{1}{1-\beta} \mathcal{G} \frac{d D_{L}}{d z}+\frac{1}{(1+z)^{2}} \mathcal{H} D_{L}=0
$$

que satisfaz as seguintes condições iniciais:

$$
\left\{\begin{array}{c}
D_{L}(0)=0, \\
\left(\frac{d D_{L}}{d z}\right)_{0}=H_{0}^{-1} .
\end{array}\right.
$$

onde $\mathcal{F}, \mathcal{G}$ e $\mathcal{H}$ são funções dos parâmetros cosmológicos, expressos em termos do redshift por: 


$$
\begin{aligned}
\mathcal{F} & =\frac{\gamma+(1-\gamma-\beta)(1+z)^{\frac{3}{2}(1-\beta)}}{1-\beta} \\
\mathcal{G} & =2 \gamma+\frac{1}{2}(1+3 \beta)(1-\gamma-\beta)(1+z)^{\frac{3}{2}(1-\beta)} \\
\mathcal{H} & =\frac{2 \gamma-(1-3 \beta)(1-\gamma-\beta)(1+z)^{\frac{3}{2}(1-\beta)}}{1-\beta}+\frac{3}{2} \alpha(1-\gamma-\beta)(1+z)^{\frac{3}{2}(1-\beta)} .
\end{aligned}
$$

Na figura 4.4, mostramos a influência do parâmetro de aglomeramento $\alpha$ sobre a distância de luminosidade para um valor fixo de $\gamma=0.6$. Vemos, de forma análoga ao ocorrido para o modelo $\Lambda$ CDM, que quanto menor $\alpha$ menor é o foco do feixe. Tal resultado implica que o fluxo medido também será menor. Portanto, a distância inferida será maior. Os vínculos obtidos aos parâmetros $\alpha$ e $\gamma$ serão apresentados no capítulo 6 .

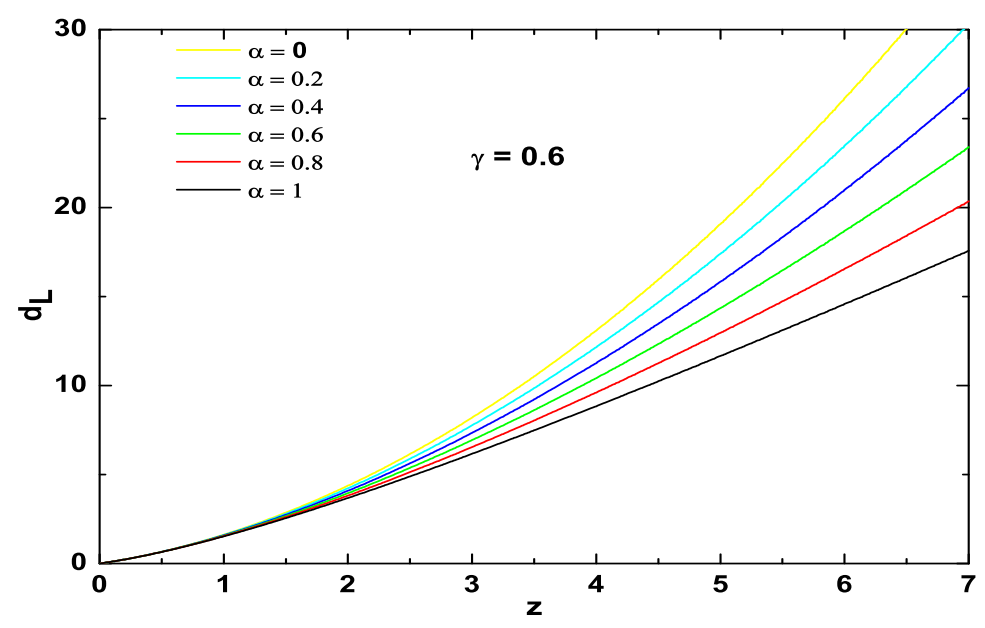

Figura 4.4: Distância de luminosidade em unidades de $c / H_{0}$ em função do redshift para vários valores do parâmetro de aglomeramento $\alpha, \operatorname{com} \gamma=0.6$. 
Capítulo 5

\section{Vínculos Observacionais para $\alpha$ e $\Omega_{M}$}

\subsection{Introdução}

Neste capítulo analisaremos os vínculos obtidos sobre o parâmetro de aglomeramento $\alpha$ e sobre o parâmetro de densidade da matéria $\Omega_{M}$ utilizando o teste $\chi^{2}$ para a amostra de supernovas do Union Compilation Data (Kowalski et al., 2008) e para os dados de GRBs discutidos no capítulo 2 para a calibração de Schaefer (2007) e para o modelo cardassiano (Mosquera Cuesta et al., 2008).

Para o modelo $\Lambda$ CDM plano, o parâmetro de Hubble pode ser escrito como

$$
H(z)=H_{0}\left[\Omega_{M}(1+z)^{3}+\left(1-\Omega_{M}\right)\right]^{\frac{1}{2}} .
$$

Neste caso, a equação ZKDR para a distância de luminosidade tem a seguinte forma

$$
(1+z)^{2} \mathcal{F} \frac{d^{2} D_{L}}{d z^{2}}-(1+z) \mathcal{G} \frac{d D_{L}}{d z}+\mathcal{H} D_{L}=0
$$

onde

$$
\begin{gathered}
\mathcal{F}=\Omega_{M}(1+z)^{3}+\left(1-\Omega_{M}\right)(1+z)^{-3}, \\
\mathcal{G}=\frac{1}{2} \Omega_{M}+2\left(1-\Omega_{M}\right)(1+z)^{-3}, \\
\mathcal{H}=\frac{3 \alpha-2}{2} \Omega_{M}+2\left(1-\Omega_{M}\right)(1+z)^{-3} .
\end{gathered}
$$

Relembramos também que o módulo de distância em função da distância de luminosidade pode ser expresso como (Peebles, 1993): 


$$
\mu=5 \log \left(D_{L}\right)+25
$$

onde $D_{L}$ é expressa em Mpc.

O $\chi^{2}$ usual é dado por:

$$
\chi^{2}=\sum_{i}^{N} \frac{\left(\mu_{\text {teor }}\left(z_{i}, \alpha, \Omega_{M}, H_{0}\right)-\mu_{i}\left(z_{i}, a_{j}, b_{j}\right)\right)^{2}}{\sigma_{\mu_{i}}\left(a_{j}, b_{j}\right)^{2}},
$$

onde $\mu_{\text {teor }}$ é o módulo de distância teórico calculado a partir de (5.2) e (5.4), $\mu_{i}$ é o módulo de distância obtido a partir dos dados observacionais, $\sigma_{\mu_{i}}$ é a incerteza do módulo de distância e $N$ é o número de dados. O teste $\chi^{2}$ consiste em determinar qual o conjunto de parâmetros que minimiza a expressão acima. Como é bem conhecido, quando estamos interessados em apenas alguns parâmetros envolvidos, temos que marginalizar sobre os outros para que não influam na análise. Devemos marginalizar sobre os parâmetros $a_{j}$ e $b_{j}$ obtidos das calibrações descritas no capítulo 2, mas como dependem fracamente da cosmologia adotada, é consistente adotar o melhor ajuste das relações. Tal hipótese é testada pela comparação dos resultados obtidos para as duas calibrações, que são discutidas neste capítulo. Também devemos marginalizar sobre $H_{0}$, onde integramos a Likelihood em $H_{0}$ (Trotta, 2008):

$$
\tilde{\mathcal{L}}=\int e^{-\frac{\chi^{2}}{2}} \pi\left(H_{0}\right) d H_{0}
$$

onde $\pi\left(H_{0}\right)$ é o prior adotado. Consideramos aqui o prior gaussiano (uma discussão da influência do prior é feita no apêndice B)

$$
\pi\left(H_{0}\right)=e^{-\frac{1}{2} \frac{\left(H_{0}-72\right)^{2}}{8^{2}}},
$$

onde $H_{0}$ é dado em $\mathrm{Km} \mathrm{s}^{-1} \mathrm{Mpc}^{-1}$. Considerar um prior gaussiano em $H_{0}$ significa que o valor real de $H_{0}$ está distribuído de forma gaussiana em torno do valor fornecido por Freedman et al. (2001), dado por $72 \mathrm{Km} \mathrm{s}^{-1} \mathrm{Mpc}^{-1}$ com um erro de $8 \mathrm{Km} \mathrm{s}^{-1} \mathrm{Mpc}^{-1}$. Logo, o novo $\chi^{2}$ é dado por:

$$
\tilde{\chi}^{2}=-2 \ln \tilde{\mathcal{L}}
$$


Na figura 5.1 mostramos a influência do parâmetro de aglomeramento $\alpha$ comparando modelos com diferentes valores de $\alpha$ a um modelo vazio, tanto para os dados de supernovas quanto para os dados de GRBs utilizando as duas calibrações.
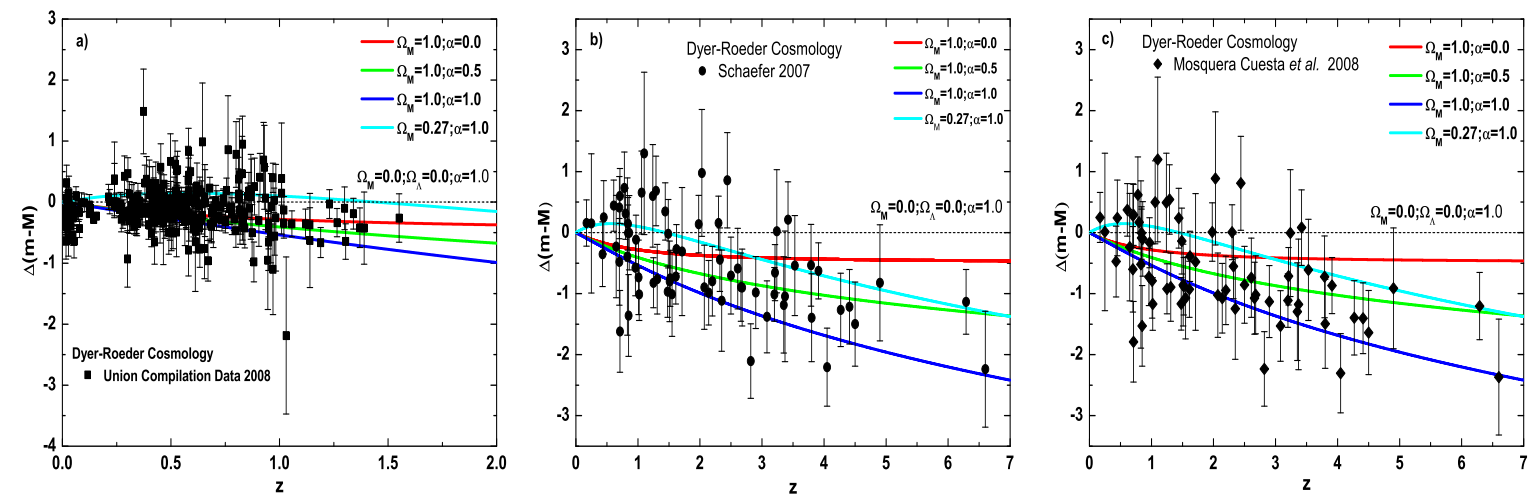

Figura 5.1: Magnitude residual comparada a um universo vazio homogêneo e isotrópico em todas as escalas para: a) 307 supernovas b) 69 GRBs calibrados por Schaefer c) 69 GRBs calibrados para o modelo cardassiano.

\subsection{Supernovas}

Obtivemos vínculos aos parâmetros $\alpha$ e $\Omega_{M}$ através do teste $\chi^{2}$ utilizando 307 supernovas da amostra de Kowalski et al. (2008) (Union Compilation Data). Os resultados obtidos são mostrados na figura 5.2. Vemos que o parâmetro de densidade da matéria $\Omega_{M}$ foi bem vinculado, diferentemente do que ocorreu com o parâmetro de aglomeramento $\alpha$. O melhor ajuste para $\Omega_{M}$ é consistente com testes independentes, enquanto o melhor ajuste para $\alpha$ privilegia um universo homogêneo e isotrópico em todas as escalas.

\subsection{Gamma-Ray Bursts}

Realizamos o teste $\chi^{2}$ para a amostra de 69 gamma-ray bursts calibrados por Schaefer (2007) a fim de vincular os parâmetros $\alpha$ e $\Omega_{M}$. Os vínculos obtidos estão apresentados na figura 5.3. Notamos que não houve melhora significativa no vínculo em $\alpha$, sendo o parâmetro $\Omega_{M}$ fracamente vinculado. Vemos que altos valores de $\Omega_{M}$ são permitidos. Este fato pode ser compreendido uma vez que dados em altos redshifts não vinculam a energia escura. 

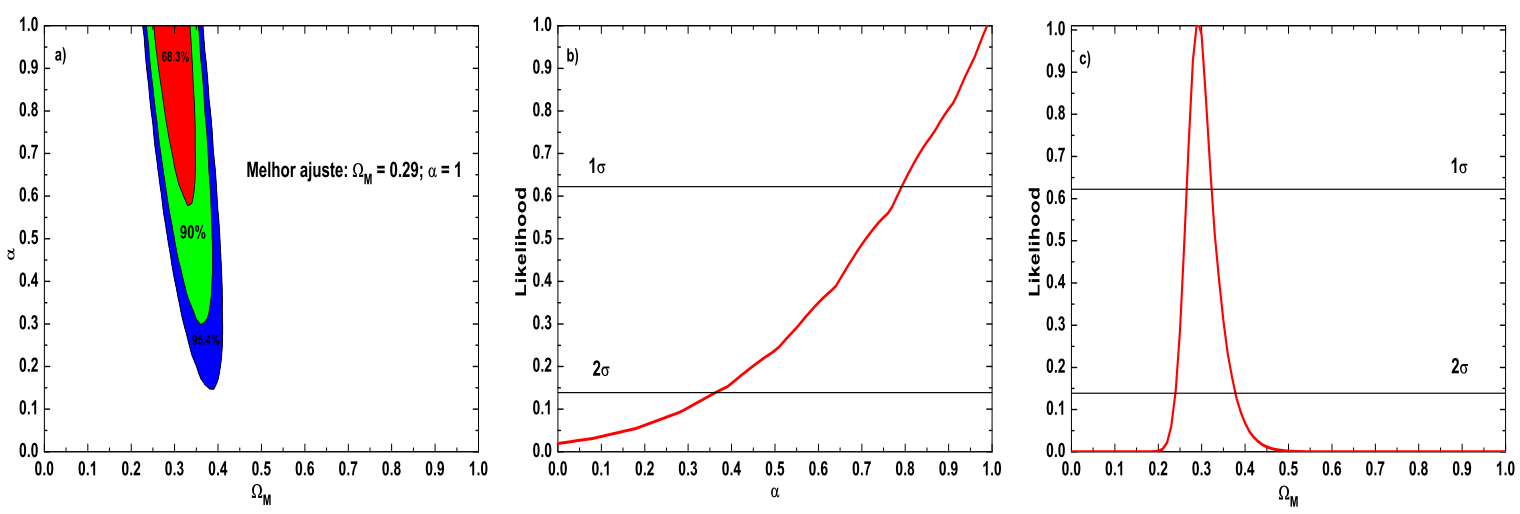

Figura 5.2: a) Plano $\Omega_{M}-\alpha$ para o modelo $\Lambda$ CDM plano obtido de 307 dados de supernovas. Os níveis de significância são os indicados na figura. b) Likelihood para o parâmetro de aglomeramento $\alpha$. Com um nível de significância de $95.4 \%$ temos que $0.36 \leq \alpha \leq 1.0$. c) Likelihood para o parâmetro de densidade da matéria $\Omega_{M}$. Com um nível de significância de $95.4 \%$ temos que $0.24 \leq \Omega_{M} \leq 0.37$.
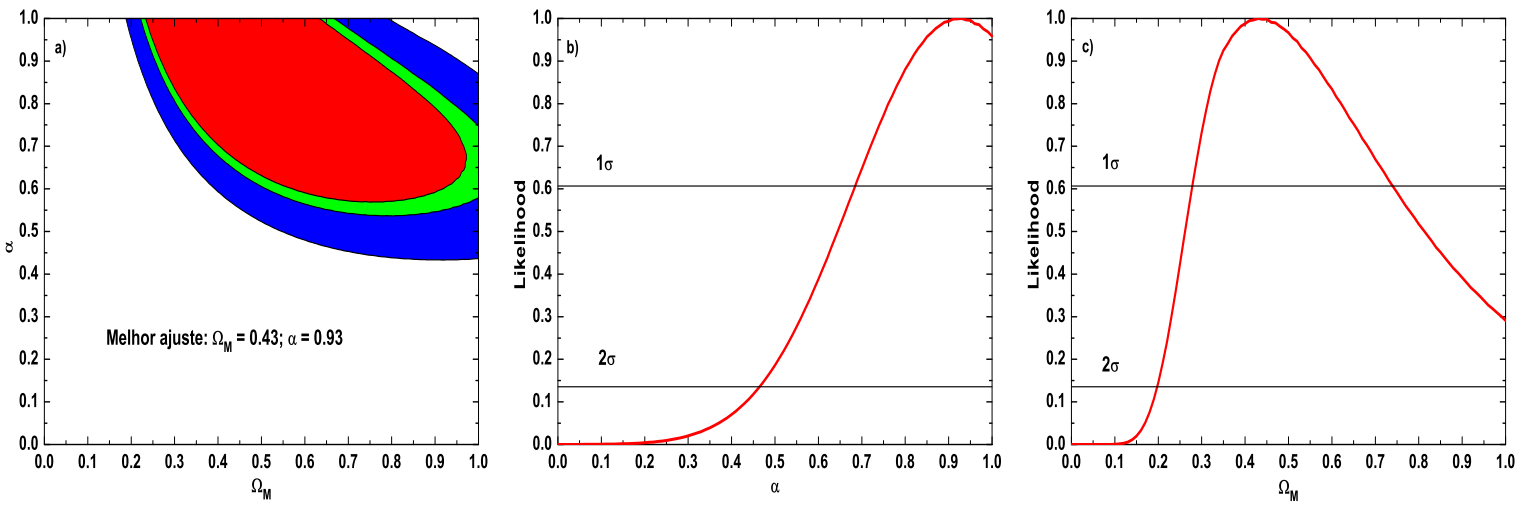

Figura 5.3: a) Plano $\Omega_{M}-\alpha$ para o modelo $\Lambda$ CDM plano obtido de 69 GRBs utilizando a calibração de Schaefer. Níveis de confiança estatística de $68.3 \%$, $90 \%$ e $95.4 \%$ estão representados na figura. b) Likelihood para o parâmetro de aglomeramento $\alpha$, onde dentro de um nível de significância de $95.4 \%$ temos que $0.47 \leq \alpha \leq 1.0$. c) Likelihood para o parâmetro de densidade da matéria $\Omega_{M}$. Para tal parâmetro obtivemos com um nível de significância de $95.4 \%$ o seguinte intervalo: $0.20 \leq \Omega_{M} \leq 1.0$.

Uma análise utilizando os 69 gamma-ray bursts calibrados para o modelo cardassiano (Mosquera Cuesta et al., 2008) foi também efetuada. Obtivemos vínculos aos parâmetros $\alpha$ e $\Omega_{M}$ através do teste $\chi^{2}$, que estão apresentados na figura 5.4. Vemos que o espaço de parâmetros pouco se modificou, mas os vínculos obtidos foram menores comparados aos obtidos utilizando a calibração de Schaefer. Os melhores ajustes são compatíveis dentro de $1 \sigma$ de confiança. Assim, concluímos que os vínculos obtidos são pouco dependentes da calibração adotada. 

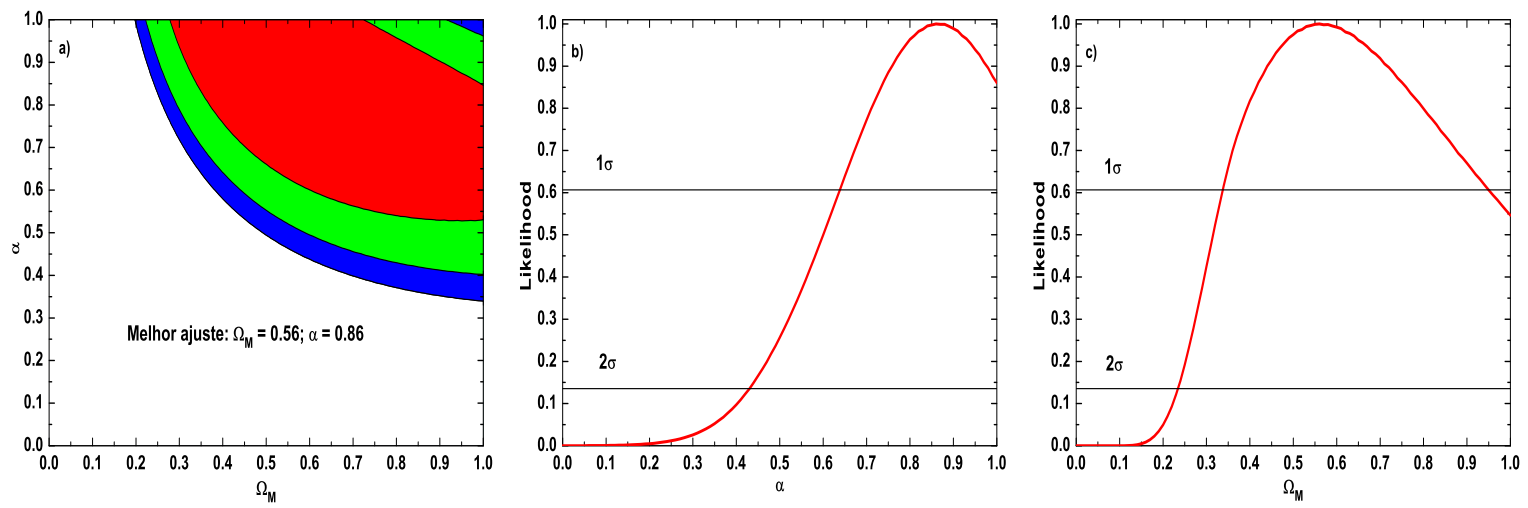

Figura 5.4: a) Contornos no plano $\Omega_{M}-\alpha$ para o modelo $\Lambda$ CDM plano obtido de GRBs utilizando a calibração para o modelo cardassiano, que representam níveis de confiança estatística de 68.3\%. 90\% e $95.4 \%$, respectivamente. b) Likelihood para o parâmetro de aglomeramento $\alpha$, onde obtivemos dentro de um nível de significância de $95.4 \%$ o intervalo $0.43 \leq \alpha \leq 1$. c) Likelihood para o parâmetro de densidade da matéria $\Omega_{M}$. Para esse parâmetro obtivemos dentro de um nível de significância de $95.4 \%$ o intervalo $0.24 \leq \Omega_{M} \leq 1$.

\subsection{Supernovas e Gamma-Ray Bursts}

É importante considerarmos uma análise conjunta para as amostras de supernovas de Kowalski et al. (2008) (Union Compilation Data) e gamma-ray bursts para a calibração de Schaefer (2007) e para o modelo cardassiano (Mosquera Cuesta et al., 2008).

Para a amostra de 307 supernovas de Kowalski et al. (2008) (Union Compilation Data) e 69 GRBs calibrados por Schaefer (2007) vinculamos os parâmetros $\alpha$ e $\Omega_{M}$ através do teste $\chi^{2}$. Os resultados obtidos são mostrados na figura 5.5. A análise conjunta é feita simplesmente somando os valores de $\chi^{2}$ para as duas amostras: $\chi^{2}=\chi_{S N}^{2}+\chi_{G R B}^{2}$, substituindo na equação (5.6) e obtendo o novo $\chi^{2}$ depois de feita a marginalização, considerando o prior dado pela equação (5.7). Percebemos que a análise conjunta permitiu a quebra de degenerescência entre os parâmetros, fazendo com que ambos fossem bem vinculados. Concluímos também que a utilização de GRBs é um instrumento valioso para vincular os parâmetros cosmológicos.

Os parâmetros $\alpha$ e $\Omega_{M}$ também foram vinculados pela utilização da amostra de 307 supernovas de Kowalski et al. (2008) (Union Compilation Data) e 69 GRBs calibrados para o modelo cardassiano (Mosquera Cuesta et al., 2008). Os vínculos obtidos estão apresentados na figura 5.6. Vemos que não houve diferença significativa ao utilizarmos a calibração para o modelo cardassiano. Logo, podemos concluir que a calibração adotada 

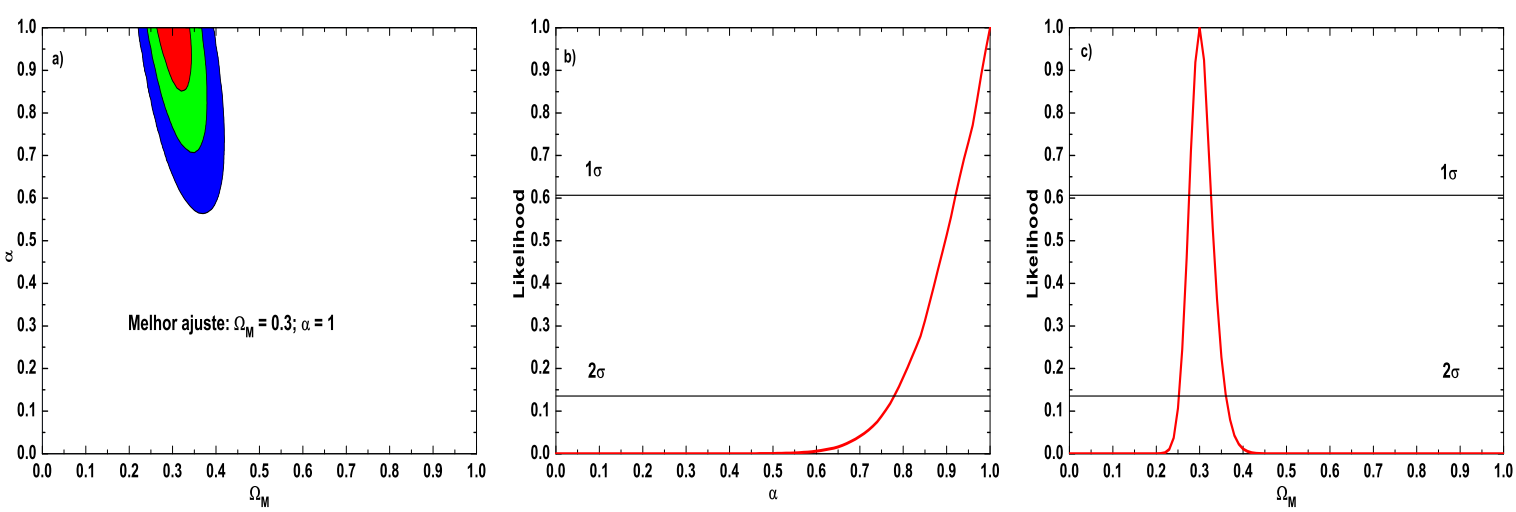

Figura 5.5: a) Plano $\Omega_{M}-\alpha$ para o modelo $\Lambda \mathrm{CDM}$ plano obtido de 307 dados de supernovas e 69 GRBs utilizando a calibração de Schaefer. Os contornos representam níveis de significância de 68.3\%, $95.4 \%$ e $99.7 \%$ respectivamente. b) Likelihood para o parâmetro de aglomeramento $\alpha$. Obtivemos para esse parâmetro dentro de um nível de significância de $95.4 \%$ o intervalo: $0.78 \leq \alpha \leq 1.0$. c) Likelihood para o parâmetro de densidade da matéria $\Omega_{M}$, onde com um nível de significância de $95.4 \%$ obtivemos $0.26 \leq \Omega_{M} \leq 0.36$.

afeta fracamente os resultados. Concluímos também que a hipótese feita para a marginalização sobre os parâmetros $a$ e $b$ obtidos da calibração mostrou-se consistente, uma vez que não foi observada uma diferença considerável entre as calibrações. Por fim, sendo o resultado mais importante desta dissertação, vemos que a análise conjunta quebrou a degenerescência entre os parâmetros, permitindo que ambos fossem bem vinculados. Esses resultados serão brevemente submetidos a publicação (Busti et al., 2009a).

Na tabela 5.1 sintetizamos os resultados obtidos quando consideramos um prior gaussiano sobre $H_{0}$. Para a amostra de supernovas, vimos que $\Omega_{M}$ foi bem vinculado, consistente com testes independentes, diferentemente do vínculo para $\alpha$, que foi fracamente vinculado. Para os dados de gamma-ray bursts, o parâmetro $\Omega_{M}$ foi fracamente vinculado, o que pode ser entendido pela percepção de que dados em altos redshifts não vinculam a energia escura. As duas calibrações consideradas propiciaram vínculos semelhantes, o que nos permite dizer que tais são fracamente dependentes da calibração adotada. Já a análise conjunta possibilitou a quebra de degenerescência entre os parâmetros, que foram bem limitados dentro de $95.4 \%$ de confiança estatística. 

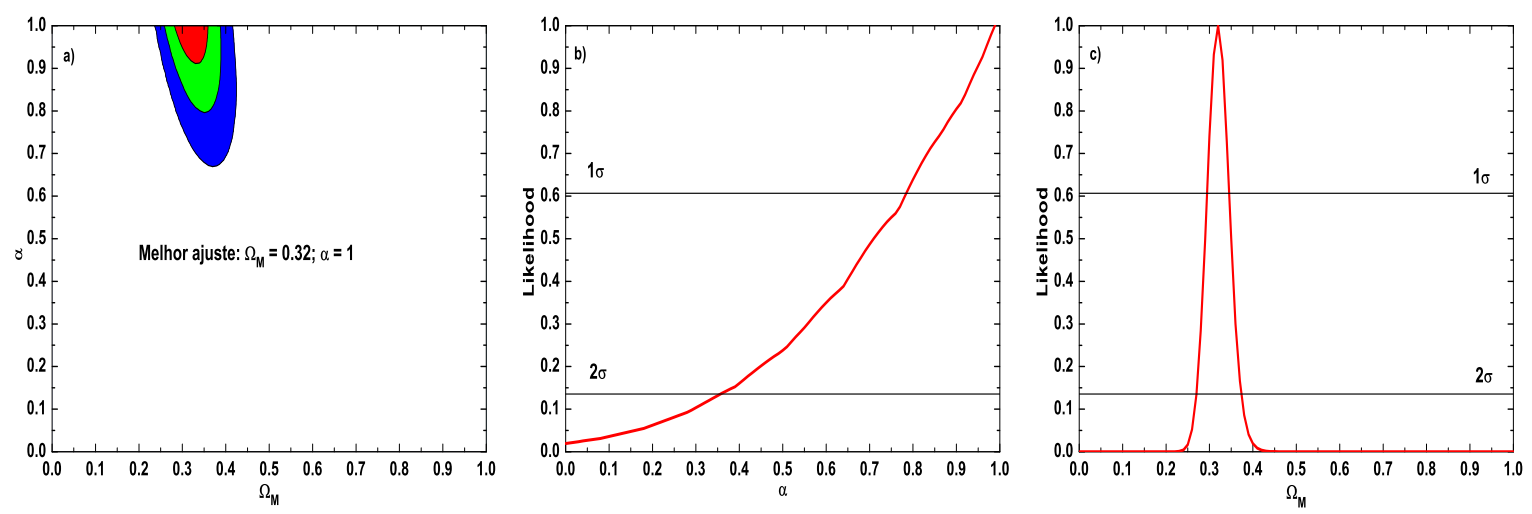

Figura 5.6: a) Plano $\Omega_{M}-\alpha$ para o modelo $\Lambda$ CDM plano obtido de 307 dados de supernovas e 69 GRBs utilizando a calibração para o modelo cardassiano. Os contornos representam níveis de significância de $68.3 \%, 95.4 \%$ e $99.7 \%$ respectivamente. b) Likelihood para o parâmetro de aglomeramento $\alpha$. Dentro de um nível de significância de $95.4 \%$ obtivemos o intervalo $0.86 \leq \alpha \leq 1$. c) Likelihood para o parâmetro de densidade da matéria $\Omega_{M}$, onde obtivemos dentro de um nível de significância de $95.4 \%$ o intervalo $0.28 \leq \Omega_{M} \leq 0.37$.

Tabela 5.1 - Vínculos para os parâmetros $\alpha$ e $\Omega_{M}$ considerando um prior gaussiano em $H_{0}$. $\chi_{\min }^{2}$ representa o $\chi^{2}$ mínimo obtido, o subscrito $M A$ representa o melhor ajuste obtido e $2 \sigma$ o intervalo de confiança estatística de 95.4\%. (S) representa os GRBs calibrados por Schaefer (2007) e (C) os GRBs calibrados para o modelo cardassiano (Mosquera Cuesta et al., 2008).

\begin{tabular}{lccccc}
\hline \hline \multicolumn{1}{c}{ Amostra } & $\chi_{\text {min }}^{2}$ & $\alpha_{M A}$ & $\left(\Omega_{M}\right)_{M A}$ & $2 \sigma$ & $2 \sigma$ \\
\hline SN & 312.38 & 1 & 0.29 & $0.36 \leq \alpha \leq 1.0$ & $0.24 \leq \Omega_{M} \leq 0.37$ \\
GRB(S) & 70.65 & 0.93 & 0.43 & $0.47 \leq \alpha \leq 1.0$ & $0.20 \leq \Omega_{M} \leq 1.0$ \\
GRB(C) & 70.48 & 0.86 & 0.56 & $0.43 \leq \alpha \leq 1.0$ & $0.24 \leq \Omega_{M} \leq 1.0$ \\
SN e GRB(S) & 383.73 & 1 & 0.30 & $0.78 \leq \alpha \leq 1.0$ & $0.26 \leq \Omega_{M} \leq 0.36$ \\
SN e GRB(C) & 388.00 & 1 & 0.32 & $0.86 \leq \alpha \leq 1.0$ & $0.28 \leq \Omega_{M} \leq 0.37$ \\
& & & & & \\
\hline
\end{tabular}


Capítulo 6

\section{Vínculos Observacionais para $\alpha$ e $\gamma$}

\subsection{Introdução}

Neste capítulo analisaremos os vínculos obtidos ao modelo com criação de matéria. Pelas conclusões obtidas no capítulo anterior, consideraremos apenas a calibração de Schaefer, uma vez que tanto essa calibração quanto a calibração para o modelo cardassiano propiciaram vínculos semelhantes nas quantidades de interesse cosmológico. Restringimos nossa análise ao prior gaussiano sobre $H_{0}$, já que esse prior reflete de forma mais confiável os vínculos observacionais. Pelos mesmos argumentos expostos, a hipótese sobre a marginalização dos $a_{j}$ e $b_{j}$ é mantida, ou seja, consideraremos o melhor ajuste das relações fenomenológicas.

No tratamento a seguir apenas o caso $\beta=0$ será considerado, por se tratar do modelo mais simples apresentando transição entre desaceleração e aceleração em baixos redshifts. Além disso, $\beta=0$ foi o melhor ajuste obtido pela análise de supernovas realizada por Lima et al. (2008). O parâmetro de Hubble neste caso se reduz para (ver equação (3.25))

$$
H(z)=H_{0}\left[\gamma+(1-\gamma)(1+z)^{\frac{3}{2}}\right]
$$

Assumindo $\beta=0$, a equação ZKDR para a distância de diâmetro angular é bastante simplificada, sendo expressa por:

$$
F \frac{d^{2} D_{A}}{d z^{2}}+G \frac{d D_{A}}{d z}+H D_{A}=0
$$

onde 


$$
\begin{gathered}
F=\gamma(1+z)^{2}+(1-\gamma)(1+z)^{\frac{7}{2}}, \\
G=\frac{7}{2}(1-\gamma)(1+z)^{\frac{5}{2}}+2 \gamma(1+z), \\
H=\frac{3 \alpha}{2}(1-\gamma)(1+z)^{\frac{3}{2}} .
\end{gathered}
$$

Considerando $\beta=0$ na equação (4.46), a equação ZKDR para a distância de luminosidade toma a seguinte forma:

$$
\mathcal{F} \frac{d^{2} D_{L}}{d z^{2}}-\mathcal{G} \frac{d D_{L}}{d z}+\mathcal{H} D_{L}=0
$$

onde $\mathcal{F}, \mathcal{G}$ e $\mathcal{H}$ são funções dos parâmetros cosmológicos, expressos em termos do redshift por:

$$
\begin{aligned}
\mathcal{F} & =\gamma+(1-\gamma)(1+z)^{\frac{3}{2}} \\
\mathcal{G} & =2 \gamma(1+z)^{-1}+\frac{1}{2}(1-\gamma)(1+z)^{\frac{1}{2}} \\
\mathcal{H} & =\left(\frac{3 \alpha-2}{2}\right)(1-\gamma)(1+z)^{-\frac{1}{2}}+2 \gamma(1+z)^{-2} .
\end{aligned}
$$

O módulo de distância em função da distância de luminosidade é dado por (Peebles, 1993):

$$
\mu=5 \log \left(D_{L}\right)+25
$$

onde $D_{L}$ é expressa em Mpc.

Com as hipóteses adotadas, o $\chi^{2}$ para o modelo com criação de matéria pode ser escrito como:

$$
\chi^{2}=\sum_{i}^{N} \frac{\left(\mu_{\text {teor }}\left(z_{i}, \alpha, \gamma, H_{0}\right)-\mu_{i}\left(z_{i}\right)\right)^{2}}{\sigma_{\mu_{i}}^{2}},
$$


onde $\mu_{\text {teor }}$ é o módulo de distância teórico calculado a partir de (6.4) e (6.6), $\mu_{i}$ é o módulo de distância obtido a partir dos dados observacionais, $\sigma_{\mu_{i}}$ é a incerteza do módulo de distância e $N$ é o número de dados.

Como consideramos um prior gaussiano, a nova Likelihood é

$$
\tilde{\mathcal{L}}=\int e^{-\frac{\chi^{2}}{2}} \pi\left(H_{0}\right) d H_{0}
$$

onde o prior $\pi\left(H_{0}\right)$ é representado por

$$
\pi\left(H_{0}\right)=e^{-\frac{1}{2}\left(\frac{H_{0}-72}{8}\right)^{2}}
$$

sendo $H_{0}$ dado em $\mathrm{Km} \mathrm{s}^{-1} \mathrm{Mpc}^{-1}$.

Temos agora o novo $\chi^{2}$ expresso na seguinte forma

$$
\tilde{\chi}^{2}=-2 \ln \tilde{\mathcal{L}}
$$

Na figura 6.1 são apresentadas as magnitudes residuais comparadas a um universo vazio para vários valores dos parâmetros $\gamma$ e $\alpha$, assim como para o modelo $\Lambda$ CDM, com os dados de supernova tipo Ia de Kowalski et al. (2008) e dados de GRBs de Schaefer (2007).
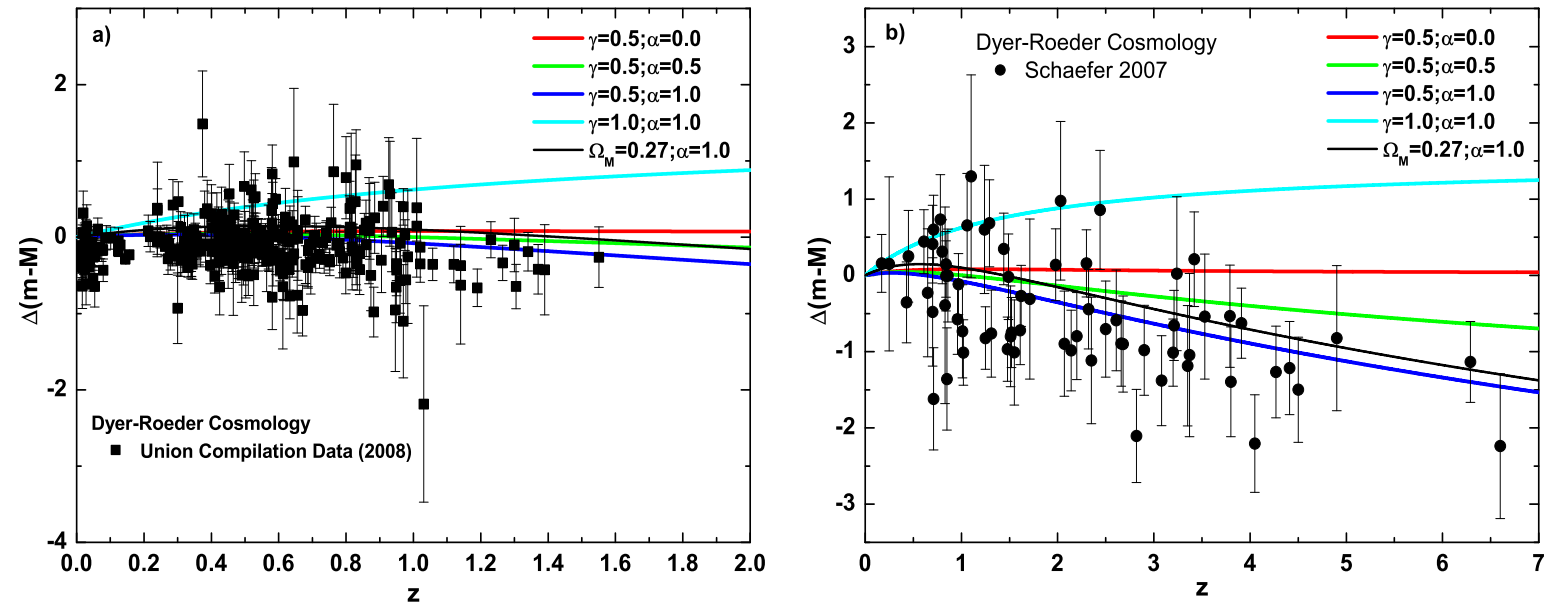

Figura 6.1: Magnitude residual comparada a um universo vazio $\left(\Omega_{M}=0, \Omega_{\Lambda}=0\right)$ para: a) 307 supernovas da amostra de Kowalski et al. (2008) (Union Compilation Data) b) 69 GRBs calibrados por Schaefer (2007). 


\subsection{Supernovas}

Utilizando as 307 supernovas da amostra de Kowalski et al. (2008) (Union Compilation Data) vinculamos os parâmetros $\alpha$ e $\gamma$ pelo teste $\chi^{2}$. Os resultados obtidos estão apresentados na figura 6.2. Vemos que o parâmetro $\gamma$ foi bem vinculado dentro de $2 \sigma$ de confiança: $0.57 \leq \gamma \leq 0.70$. O mesmo não ocorreu para o parâmetro $\alpha$, que foi vinculado em $\alpha \geq 0.4$ com $2 \sigma$ de confiança estatística. O melhor ajuste obtido $(\gamma=0.64$ e $\alpha=1)$ é compatível com um universo homogêneo e isotrópico em todas as escalas, sendo a magnitude de $\gamma$ próxima ao valor $\gamma=0.7$ obtido por Lima et al. (2008).
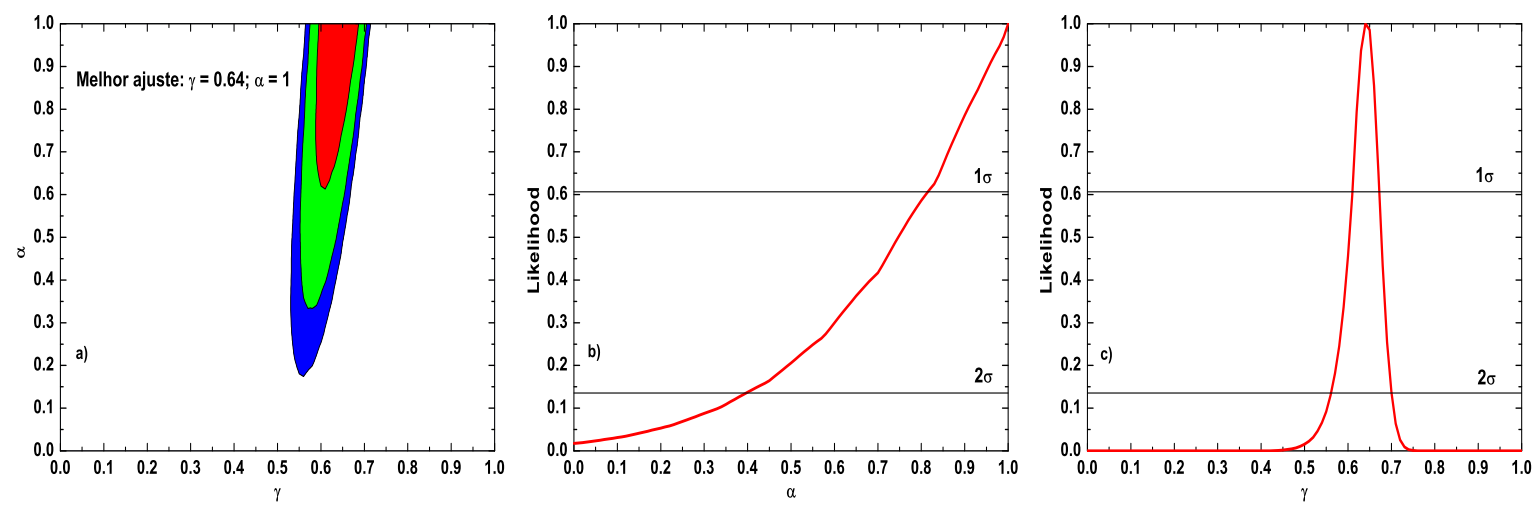

Figura 6.2: a) Plano $\gamma-\alpha$ para o modelo com criação de matéria obtido de 307 dados de supernovas. Os contornos representam níveis de confiança estatística de $68.3 \%, 90 \%$ e $95.4 \%$ respectivamente. b) Likelihood para o parâmetro de aglomeramento $\alpha$, onde dentro de um nível de significância de $95.4 \%$ obtivemos o intervalo $0.40 \leq \alpha \leq 1.0$ c) Likelihood para o parâmetro $\gamma$. Dentro de um nível de significância de $95.4 \%$ obtivemos $0.57 \leq \gamma \leq 0.70$.

\subsection{Gamma-Ray Bursts}

A amostra de 69 GRBs calibrados por Schaefer (2007) foi utilizada para vincular os parâmetros $\alpha$ e $\gamma$ através do teste $\chi^{2}$. Os resultados obtidos estão apresentados na figura 6.3. Para o parâmetro $\gamma$ uma grande região do espaço de parâmetros é permitida dentro de $2 \sigma$ de confiança. Já para o parâmetro $\alpha$ o vínculo obtido é semelhante ao obtido com supernovas. O melhor ajuste para o parâmetro $\alpha$ foi o mesmo, já para o parâmetro $\gamma$ foi mais baixo, igual a 0.46 . Com $2 \sigma$ de confiança estatística os valores de $\gamma$ e $\alpha$ estão restritos aos intervalos $0 \leq \gamma \leq 0.65$ e $0.46 \leq \alpha \leq 1.0$, respectivamente. Comparando os vínculos obtidos pelos dois conjuntos de dados, vemos que os de gamma-ray bursts são 
menos restritivos do que os de supernovas.
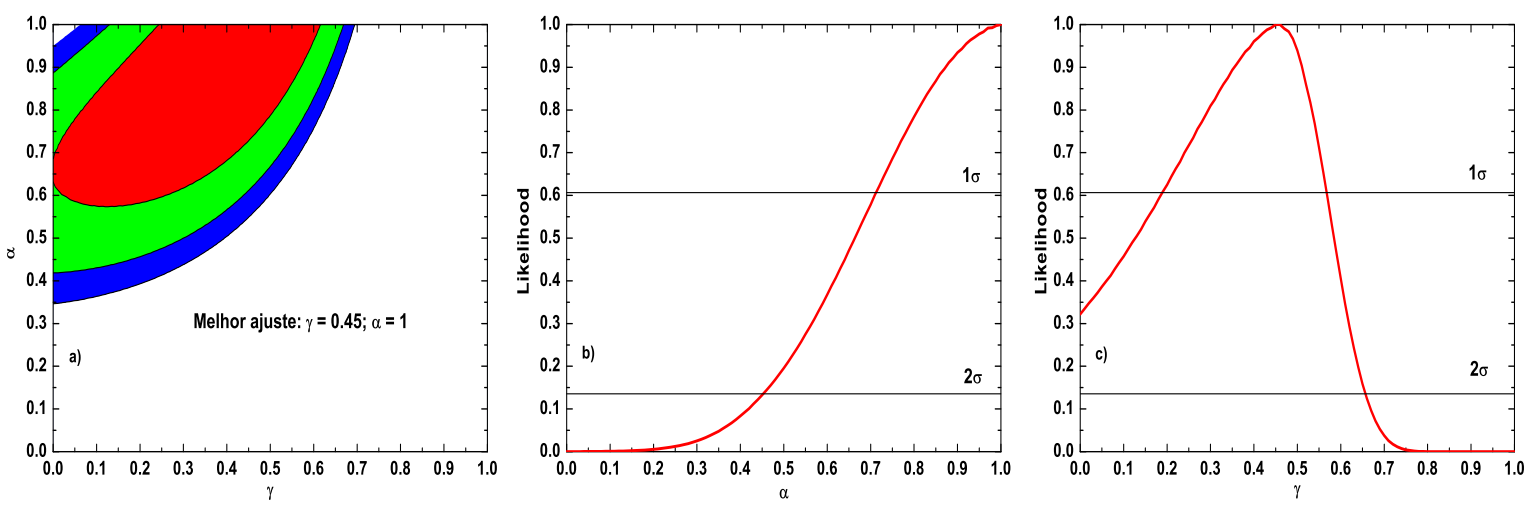

Figura 6.3: a) Contornos no plano $\gamma-\alpha$ para o modelo com criação de matéria obtido de 69 GRBs utilizando a calibração de Schaefer, onde representam níveis de confiança estatística de $68.3 \%$, 90\% e $95.4 \%$ respectivamente. b) Likelihood para o parâmetro de aglomeramento $\alpha$. Com um nível de significância de 95.4\% obtivemos o intervalo $0.46 \leq \alpha \leq 1.0$. c) Likelihood para o parâmetro $\gamma$. Obtivemos dentro de um nível de significância de $95.4 \%$ o intervalo $0 \leq \gamma \leq 0.65$.

\subsection{Supernovas e Gamma-Ray Bursts}

Vamos agora considerar para o modelo de criação de matéria uma análise conjunta envolvendo os dados de supernovas e gamma-ray bursts. Novamente analisamos as 307 supernovas da amostra de Kowalski et al. (2008) (Union Compilation Data) e os 69 GRBs calibrados por Schaefer (2007).

Os resultados obtidos estão apresentados na figura 6.4. Para a análise conjunta é suficiente somar os valores de $\chi^{2}: \chi^{2}=\chi_{S N}^{2}+\chi_{G R B}^{2}$. Tal análise permitiu obter vínculos mais restritivos para os parâmetros de interesse físico. Com $2 \sigma$ de confiança estatística, ambos parâmetros foram vinculados sobre os intervalos: $0.85 \leq \alpha \leq 1$ e $0.56 \leq \gamma \leq 0.66$. Vemos que a combinação das duas amostras independentes é uma ferramenta importante na determinação dos parâmetros cosmológicos.

Na tabela 6.1 compilamos os resultados obtidos para o modelo com criação de matéria, tanto para as análises individuais de supernovas e gamma-ray bursts quanto para a análise conjunta. Esses resultados serão brevemente submetidos a publicação (Busti et al., 2009b). O espaço de parâmetros obtido pela análise só de supernovas ou gamma-ray bursts foi parecido, a diferença mais significativa concerne ao bom vínculo ao parâmetro $\gamma$ obtido 
com supernovas. Já a análise conjunta mostra os resultados mais satisfatórios, visto que ela quebra a degenerescência entre os parâmetros fazendo com que ambos sejam bem limitados.
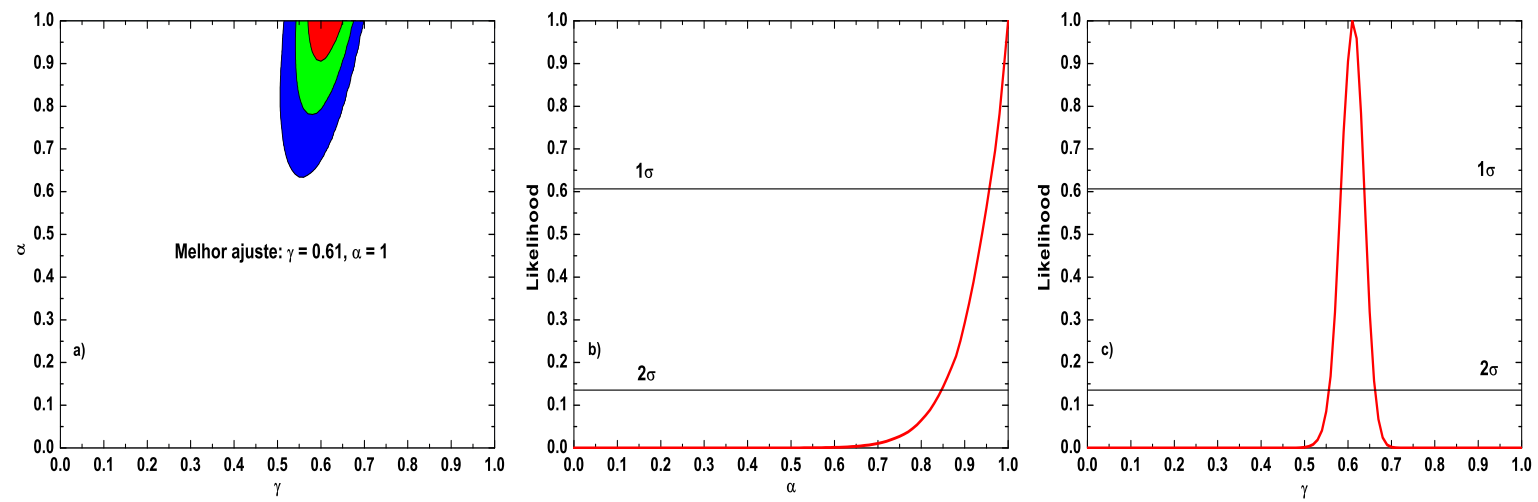

Figura 6.4: a) Plano $\gamma-\alpha$ para o modelo com criação de matéria obtido de 307 dados de supernovas e 69 GRBs utilizando a calibração de Schaefer. Os contornos representam níveis de significância de 68.3\%, 95.4\% e 99.7\% respectivamente. b) Likelihood para o parâmetro de aglomeramento $\alpha$. Dentro de um nível de significância de $95.4 \%$ obtivemos o intervalo $0.85 \leq \alpha \leq 1.0$. c) Likelihood para o parâmetro $\gamma$, onde obtivemos dentro de um nível de significância de $95.4 \%$ o intervalo $0.56 \leq \gamma \leq 0.66$.

Tabela 6.1 - Vínculos para os parâmetros $\alpha$ e $\gamma$ considerando um prior gaussiano em $H_{0}$. $\chi_{\min }^{2}$ representa o $\chi^{2}$ mínimo obtido, o subscrito $M A$ representa o melhor ajuste obtido e $2 \sigma$ o intervalo de confiança estatística de $95.4 \%$.

\begin{tabular}{lccccc}
\hline \hline Amostra & $\chi_{\text {min }}^{2}$ & $\alpha_{M A}$ & $\gamma_{M A}$ & $2 \sigma$ & $2 \sigma$ \\
\hline \multirow{2}{*}{ SN } & 314.75 & 1 & 0.64 & $0.40 \leq \alpha \leq 1.0$ & $0.57 \leq \gamma \leq 0.70$ \\
GRB & 70.77 & 1 & 0.45 & $0.46 \leq \alpha \leq 1.0$ & $0 \leq \gamma \leq 0.65$ \\
SN e GRB & 389.71 & 1 & 0.61 & $0.85 \leq \alpha \leq 1.0$ & $0.56 \leq \gamma \leq 0.66$
\end{tabular}




\section{Capítulo 7}

\section{Conclusões e Perspectivas}

Na última década, o acúmulo de dados observacionais indica que o Universo se encontra numa fase de expansão acelerada cujo mecanismo subjacente permanece ainda desconhecido, sendo sua identificação atualmente considerada o problema central da cosmologia moderna. No contexto da relatividade geral, a maneira mais simples de acelerar o universo é supor a existência de uma componente extra (em adição a matéria escura) cuja característica principal é ter pressão negativa. No entanto, mesmo no âmbito da relatividade geral, existem outras possibilidades, como por exemplo, o processo de criação de matéria escura pelo campo gravitacional variável do universo, cujas conseqüências físicas apenas recentemente começaram a ser debatidas na literatura.

A maneira mais prática de estudarmos a influência dos constituintes cosmológicos é através dos efeitos gravitacionais que ficam impressos na história da evolução do universo (os chamados testes observacionais). Vários deles estão diretamente relacionados com a noção de distâncias, cuja determinação é sempre um problema crucial em cosmologia. É exatamente este ponto que discutimos em nosso projeto de mestrado.

Como foi discutido, a luz em sua trajetória sente as inomogeneidades locais. Para um universo homogêneo apenas em grande escala, as inomogeneidades locais alteram as distâncias calculadas quando consideramos um modelo do tipo FLRW. Como vimos, os desvios em relação ao caso homogêneo podem ser fenomenologicamente tratados com base no parâmetro de aglomeramento $\alpha$, que quantifica a magnificação sofrida pelo feixe de luz ao longo do seu percurso. Numa teoria métrica da gravitação, as distâncias modificadas podem ser calculadas através da chamada equação diferencial ZKDR (ZeldovichKantowski-Dyer-Roeder). Naturalmente, como as inomogeneidades alteram as distâncias, 
os parâmetros cosmológicos também são afetados. É neste contexto que se inseriu a presente dissertação. Estudamos o efeito do parâmetro de aglomeramento $\alpha$ para o modelo $\Lambda \mathrm{CDM}$ plano e para o modelo com criação de matéria escura, recentemente proposto por Lima e colaboradores (2008).

Como foi visto, no primeiro capítulo descrevemos brevemente o modelo padrão da cosmologia. Iniciamos por sua base teórica, a teoria da relatividade geral. Depois, vimos que a imposição de um universo homogêneo e isotrópico leva à métrica do tipo FLRW e finalmente discutimos a dinâmica cósmica.

No segundo capítulo rediscutimos a escada de distância cósmica, estudando em detalhe os indicadores primários e secundários de distância. Discutimos as definições de distância quando levamos em consideração o efeito da expansão do universo. Finalmente, descrevemos o método baseado na utilização de gamma-ray bursts como velas padrão.

Sabemos que a natureza da energia escura constitui o maior desafio da cosmologia moderna. Assim, no terceiro capítulo, descrevemos os modelos que cujas previsões foram mais analisadas na literatura. Uma atenção especial foi dedicada aos modelos relativísticos para os quais a inclusão da energia escura não é necessária, tal como ocorre no no modelo cardassiano e em modelos com criação de matéria escura fria.

Os efeitos das inomogeneidades foram estudados no quarto capítulo. Primeiramente, deduzimos a equação óptica de Sachs, que é uma equação diferencial governando a evolução da área transversal de um feixe de luz ao longo de sua trajetória no espaço-tempo. A partir desta equação deduzimos a equação ZKDR, uma equação diferencial que relaciona a distância, seja a de diâmetro angular ou luminosidade, com o efeito de aglomeramento dado pelo parâmetro $\alpha$. Obtivemos a equação ZKDR para o modelo $\Lambda$ CDM plano e para o modelo com criação de matéria.

No quinto capítulo apresentamos os vínculos obtidos aos parâmetros $\alpha$ e $\Omega_{M}$ para o modelo $\Lambda$ CDM plano. Utilizamos a amostra de 307 supernovas de Kowalski et al. (2008) (Union Compilation Data) e 69 gamma-ray bursts calibrados por Schaefer (2007) e calibrados para o modelo cardassiano (Mosquera Cuesta et al., 2008). As análises foram feitas considerando um prior gaussiano sobre $H_{0}$. O teste $\chi^{2}$ foi efetuado para cada amostra separadamente, assim como para uma análise conjunta de supernovas e GRBs.

Verificamos que os vínculos obtidos são fracamente dependentes da calibração ado- 
tada. Vimos também que a análise conjunta permitiu quebrar a degenerescência entre os parâmetros, fazendo com que ambos os parâmetros fossem bem vinculados. Com um prior gaussiano obtivemos os seguintes intervalos de confiança para a análise conjunta com supernovas e os GRBs calibrados por Schaefer (2007): $0.78 \leq \alpha \leq 1.0(2 \sigma)$ e $0.26 \leq \Omega_{M} \leq 0.36(2 \sigma)$. Os resultados evidenciam a complementaridade do método baseado em gamma-ray bursts a outros testes observacionais, uma vez que tal método nos permite acessar objetos que estão em redshifts $z \sim 6$.

No sexto capítulo apresentamos os vínculos obtidos sobre os parâmetros $\alpha$ e $\gamma$, este último caracterizando um modelo com criação de matéria escura fria. Aqui também utilizamos a amostra de 307 supernovas do Union Compilation Data Kowalski et al. (2008) e 69 gamma-ray bursts calibrados por Schaefer (2007). Consideramos novamente um prior gaussiano, sendo o teste $\chi^{2}$ primeiro realizado para cada amostra separadamente e depois numa análise conjunta.

Da mesma forma do capítulo 5, verificamos que a análise conjunta permitiu quebrar a degenerescência entre os parâmetros, fazendo que ambos fossem bem vinculados. Os limites com $95.4 \%$ de significância estatística para a análise conjunta foram: $0.85 \leq \alpha \leq 1.0$ e $0.56 \leq \gamma \leq 0.66$

Resumindo, a contribuição científica mais interessante desta dissertação se relaciona com os vínculos obtidos para alguns parâmetros cosmológicos no contexto de modelos localmente inomogêneos. Os resultados originais estão apresentados nos capítulos 5 e 6 e no apêndice B. Os limites foram obtidos através de uma análise conjunta envolvendo dados de Supernovas Ia e Gamma-Ray Bursts, com as inomogeneidades locais ocorrendo devido ao processo de formação de estruturas e quantificadas (na fase não linear) pelo parâmetro de aglomeramento $\alpha$.

Como perspectiva futura para o presente estudo, é importante investigar a dependência do parâmetro de aglomeramento $\alpha$ com a direção e também com o redshift, assim como a influência do tensor de cisalhamento $\left(\sigma_{a b}\right)$ na determinação das distâncias, cuja implementação deverá alterar os limites obtidos no presente trabalho ( $\alpha$ constante e tensor de cisalhamento nulo). É interessante também considerar que a amostra de Gamma-Ray Bursts pode ser calibrada por Supernovas em redshifts baixos e intermediários (mesma distância), o que certamente vinculará os parâmetros cosmológicos relevantes com mais 
precisão. Naturalmente, com a enorme quantidade de dados previstos para os projetos observacionais dos próximos anos (como por exemplo PLANCK, SNAP, LSST), será possível utilizar testes estatísticos mais robustos para uma possível análise conjunta envolvendo todas as amostras independentes de dados cosmológicos.

Finalmente, acreditamos que a utilização da distância ZKDR pode se revelar uma ferramenta importante para vincular mais precisamente os os parâmetros livres dos modelos (com e sem energia escura), em harmonia com a era da precisão cosmológica que atualmente vivenciamos. 


\section{Referências Bibliográficas}

Abramo L. R. W., Lima J. A. S., Inflationary models driven by adiabatic matter creation., Classical and Quantum Gravity, 1996, vol. 13, p. 2953

Alcaniz J. S., Lima J. A., Interpreting cosmological vacuum decay, Phys. Rev. D, 2005a, vol. 72 , p. 063516

Alcaniz J. S., Lima J. A. S., Closed and open FRW cosmologies with matter creation: kinematic tests, A\&A, 1999a, vol. 349, p. 729

Alcaniz J. S., Lima J. A. S., New Limits on $\Omega_{\Lambda}$ and $\Omega_{M}$ from Old Galaxies at High Redshift, ApJ, 1999b, vol. 521, p. L87

Alcaniz J. S., Lima J. A. S., Dark Energy and the Epoch of Galaxy Formation, ApJ, 2001, vol. 550, p. L133

Alcaniz J. S., Lima J. A. S., Measuring the Chaplygin Gas Equation of State from Angular and Luminosity Distances, ApJ, 2005b, vol. 618, p. 16

Alcaniz J. S., Lima J. A. S., Cunha J. V., Cosmological implications of APM 08279+5255, an old quasar at $\mathrm{z}=3.91$, MNRAS, 2003, vol. 340, p. L39

Alcaniz J. S., Lima J. A. S., Silva R., Mass Inhomogeneities and the Angular Size-Redshift Relation, International Journal of Modern Physics D, 2004, vol. 13, p. 1309

Allen S. W., Rapetti D. A., Schmidt R. W., Ebeling H., Morris R. G., Fabian A. C., Improved constraints on dark energy from Chandra X-ray observations of the largest relaxed galaxy clusters, MNRAS, 2008, vol. 383, p. 879 
Allen S. W., Schmidt R. W., Fabian A. C., Cosmological constraints from the X-ray gas mass fraction in relaxed lensing clusters observed with Chandra, MNRAS, 2002, vol. 334, p. L11

Alpher R. A., Bethe H., Gamow G., The Origin of Chemical Elements, Physical Review, 1948, vol. 73 , p. 803

Alpher R. A., Herman R., Gamow G. A., Thermonuclear Reactions in the Expanding Universe, Physical Review, 1948, vol. 74, p. 1198

Amendola L., Finelli F., Burigana C., Carturan D., WMAP and the generalized Chaplygin gas, Journal of Cosmology and Astro-Particle Physics, 2003, vol. 7, p. 5

Arkani-Hamed N., Dimopoulos S., Dvali G., Phenomenology, astrophysics, and cosmology of theories with submillimeter dimensions and TeV scale quantum gravity, Phys. Rev. D, 1999, vol. 59, p. 086004

Bento M. C., Bertolami O., Sen A. A., Generalized Chaplygin gas, accelerated expansion, and dark-energy-matter unification, Phys. Rev. D, 2002, vol. 66, p. 043507

Bildhauer S., Futamase T., The cosmic microwave background in a globally inhomogeneous universe, MNRAS, 1991, vol. 249, p. 126

Bilić N., Tupper G. B., Viollier R. D., Unification of dark matter and dark energy: the inhomogeneous Chaplygin gas, Physics Letters B, 2002, vol. 535, p. 17

Bronstein M. P., On the expanding universe, Phys. Z. Sowjetunion, 1933, vol. 3, p. 73

Buchalter A., Helfand D. J., Becker R. H., White R. L., Constraining Omega 0 with the Angular Size-Redshift Relation of Double-lobed Quasars in the FIRST Survey, ApJ, 1998, vol. 494, p. 503

Buchert T., Ehlers J., Averaging inhomogeneous Newtonian cosmologies., A\&A, 1997, vol. 320 , p. 1

Busti V. C., Santos R. C., Lima J. A. S., Constraining the Smoothness Parameter $\alpha$ with Supernovae Ia and Gamma-Ray Bursts, em preparação, 2009a 
Busti V. C., Santos R. C., Lima J. A. S., Constraining the Smoothness Parameter $\alpha$ with Supernovae Ia and Gamma-Ray Bursts for an Accelerating CDM Cosmology, em preparação, 2009b

Caldwell R. R., A phantom menace? Cosmological consequences of a dark energy component with super-negative equation of state, Physics Letters B, 2002, vol. 545, p. 23

Calvão M. O., Waga I., Lima J. A. S., On the thermodynamics of matter creation in cosmology., Physics Letters A, 1992, vol. 162, p. 223

Capozziello S., Carloni S., Troisi A., Quintessence without scalar fields, astro-ph/0303041, 2003

Carneiro S., Lima J. A. S., Time Dependent Cosmological Term and Holography, International Journal of Modern Physics A, 2005, vol. 20, p. 2465

Carroll S. M., Duvvuri V., Trodden M., Turner M. S., Is cosmic speed-up due to new gravitational physics?, Phys. Rev. D, 2004, vol. 70, p. 043528

Carvalho F. C., Alcaniz J. S., Lima J. A. S., Silva R., Scalar-Field-Dominated Cosmology with a Transient Acceleration Phase, Physical Review Letters, 2006, vol. 97, p. 081301

Carvalho F. C., Alcaniz J. S., Lima J. A. S., Silva R., CMB and LSS constraints on a single-field model of inflation, Europhysics Letters, 2008, vol. 83, p. 29001

Cavallo G., Rees M. J., A qualitative study of cosmic fireballs and gamma-ray bursts, MNRAS, 1978, vol. 183, p. 359

Chevallier M., Polarski D., Accelerating Universes with Scaling Dark Matter, International Journal of Modern Physics D, 2001, vol. 10, p. 213

Chung D. J. H., Freese K., Cosmological challenges in theories with extra dimensions and remarks on the horizon problem, Phys. Rev. D, 2000, vol. 61, p. 023511

Cole S., Percival W. J., Peacock J. A., Norberg P., et al. The 2dF Galaxy Redshift Survey: power-spectrum analysis of the final data set and cosmological implications, MNRAS, 2005, vol. 362, p. 505 
Combes F., Boisse P., Mazure A., Blanchard A., Seymour M., Galaxies and cosmology. Galaxies and cosmology (2nd ed.). by F. Combes et al. (M. Seymour, Trans.). New York: Springer, 2002., 2002

Copeland E. J., Sami M., Tsujikawa S., Dynamics of Dark Energy, International Journal of Modern Physics D, 2006, vol. 15, p. 1753

Costa E., Frontera F., Heise J., et al. Discovery of an X-ray afterglow associated with the $\gamma$-ray burst of 28 February 1997, Nature, 1997, vol. 387, p. 783

Cunha J. V., Energia Escura: Origem, Candidatos e VÃnculos Observacionais, Universidade Federal do Rio Grande do Norte, 2006, Tese de Doutorado, 172 p.

Cunha J. V., Alcaniz J. S., Lima J. A., Cosmological constraints on Chaplygin gas dark energy from galaxy cluster x-ray and supernova data, Phys. Rev. D, 2004, vol. 69, p. 083501

Cunha J. V., Lima J. A. S., Pires N., Deflationary Lambda (t) cosmology: Observational expressions, A\&A, 2002, vol. 390, p. 809

Cunha J. V., Marassi L., Lima J. A. S., Constraining $H_{0}$ from the Sunyaev-Zel'dovich effect, galaxy cluster X-ray data and baryon oscillations, MNRAS, 2007, vol. 379, p. L1

Cunha J. V., Santos R. C., The Existence of AN Old Quasar at $\mathrm{z}=3.91$ and its Implications for $\Lambda(\mathrm{t})$ Deflationary Cosmologies, International Journal of Modern Physics D, 2004, vol. 13, p. 1321

Daly R. A., Guerra E. J., Quintessence, Cosmology, and Fanaroff-Riley Type IIb Radio Galaxies, AJ, 2002, vol. 124, p. 1831

Damour T., Ruffini R., Quantum electrodynamical effects in Kerr-Newman geometries., Physical Review Letters, 1975, vol. 35, p. 463

de Sitter W., Einstein's theory of gravitation and its astronomical consequences. Third paper, MNRAS, 1917, vol. 78, p. 3 
Demianski M., de Ritis R., Marino A. A., Piedipalumbo E., Approximate angular diameter distance in a locally inhomogeneous universe with nonzero cosmological constant, A\&A, 2003, vol. 411, p. 33

Djorgovski S., Davis M., Fundamental properties of elliptical galaxies, ApJ, 1987, vol. 313, p. 59

Dressler A., Lynden-Bell D., Burstein D., Davies R. L., Faber S. M., Terlevich R., Wegner G., Spectroscopy and photometry of elliptical galaxies. I - A new distance estimator, ApJ, 1987, vol. 313, p. 42

Dyer C. C., Roeder R. C., The Distance-Redshift Relation for Universes with no Intergalactic Medium, ApJ, 1972, vol. 174, p. L115

Dyer C. C., Roeder R. C., Distance-Redshift Relations for Universes with Some Intergalactic Medium, ApJ, 1973, vol. 180, p. L31

Dyer C. C., Roeder R. C., Observations in Locally Inhomogeneous Cosmological Models, ApJ, 1974, vol. 189, p. 167

Eichler D., Levinson A., An Interpretation of the $h \nu_{\text {peak }}-E_{\text {iso }}$ Correlation for Gamma-Ray Bursts, ApJ, 2004, vol. 614, p. L13

Einstein A., Kosmologische Betrachtungen zur allgemeinen Relativitätstheorie, Sitzungsberichte der Königlich Preußischen Akademie der Wissenschaften (Berlin), Seite 142152., 1917, pp 142-152

Einstein A., de Sitter W., On the Relation between the Expansion and the Mean Density of the Universe, Proceedings of the National Academy of Science, 1932, vol. 18, p. 213

Faber S. M., Jackson R. E., Velocity dispersions and mass-to-light ratios for elliptical galaxies, ApJ, 1976, vol. 204, p. 668

Fan Y.-Z., Piran T., High-energy $\gamma$-ray emission from gamma-ray bursts - before GLAST, Frontiers of Physics in China, 2008, vol. 3, p. 306 
Foster J., Nightingale J. D., A Short Course in General Relativity. A Short Course in General Relativity, IX, 230 pp. 45 figs.. Springer-Verlag Berlin Heidelberg New York, 1995

Frail D. A., Kulkarni S. R., Nicastro L., Feroci M., Taylor G. B., The radio afterglow from the $\gamma$-ray burst of 8 May 1997, Nature, 1997, vol. 389, p. 261

Freedman W. L., Madore B. F., Gibson B. K., Ferrarese L., Kelson D. D., Sakai S., Mould J. R., Kennicutt Jr. R. C., Ford H. C., Graham J. A., Huchra J. P., Hughes S. M. G., Illingworth G. D., Macri L. M., Stetson P. B., Final Results from the Hubble Space Telescope Key Project to Measure the Hubble Constant, ApJ, 2001, vol. 553, p. 47

Freese K., Generalized cardassian expansion: a model in which the universe is flat, matter dominated, and accelerating, Nuclear Physics B Proceedings Supplements, 2003, vol. 124 , p. 50

Freese K., Cardassian expansion: dark energy density from modified Friedmann equations, New Astronomy Review, 2005, vol. 49, p. 103

Freese K., Lewis M., Cardassian expansion: a model in which the universe is flat, matter dominated, and accelerating, Physics Letters B, 2002, vol. 540, p. 1

Friedmann A., Über die Krümmung des Raumes, Zeitschrift für Physik, 1922, vol. 10, p. 377

Friedmann A., Über die Möglichkeit einer Welt mit konstanter negativer Krümmung des Raumes, Zeitschrift für Physik, 1924, vol. 21, p. 326

Fulling S. A., Parker L., Hu B. L., Conformal energy-momentum tensor in curved spacetime: Adiabatic regularization and renormalization, Phys. Rev. D, 1974, vol. 10, p. 3905

Giovi F., Amendola L., The distance-redshift equation in quintessence cosmology and the estimation of $H_{0}$ through time delays, MNRAS, 2001, vol. 325, p. 1097

Gondolo P., Freese K., Fluid interpretation of Cardassian expansion, Phys. Rev. D, 2003, vol. 68, p. 063509 
González-Díaz P. F., Sigüenza C. L., Phantom thermodynamics, Nuclear Physics B, 2004, vol. 697 , p. 363

Gron O., Repulsive gravitation and inflationary universe models., American Journal of Physics, 1986, vol. 54, p. 46

Gurvits L. I., Kellermann K. I., Frey S., The "angular size - redshift" relation for compact radio structures in quasars and radio galaxies, A\&A, 1999, vol. 342, p. 378

Hamuy M., Phillips M. M., Suntzeff N. B., Schommer R. A., Maza J., Aviles R., The Hubble Diagram of the Calan/Tololo Type IA Supernovae and the Value of HO, AJ, 1996, vol. 112, p. 2398

Hoyle F., Fowler W. A., Nucleosynthesis in Supernovae., ApJ, 1960, vol. 132, p. 565

Hu B. L., Parker L., Anisotropy damping through quantum effects in the early universe, Phys. Rev. D, 1978, vol. 17, p. 933

Hubble E., A Relation between Distance and Radial Velocity among Extra-Galactic Nebulae, Proceedings of the National Academy of Science, 1929, vol. 15, p. 168

Huterer D., Turner M. S., Probing dark energy: Methods and strategies, Phys. Rev. D, 2001, vol. 64, p. 123527

Isobe T., Feigelson E. D., Akritas M. G., Babu G. J., Linear regression in astronomy., ApJ, 1990, vol. 364, p. 104

Johri V. B., Parametrization of Dark Energy Equation of State, astro-ph/0409161, 2004

Kamenshchik A., Moschella U., Pasquier V., An alternative to quintessence, Physics Letters B, 2001, vol. 511, p. 265

Kantowski R., Corrections in the Luminosity-Redshift Relations of the Homogeneous FriedMann Models, ApJ, 1969, vol. 155, p. 89

Kellermann K. I., The cosmological deceleration parameter estimated from the angularsize/redshift relation for compact radio sources, Nature, 1993, vol. 361, p. 134 
Klebesadel R. W., Strong I. B., Olson R. A., Observations of Gamma-Ray Bursts of Cosmic Origin, ApJ, 1973, vol. 182, p. L85+

Kodama Y., Yonetoku D., Murakami T., Tanabe S., Tsutsui R., Nakamura T., Gammaray bursts in $1.8<z<5.6$ suggest that the time variation of the dark energy is small, MNRAS, 2008, vol. 391, p. L1

Komatsu E., Dunkley J., Nolta M. R., Bennett C. L., Gold B., Hinshaw G., Jarosik N., Larson D., Limon M., Page L., Spergel D. N., Halpern M., Hill R. S., Kogut A., Meyer S. S., Tucker G. S., Weiland J. L., Wollack E., Wright E. L., Five-Year Wilkinson Microwave Anisotropy Probe (WMAP) Observations: Cosmological Interpretation, ArXiv e-prints, 2008

Kowalski M., Rubin D., Aldering G., et al. Improved Cosmological Constraints from New, Old, and Combined Supernova Data Sets, ApJ, 2008, vol. 686, p. 749

Krauss L. M., Old Galaxies at High Redshift and the Cosmological Constant, ApJ, 1997, vol. 480 , p. 466

Kristian J., Sachs R. K., Observations in Cosmology, ApJ, 1966, vol. 143, p. 379

Landau L. D., Lifshitz E. M., The classical theory of fields. Course of theoretical physics Pergamon International Library of Science, Technology, Engineering and Social Studies, Oxford: Pergamon Press, 1971, 3rd rev. engl. edition, 1971

Lemaître G., Un Univers homogène de masse constante et de rayon croissant rendant compte de la vitesse radiale des nébuleuses extra-galactiques, Annales de la Societé Scientifique de Bruxelles, 1927, vol. 47, p. 49

Lemaitre G., Evolution of the Expanding Universe, Proceedings of the National Academy of Science, 1934, vol. 20, p. 12

Levinson A., Eichler D., The Difference between the Amati and Ghirlanda Relations, ApJ, 2005, vol. 629, p. L13

Lewis G. F., Ibata R. A., An investigation of gravitational lens determinations of $H_{0}$ in quintessence cosmologies, MNRAS, 2002, vol. 337, p. 26 
Lima J. A., Cunha J. V., Alcaniz J. S., Constraining the dark energy with galaxy cluster x-ray data, Phys. Rev. D, 2003, vol. 68, p. 023510

Lima J. A. S., Thermodynamics of decaying vacuum cosmologies, Phys. Rev. D, 1996, vol. 54 , p. 2571

Lima J. A. S., Cosmologies with Photon Creation and the 3K Relic Radiation Spectrum, General Relativity and Gravitation, 1997, vol. 29, p. 805

Lima J. A. S., Alternative Dark Energy Models: An Overview, Brazilian Journal of Physics, 2004, vol. 34, p. 194

Lima J. A. S., Abramo L. R. W., Deflation and matter creation., Physics Letters A, 1999, vol. 257, p. 123

Lima J. A. S., Alcaniz J. S., Angular size in "quintessence" cosmology, A\&A, 2000a, vol. 357, p. 393

Lima J. A. S., Alcaniz J. S., On the Minimal Angular Size in Open, $\Lambda$ CDM, and Scalar Field Cosmologies, General Relativity and Gravitation, 2000b, vol. 32, p. 1851

Lima J. A. S., Alcaniz J. S., Dark Energy and the Angular Size-Redshift Diagram for Milliarcsecond Radio Sources, ApJ, 2002, vol. 566, p. 15

Lima J. A. S., Alcaniz J. S., Thermodynamics, spectral distribution and the nature of dark energy, Physics Letters B, 2004, vol. 600, p. 191

Lima J. A. S., Alcaniz J. S., Cunha J. V., A simplified approach for Chaplygin-type cosmologies, Astroparticle Physics, 2008, vol. 30, p. 196

Lima J. A. S., Calvao M. O., Waga I., Cosmology, Thermodynamics and Matter Creation in: Frontier Physics, Essays in Honor of Jayme Tiomno. World Scientific, 1991

Lima J. A. S., Cunha J. V., Alcaniz J. S., Simplified Quartessence Cosmology, astro$\mathrm{ph} / 0611007,2006$

Lima J. A. S., Germano A. S. M., On the equivalence of bulk viscosity and matter creation., Physics Letters A, 1992, vol. 170, p. 373 
Lima J. A. S., Germano A. S. M., Abramo L. R. W., FRW-type cosmologies with adiabatic matter creation, Phys. Rev. D, 1996, vol. 53, p. 4287

Lima J. A. S., Jesus J. F., Cunha J. V., Can Old Galaxies at High Redshifts and Baryon Acoustic Oscillations Constrain $H_{0}$ ?, ApJ, 2009, vol. 690, p. L85

Lima J. A. S., Maia Jr. A., Thermodynamic properties of $\gamma$ fluids and the quantum vacuum, Phys. Rev. D, 1995a, vol. 52, p. 5628

Lima J. A. S., Maia Jr. A., Thermodynamic properties of $\gamma$ fluids and the quantum vacuum, Phys. Rev. D, 1995b, vol. 52, p. 5628

Lima J. A. S., Maia J. M. F., Deflationary cosmology with decaying vacuum energy density, Phys. Rev. D, 1994, vol. 49, p. 5597

Lima J. A. S., Pereira S. H., Chemical potential and the nature of dark energy: The case of a phantom field, Phys. Rev. D, 2008, vol. 78, p. 083504

Lima J. A. S., Silva F. E., Santos R. C., Accelerating cold dark matter cosmology $\left(\Omega_{\Lambda} \equiv 0\right)$, Classical and Quantum Gravity, 2008, vol. 25, p. 205006

Lima J. A. S., Trodden M., Decaying vacuum energy and deflationary cosmology in open and closed universes, Phys. Rev. D, 1996, vol. 53, p. 4280

Linder E. V., Light propagation in generalized Friedmann universes, A\&A, 1988, vol. 206, p. 190

Linder E. V., Exploring the Expansion History of the Universe, Physical Review Letters, 2003, vol. 90, p. 091301

Maia J. M., Lima J. A., Scalar field description of decaying- $\Lambda$ cosmologies, Phys. Rev. D, 2002, vol. 65, p. 083513

Maia J. M. F., Lima J. A. S., Extended warm inflation, Phys. Rev. D, 1999, vol. 60, p. 101301

Metzger M. R., Djorgovski S. G., Kulkarni S. R., et al. Spectral constraints on the redshift of the optical counterpart to the $\gamma$-ray burst of 8 May 1997, Nature, 1997, vol. 387, p. 878 
Mosquera Cuesta H. J., Dumet M H., Furlanetto C., Confronting the Hubble diagram of gamma-ray bursts with Cardassian cosmology, Journal of Cosmology and Astro-Particle Physics, 2008, vol. 7, p. 4

Overduin J. M., Cooperstock F. I., Evolution of the scale factor with a variable cosmological term, Phys. Rev. D, 1998, vol. 58, p. 043506

Ozer M., Taha M. O., A possible solution to the main cosmological problems., Physics Letters B, 1986, vol. 171, p. 363

Ozer M., Taha M. O., A model of the universe free of cosmological problems., Nuclear Physics B, 1987, vol. 287, p. 776

Papastamatiou N. J., Parker L., Asymmetric creation of matter and antimatter in the expanding universe., Phys. Rev. D, 1979, vol. 19, p. 2283

Parker L., Particle Creation in Expanding Universes, Physical Review Letters, 1968, vol. 21, p. 562

Parker L., Quantized fields and particle creation in expanding universes. I., Physical Review, 1969, vol. 183, p. 1057

Peebles P. J. E., Principles of physical cosmology. Princeton Series in Physics, Princeton, NJ: Princeton University Press, — c1993, 1993

Penzias A. A., Wilson R. W., A Measurement of Excess Antenna Temperature at 4080 Mc/s., ApJ, 1965, vol. 142, p. 419

Pereira S. H., Lima J. A. S., On phantom thermodynamics, Physics Letters B, 2008, vol. 669 , p. 266

Perlmutter S. e. a., Measurements of Omega and Lambda from 42 High-Redshift Supernovae, ApJ, 1999, vol. 517, p. 565

Phillips M. M., The absolute magnitudes of Type IA supernovae, ApJ, 1993, vol. 413, p. L105

Piran T., Astronomy: Glowing embers, Nature, 2003, vol. 422, p. 268 
Piran T., The physics of gamma-ray bursts, Reviews of Modern Physics, 2004, vol. 76, p. 1143

Pires N., Zhu Z.-H., Alcaniz J. S., Lookback time as a test for brane cosmology, Phys. Rev. D, 2006, vol. 73, p. 123530

Prigogine I., Geheniau J., Gunzig E., Nardone P., Thermodynamics and cosmology, General Relativity and Gravitation, 1989, vol. 21, p. 767

Randall L., Sundrum R., An Alternative to Compactification, Physical Review Letters, 1999a, vol. 83, p. 4690

Randall L., Sundrum R., Large Mass Hierarchy from a Small Extra Dimension, Physical Review Letters, 1999b, vol. 83, p. 3370

Ratra B., Peebles P. J. E., Cosmological consequences of a rolling homogeneous scalar field, Phys. Rev. D, 1988, vol. 37, p. 3406

Rees M. J., Mészáros P., Dissipative Photosphere Models of Gamma-Ray Bursts and X-Ray Flashes, ApJ, 2005, vol. 628, p. 847

Riess A. G., Filippenko A. V., Challis P., et al. Observational Evidence from Supernovae for an Accelerating Universe and a Cosmological Constant, AJ, 1998, vol. 116, p. 1009

Riess A. G., Strolger L.-G., Casertano S., et al. New Hubble Space Telescope Discoveries of Type Ia Supernovae at $z \geq 1$ : Narrowing Constraints on the Early Behavior of Dark Energy, ApJ, 2007, vol. 659, p. 98

Robertson H. P., Kinematics and World-Structure II., ApJ, 1936, vol. 83, p. 187

Ruffini R., Bianco C. L., Chardonnet P., Fraschetti F., Vitagliano L., Xue S.-S., New perspectives in physics and astrophysics from the theoretical understanding of GammaRay Bursts. In Cosmology and Gravitation, vol. 668 of American Institute of Physics Conference Series, 2003, p. 16

Sachs R., Gravitational Waves in General Relativity. VI. The Outgoing Radiation Condition, Royal Society of London Proceedings Series A, 1961, vol. 264, p. 309 
Santos R. C., Efeitos das Inomogeneidades da Matéria em Cosmologias Aceleradas, Universidade Federal do Rio Grande do Norte, 2007, Tese de Doutorado, 198 p.

Santos R. C., Cunha J. V., Lima J. A. S., Constraining the dark energy and smoothness parameter with supernovae, Phys. Rev. D, 2008, vol. 77, p. 023519

Santos R. C., Lima J. A. S., Clustering, angular size, and dark energy, Phys. Rev. D, 2008, vol. 77 , p. 083505

Schaefer B. E., The Hubble Diagram to Redshift greater than 6 from 69 Gamma-Ray Bursts, ApJ, 2007, vol. 660, p. 16

Schneider P., Ehlers J., Falco E. E., Gravitational Lenses. Gravitational Lenses, XIV, 560 pp. 112 figs.. Springer-Verlag Berlin Heidelberg New York. Also Astronomy and Astrophysics Library, 1992

Sereno M., Piedipalumbo E., Sazhin M. V., Effects of quintessence on observations of Type Ia supernovae in the clumpy universe, MNRAS, 2002, vol. 335, p. 1061

Spergel D. N., Bean R., Doré O., Nolta M. R., Bennett C. L., et al. Three-Year Wilkinson Microwave Anisotropy Probe (WMAP) Observations: Implications for Cosmology, ApJS, 2007, vol. 170, p. 377

Steigman G., Santos R. C., Lima J. A. S., An Accelerating Cosmology Without Dark Energy, astro-ph/0812.3912, 2008

Tegmark M., Blanton M. R., Strauss M. A., Hoyle F., et al. The Three-Dimensional Power Spectrum of Galaxies from the Sloan Digital Sky Survey, ApJ, 2004, vol. 606, p. 702

Trotta R., Bayes in the sky: Bayesian inference and model selection in cosmology, Contemporary Physics, 2008, vol. 49, p. 71

Tully R. B., Fisher J. R., A new method of determining distances to galaxies, A\&A, 1977, vol. 54 , p. 661

Turner M. S., White M., CDM models with a smooth component, Phys. Rev. D, 1997, vol. 56, p. R4439 
van Paradijs J., Groot P. J., Galama T., et al. Transient optical emission from the error box of the $\gamma$-ray burst of 28 February 1997, Nature, 1997, vol. 386, p. 686

Walker A. G., On Milne's theory of world structure, Proc. Lond. Math. Soc., 1936, vol. 42, p. 90

Wang F. Y., Constraints on the Cardassian expansion models from the latest observational data, Journal of Cosmology and Astro-Particle Physics, 2007, vol. 8, p. 20

Wang P., Meng X.-H., Can vacuum decay in our universe?, Classical and Quantum Gravity, 2005, vol. 22, p. 283

Weinberg S., Gravitation and Cosmology: Principles and Applications of the General Theory of Relativity. John Wiley \& Sons, 1972

Weinberg S., The cosmological constant problem, Reviews of Modern Physics, 1989, vol. 61, p. 1

Weinberg S., Cosmology. Oxford University Press, 2008

Weller J., Albrecht A., Future supernovae observations as a probe of dark energy, Phys. Rev. D, 2002, vol. 65, p. 103512

Yamazaki R., Ioka K., Nakamura T., Peak Energy-Isotropic Energy Relation in the OffAxis Gamma-Ray Burst Model, ApJ, 2004, vol. 606, p. L33

Yonetoku D., Murakami T., Nakamura T., Yamazaki R., Inoue A. K., Ioka K., GammaRay Burst Formation Rate Inferred from the Spectral Peak Energy-Peak Luminosity Relation, ApJ, 2004, vol. 609, p. 935

Zeldovich Y. B., Observations in a Universe Homogeneous in the Mean, Soviet Astronomy, 1964, vol. 8, p. 13

Zhu Z.-H., Fujimoto M.-K., Cardassian Expansion: Constraints from Compact Radio Source Angular Size versus Redshift Data, ApJ, 2002, vol. 581, p. 1

Zimdahl W., Schwarz D. J., Balakin A. B., Pavón D., Cosmic antifriction and accelerated expansion, Phys. Rev. D, 2001, vol. 64, p. 063501 
Apêndice 

Apêndice A

\section{Equação do Desvio Geodésico}

Neste apêndice deduzimos a equação do desvio geodésico, que é a equação de partida para a dedução da equação óptica de Sachs (4.24). Vamos apresentar aqui a dedução proposta por Foster e Nightingale (1995).

Considere duas geodésicas vizinhas parametrizadas por $\lambda: x^{\alpha}(\lambda)$ e $\tilde{x}^{\alpha}(\lambda)$. Seja $\xi^{\alpha}(\lambda)$ o vetor que liga as duas geodésicas para o mesmo parâmetro afim $\lambda$, ou seja, $\xi^{\alpha}(\lambda)=$ $\tilde{x}^{\alpha}(\lambda)-x^{\alpha}(\lambda)$. O parâmetro afim é definido de tal forma que $\xi^{\alpha}(\lambda)$ seja um pequeno vetor deslocamento, isto é, que realmente descrevamos geodésicas vizinhas.

Visto que $x^{\alpha}(\lambda)$ e $\tilde{x}^{\alpha}(\lambda)$ são geodésicas, tais quantidades satisfazem (Landau e Lifshitz, 1971):

$$
\frac{d^{2} x^{\alpha}}{d \lambda^{2}}+\Gamma_{\beta \gamma}^{\alpha} \frac{d x^{\beta}}{d \lambda} \frac{d x^{\gamma}}{d \lambda}=0
$$

$\mathrm{e}$

$$
\frac{d^{2} \tilde{x}^{\alpha}}{d \lambda^{2}}+\tilde{\Gamma}_{\beta \gamma}^{\alpha} \frac{d \tilde{x}^{\beta}}{d \lambda} \frac{d \tilde{x}^{\gamma}}{d \lambda}=0
$$

onde $\Gamma_{\beta \gamma}^{\alpha}$ são os símbolos de Christoffel e o til indica que os valores são tomados nas coordenadas $\tilde{x}^{\alpha}(\lambda)$. Expandindo $\tilde{\Gamma}_{\beta \gamma}^{\alpha}$ em primeira ordem em $\xi^{\alpha}(\lambda)$ temos que:

$$
\tilde{\Gamma}_{\beta \gamma}^{\alpha}=\Gamma_{\beta \gamma}^{\alpha}+\Gamma_{\beta \gamma, \delta}^{\alpha} \xi^{\delta}
$$

Subtraindo a equação (A.1) da equação (A.2) e considerando apenas termos de primeira ordem temos

$$
\ddot{\xi}^{\alpha}+\Gamma_{\beta \gamma, \delta}^{\alpha} \dot{x}^{\beta} \dot{x}^{\gamma} \xi^{\delta}+\Gamma_{\beta \gamma}^{\alpha} \dot{x}^{\beta} \dot{\xi}^{\gamma}+\Gamma_{\beta \gamma}^{\alpha} \dot{\xi}^{\beta} \dot{x}^{\gamma}=0,
$$


onde o ponto significa derivação com relação ao parâmetro afim $\lambda$. Note que a equação acima pode ser reescrita da seguinte forma:

$$
\frac{d\left(\dot{\xi}^{\alpha}+\Gamma_{\beta \gamma}^{\alpha} \xi^{\beta} \dot{x}^{\gamma}\right)}{d \lambda}-\Gamma_{\beta \gamma, \delta}^{\alpha} \xi^{\beta} \dot{x}^{\gamma} \dot{x}^{\delta}-\Gamma_{\beta \gamma}^{\alpha} \xi^{\beta} \ddot{x}^{\gamma}+\Gamma_{\beta \gamma, \delta}^{\alpha} \dot{x}^{\beta} \dot{x}^{\gamma} \xi^{\delta}+\Gamma_{\beta \gamma}^{\alpha} \dot{x}^{\beta} \dot{\xi}^{\gamma}=0
$$

Utilizando a equação (A.1) para $\ddot{x}^{\gamma}$ e rearranjando alguns índices mudos obtemos

$$
\xi_{; \beta \gamma}^{\alpha} \dot{x}^{\beta} \dot{x}^{\gamma}+\left(\Gamma_{\gamma \delta, \beta}^{\alpha}-\Gamma_{\beta \gamma, \delta}^{\alpha}+\Gamma_{\beta \epsilon}^{\alpha} \Gamma_{\delta \gamma}^{\epsilon}-\Gamma_{\epsilon \delta}^{\alpha} \Gamma_{\beta \gamma}^{\epsilon}\right) \xi^{\beta} \dot{x}^{\gamma} \dot{x}^{\delta}=0
$$

Finalmente, pela definição do tensor de Riemann $R_{\beta \gamma \delta}^{\alpha}$

$$
R_{\beta \gamma \delta}^{\alpha}=-\Gamma_{\gamma \delta, \beta}^{\alpha}+\Gamma_{\beta \gamma, \delta}^{\alpha}-\Gamma_{\beta \epsilon}^{\alpha} \Gamma_{\delta \gamma}^{\epsilon}+\Gamma_{\epsilon \delta}^{\alpha} \Gamma_{\beta \gamma}^{\epsilon}
$$

obtemos de (A.6)

$$
\xi_{; \beta \gamma}^{\alpha} \dot{x}^{\beta} \dot{x}^{\gamma}=R_{\beta \gamma \delta}^{\alpha} \xi^{\beta} \dot{x}^{\gamma} \dot{x}^{\delta},
$$

que é a equação do desvio geodésico. 
Apêndice B

\section{Influência dos Priors sobre $H_{0}$ nos Vínculos Cosmológicos}

Neste apêndice discutimos a influência dos priors sobre $H_{0}$ quando vinculamos parâmetros cosmológicos. Utilizamos aqui dois priors bastante discutidos na literatura, a saber, considerar $H_{0}$ fixo (prior delta de Dirac) e um prior flat. O objetivo é enfatizar que o espaço de parâmetros resultante é dependente do prior adotado. Além disso, como a calibração realizada para os gamma-ray bursts depende da cosmologia, é interessante verificarmos se o prior assumido leva a resultados que independem do modelo cosmológico utilizado para a calibração.

\section{B.1 $H_{0}$ fixo}

Neste caso, vamos considerar o valor de $H_{0}$ fornecido pelo Hubble Space Telescope (Freedman et al., 2001), dado por $72 \mathrm{Km} \mathrm{s}^{-1} \mathrm{Mpc}^{-1}$. Fixar o valor de $H_{0}$ significa considerar um prior do tipo delta de Dirac: $\pi\left(H_{0}\right)=\delta\left(H_{0}-72\right)$. Neste caso a integral (5.6) é trivial e o $\chi^{2}$ nesse caso é dado por:

$$
\chi^{2}=\sum_{i}^{N} \frac{\left(\mu_{\text {teor }}\left(z_{i}, \alpha, \Omega_{M}, H_{0}=72\right)-\mu_{i}\left(z_{i}\right)\right)^{2}}{\sigma_{\mu_{i}}^{2}} .
$$

Os resultados obtidos dessa abordagem serão discutidos nas próximas subseções.

\section{B.1.1 Supernovas}

Realizamos o teste $\chi^{2}$ para a amostra de 307 supernovas de Kowalski et al. (2008), a fim de vincular os parâmetros $\alpha$ e $\Omega_{M}$, onde os resultados obtidos estão mostrados na 
figura B.1. Vemos que a imposição de fixar o parâmetro de Hubble restringe bem o valor de $\Omega_{M}$, sendo consistente com o encontrado na literatura, mas não conseguimos obter o mesmo vínculo para $\alpha$. Mesmo considerando um vínculo mais fraco para o parâmetro de aglomeramento $\alpha$, é importante notar que conseguimos restringi-lo em um nível de significância de $99.7 \%$ para $\alpha>0.4$. Portanto, vemos que a hipótese de um $H_{0}$ fixo exclui a possibilidade de um modelo onde toda a matéria está aglomerada, mas é compatível com um modelo onde toda a matéria está distribuída de forma homogênea.
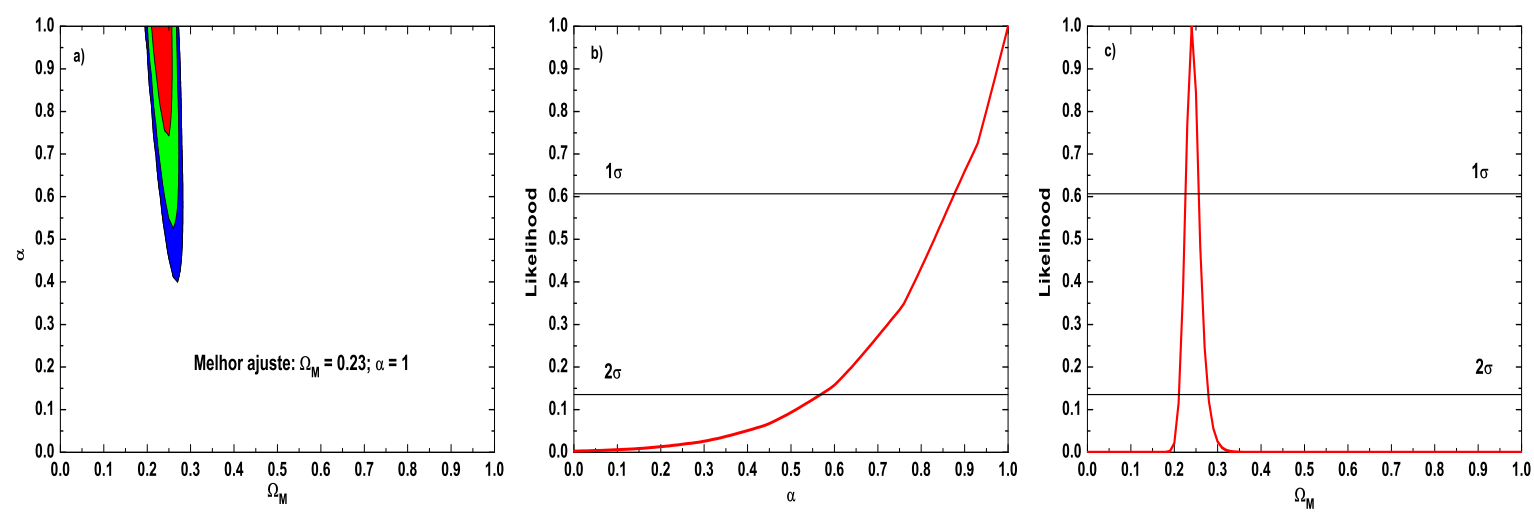

Figura B.1: a) Plano $\Omega_{M}-\alpha$ para o modelo $\Lambda$ CDM plano obtido de 307 dados de supernovas. Os contornos representam níveis de significância de $68.3 \%, 95.4 \%$ e $99.7 \%$ respectivamente. b) Likelihood para o parâmetro de aglomeramento $\alpha$. Com um nível de significância de $95.4 \%$ obtivemos o intervalo $0.57 \leq \alpha \leq 1$. c) Likelihood para o parâmetro de densidade da matéria $\Omega_{M}$. Neste caso obtivemos $0.21 \leq \Omega_{M} \leq 0.27$ com $95.4 \%$ de confiança estatística.

\section{B.1.2 Gamma-Ray Bursts}

Uma análise com gamma-ray bursts foi feita utilizando os 69 GRBs calibrados por Schaefer (2007). Os resultados obtidos pelo teste $\chi^{2}$ estão apresentados na figura B.2. Para os dados de GRBs, o melhor ajuste encontrado foi $\Omega_{M}=0.29$ e $\alpha=1$, portanto, com vínculos mais fracos do que obtidos com supernovas. Vemos que o melhor ajuste está de acordo com o obtido na literatura, mas estatisticamente o resultado não exclui valores baixos de $\alpha$, com $\alpha>0.4$ dentro de $2 \sigma$ de confiança. O vínculo para $\Omega_{M}$ foi semelhante ao obtido com supernovas.

Fizemos também o teste $\chi^{2}$ para a amostra de 69 gamma-ray bursts calibrados para o modelo cardassiano (Mosquera Cuesta et al., 2008). Os resultados obtidos são mostrados na figura B.3. Vemos que o melhor ajuste foi deslocado para um valor de $\Omega_{M}$ maior, igual 

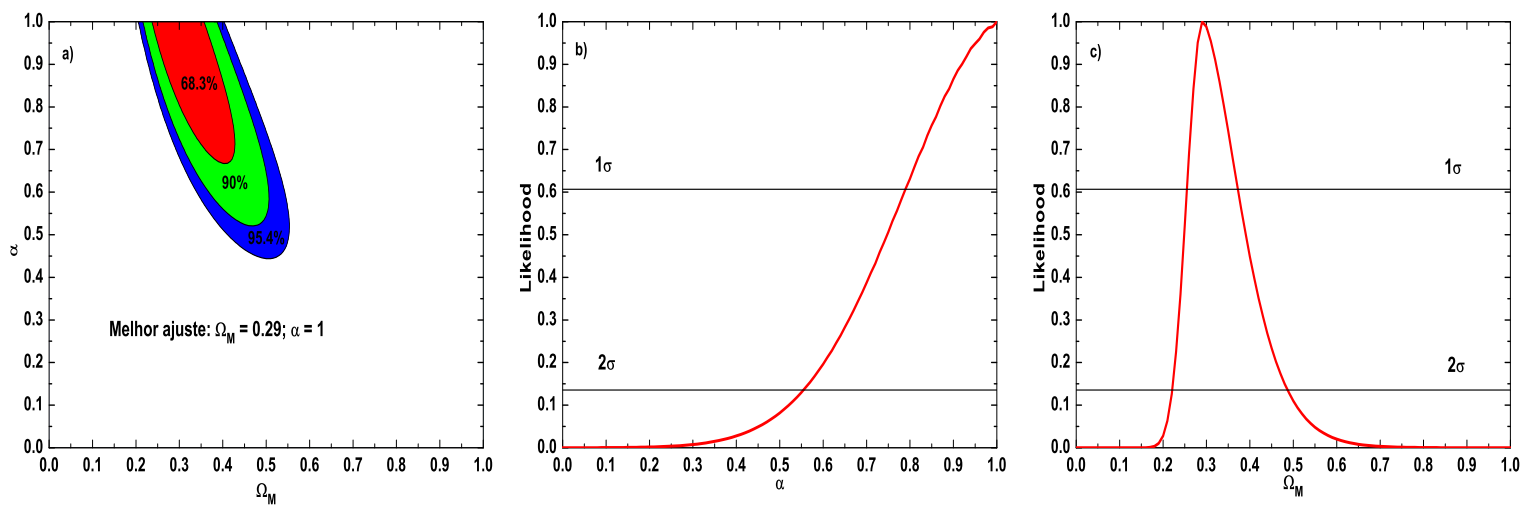

Figura B.2: a) Plano $\Omega_{M}-\alpha$ para o modelo $\Lambda$ CDM obtido de 69 GRBs utilizando a calibração de Schaefer. Os níveis de confiança estatística são os indicados na figura. b) Likelihood para o parâmetro de aglomeramento $\alpha$, onde dentro de um nível de significância de $95.4 \%$ obtivemos $0.34 \leq \alpha \leq 1$. c) Likelihood para o parâmetro de densidade da matéria $\Omega_{M}$. Para tal parâmetro obtivemos dentro de um nível de significância de $95.4 \%$ o intervalo $0.23 \leq \Omega_{M} \leq 0.48$.

a 0.39 , e para um valor de $\alpha$ diferente de 1 , igual a 0.94 . Logo, vemos que uma calibração diferente pode levar a resultados que, embora compatíveis, dão uma diferença de relativa de $25 \%$ no melhor ajuste para $\Omega_{M}$. Também devemos levar em consideração que a hipótese de um $H_{0}$ fixo é muito restritiva, e que hipóteses mais realistas podem levar a resultados que reflitam melhor os vínculos observacionais frente às observações atuais.
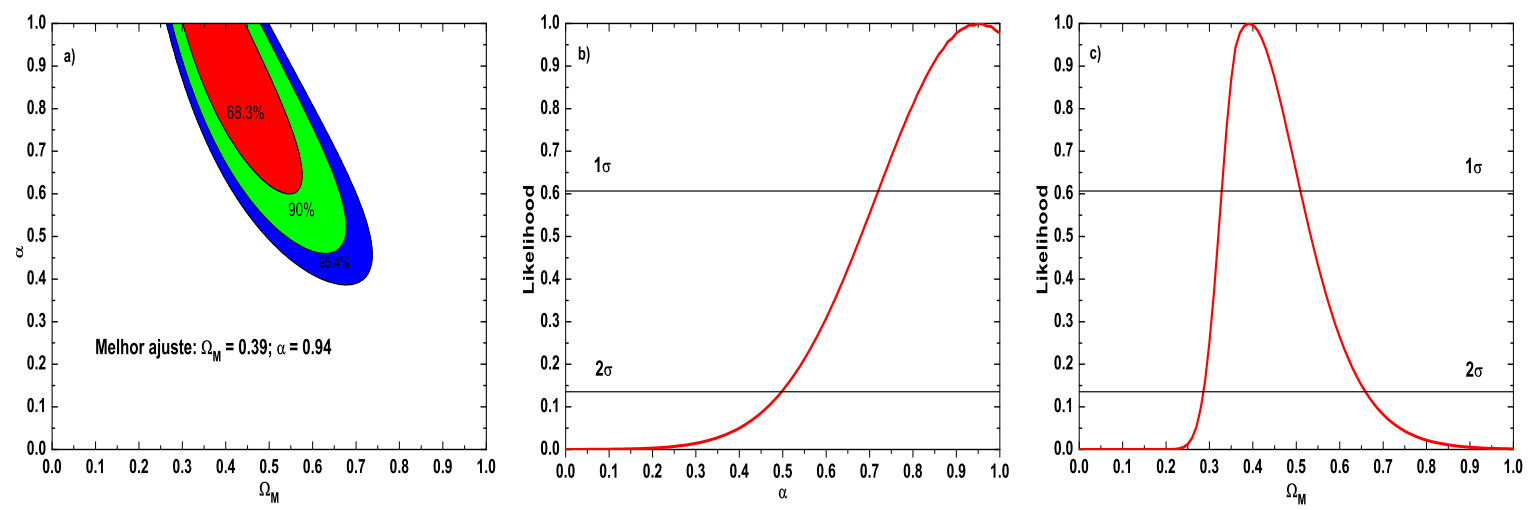

Figura B.3: a) Contornos no plano $\Omega_{M}-\alpha$ para o modelo $\Lambda$ CDM obtido de 69 GRBs utilizando a calibração para o modelo cardassiano. b) Likelihood para o parâmetro de aglomeramento $\alpha$. Obtivemos dentro de um nível de significância de $95.4 \%$ o intervalo $0.5 \leq \alpha \leq 1$. c) Likelihood para $\Omega_{M}$. Com $95.4 \%$ de confiança estatística, o parâmetro de densidade da matéria está limitado no intervalo $0.29 \leq \Omega_{M} \leq 0.65$. 


\section{B.1.3 Supernovas e Gamma-Ray Bursts}

Uma vez estudadas as amostras de supernovas de Kowalski et al. (2008) e gamma-ray bursts (Schaefer, 2007; Mosquera Cuesta et al., 2008) separadamente, é de grande interesse uma análise conjunta considerando as duas amostras. Por isto, devemos considerar cada calibração separadamente. Primeiramente, estudaremos as 307 supernovas de Kowalski et al. (2008) (Union Compilation Data) e os 69 GRBs calibrados por Schaefer (2007). Os parâmetros $\alpha$ e $\Omega_{M}$ foram vinculados pelo teste $\chi^{2}$, sendo que para a análise conjunta é suficiente somar em quadratura os valores de cada amostra: $\chi^{2}=\chi_{S N}^{2}+\chi_{G R B}^{2}$.

Os resultados obtidos estão apresentados na figura B.4. Vemos que ambos os parâmetros são bem vinculados. Neste caso, $\alpha>0.6$ com um nível de confiança de $99.7 \%$ e $\alpha>0.86$ com um nível de confiança de $95.4 \%$. Tal análise mostra que a combinação das amostras possibilitou a quebra da degenerescência entre os parâmetros, sendo o valor de $\Omega_{M}$ compatível com análises independentes.
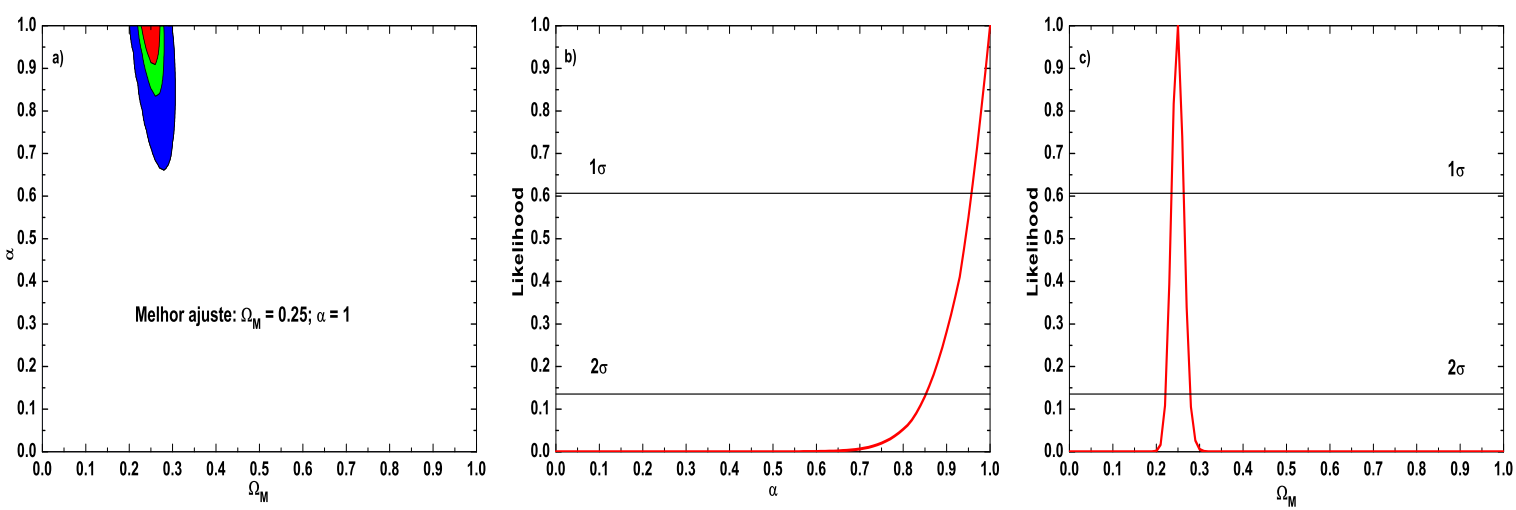

Figura B.4: a) Plano $\Omega_{M}-\alpha$ para o modelo $\Lambda$ CDM plano obtido de 307 dados de supernovas e 69 GRBs utilizando a calibração de Schaefer. Os contornos representam níveis de confiança estatística de $68.3 \%, 95.4 \%$ e $99.7 \%$, respectivamente. b) Likelihood para o parâmetro de aglomeramento $\alpha$. Para esse parâmetro obtivemos dentro de um nível de significância de $95.4 \%$ o intervalo $0.86 \leq \alpha \leq 1$. c) Likelihood para o parâmetro de densidade da matéria $\Omega_{M}$, onde obtivemos dentro de um nível de significância de $95.4 \%$ o intervalo $0.23 \leq \Omega_{M} \leq 0.27$.

Para as 307 supernovas da amostra de Kowalski et al. (2008) (Union Compilation Data) e os 69 GRBs calibrados para o modelo cardassiano (Mosquera Cuesta et al., 2008); os resultados obtidos estão apresentados na figura B.5. Vemos que ambas as calibrações apresentam o mesmo melhor ajuste: $\Omega_{M}=0.25$ e $\alpha=1$, mas no último caso o parâmetro de aglomeramento é melhor vinculado dentro de um nível de significância de $99.7 \%$, com 
$\alpha>0.8$.
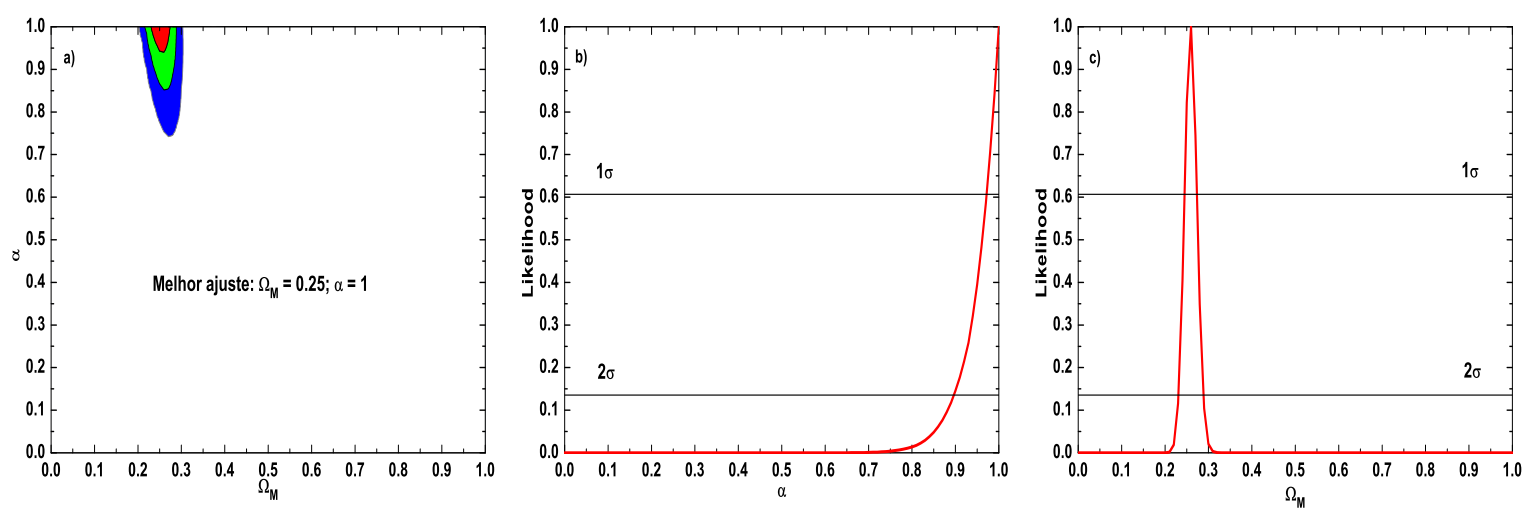

Figura B.5: a) Contornos no plano $\Omega_{M}-\alpha$ para o modelo $\Lambda$ CDM plano obtido de 307 dados de supernovas e 69 GRBs utilizando a calibração para o modelo cardassiano, que representam níveis de significância estatística de $68.3 \%, 95.4 \%$ e 99.7\%, respectivamente. b) Likelihood para o parâmetro de aglomeramento $\alpha$. Dentro de um nível de significância de $95.4 \%$ obtivemos o intervalo $0.9 \leq \alpha \leq 1$. c) Likelihood para o parâmetro de densidade da matéria $\Omega_{M}$. Para esse parâmetro obtivemos dentro de um nível de significância de $95.4 \%$ o intervalo $0.24 \leq \Omega_{M} \leq 0.28$.

Na tabela B.1 apresentamos os resultados obtidos com as amostras de supernovas de Kowalski et al. (2008) e GRBs calibrados por Schaefer (2007) e para o modelo cardassiano (Mosquera Cuesta et al., 2008). Isoladamente as amostras apresentaram vínculos parecidos aos parâmetros, com a diferença que supernovas vinculam bem $\Omega_{M}$. Já quando as amostras são combinadas, percebemos que ambos os parâmetros são bem vinculados, o que demonstra a importância da análise conjunta em quebrar a degenerescência entre os parâmetros. Com relação às calibrações adotadas, vemos que embora os resultados obtidos sejam semelhantes, os vínculos apresentaram diferenças em alguns aspectos. O espaço de parâmetros com a calibração para o modelo cardassiano foi melhor vinculado do que com a calibração de Schaefer. Isso pode ser entendido pela hipótese para o parâmetro de Hubble ser mais restritiva e a calibração ser dependente do valor de $H_{0}$. Portanto, para que os vínculos reflitam de forma confiável os dados observacionais, devemos tomar um prior condizente com nosso conhecimento prévio de $H_{0}$. 
Tabela B.1 - Vínculos para os parâmetros $\alpha$ e $\Omega_{M}$ considerando $H_{0}$ fixo. $\chi_{\min }^{2}$ representa o $\chi^{2}$ mínimo obtido, o subscrito $M A$ representa o melhor ajuste obtido e $2 \sigma$ o intervalo de confiança estatística de 95.4\%. (S) representa os GRBs calibrados por Schaefer (2007) e (C) os GRBs calibrados para o modelo cardassiano (Mosquera Cuesta et al., 2008).

\begin{tabular}{lccccc}
\hline \hline \multicolumn{1}{c}{ Amostra } & $\chi_{\text {min }}^{2}$ & $\alpha_{M A}$ & $\left(\Omega_{M}\right)_{M A}$ & $2 \sigma$ & $2 \sigma$ \\
\hline SN & 323.48 & 1 & 0.23 & $0.57 \leq \alpha \leq 1.0$ & $0.21 \leq \Omega_{M} \leq 0.27$ \\
GRB(S) & 71.25 & 1 & 0.29 & $0.34 \leq \alpha \leq 1.0$ & $0.23 \leq \Omega_{M} \leq 0.48$ \\
GRB(C) & 71.36 & 0.94 & 0.39 & $0.50 \leq \alpha \leq 1.0$ & $0.29 \leq \Omega_{M} \leq 0.65$ \\
SN e GRB(S) & 391.45 & 1 & 0.25 & $0.86 \leq \alpha \leq 1.0$ & $0.23 \leq \Omega_{M} \leq 0.27$ \\
SN e GRB(C) & 404.22 & 1 & 0.25 & $0.90 \leq \alpha \leq 1.0$ & $0.24 \leq \Omega_{M} \leq 0.28$ \\
& & & & & \\
\hline
\end{tabular}

\section{B.2 Prior flat em $H_{0}$}

Para realizarmos o teste $\chi^{2}$ tomando um prior flat em $H_{0}$, devemos marginalizar sobre tal parâmetro considerando que todos valores para o parâmetro são igualmente prováveis, ou seja, tomamos um peso $\pi\left(H_{0}\right)=1$. Assim, o novo $\chi^{2}$ é dado por:

$$
\tilde{\chi^{2}}=-2 \ln \mathcal{L}
$$

onde a Likelihood $\mathcal{L}$ é marginalizada em $H_{0}$ :

$$
\mathcal{L}=\int e^{-\frac{\chi^{2}}{2}} d H_{0}
$$

Feita a marginalização podemos realizar o teste, cujos resultados são discutidos abaixo.

\section{B.2.1 Supernovas}

Vinculamos os parâmetros $\alpha$ e $\Omega_{M}$ para as 307 supernovas da amostra de Kowalski et al. (2008) (Union Compilation Data) através do teste $\chi^{2}$. Os resultados obtidos estão apresentados na figura B.6. Vemos que o parâmetro de densidade da matéria é bem vinculado, mas o parâmetro de aglomeramento é fracamente vinculado, $\alpha>0.1$ dentro de um nível de significância de 95.4\% . Ainda assim, o melhor ajuste privilegia um universamente perfeitamente homogêneo e isotrópico e com um parâmetro de densidade compatível com 
outros testes cosmológicos. Também podemos analisar que o resultado obtido comparado a supor $H_{0}$ fixo foi parecido, apenas com poder de restrição menor.
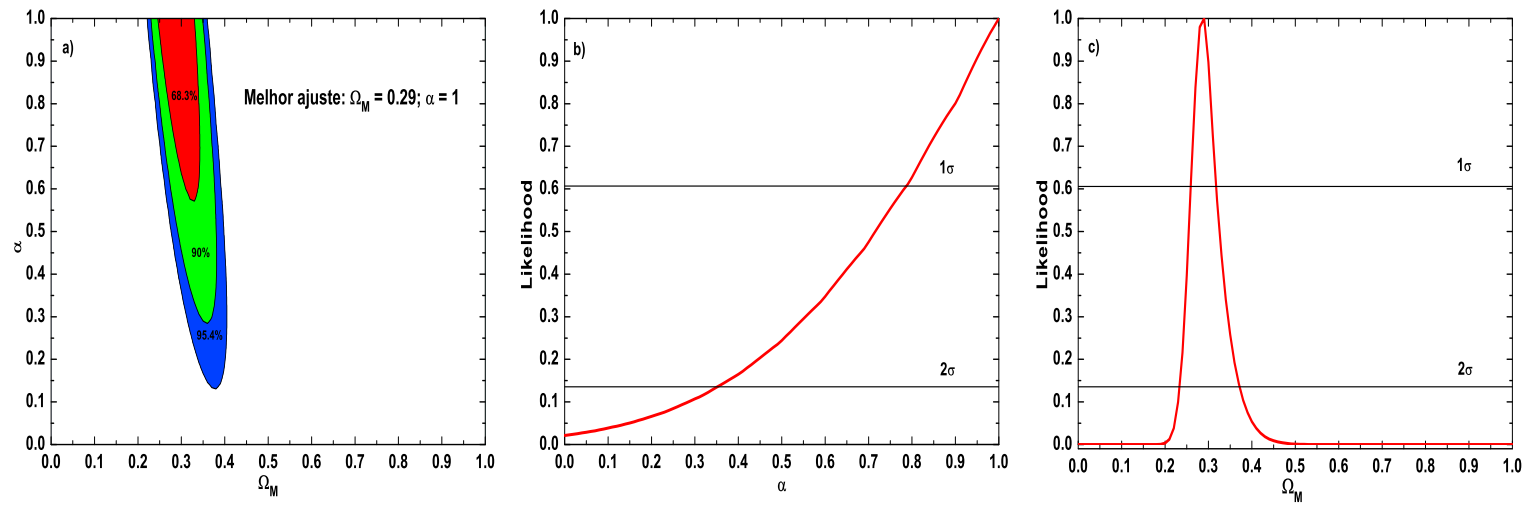

Figura B.6: a) Contornos no plano $\Omega_{M}-\alpha$ para o modelo $\Lambda$ CDM plano obtido de 307 dados de supernovas.

b) Likelihood para o parâmetro de aglomeramento $\alpha$. Dentro de um nível de significância de $95.4 \%$ obtivemos o intervalo $0.36 \leq \alpha \leq 1.0$. c) Likelihood para o parâmetro de densidade da matéria $\Omega_{M}$, onde obtivemos com um nível de significância de $95.4 \%$ o intervalo $0.24 \leq \Omega_{M} \leq 0.37$.

Podemos comparar esse resultado com o resultado obtido por Santos et al. (2008), que é mostrado na figura B.7. O melhor ajuste obtido foi de $\Omega_{M}=0.33$ e $\alpha=1$, sendo que $\alpha>0.42$ dentro de um nível de significância de 95.4\%. Vemos que a inclusão de mais dados fez com que o vínculo sobre o parâmetro de densidade da matéria seja melhor, enquanto o oposto ocorre para o parâmetro de aglomeramento. Esse fato pode ser entendido pela percepção de que dados em baixos redshifts não vinculam bem o parâmetro de aglomeramento, mas vinculam bem o parâmetro de densidade da matéria, como acontece com os dados da oscilação acústica dos bárions (Cole et al., 2005; Tegmark et al., 2004). De todo modo, o melhor ajuste ainda favorece um modelo homogêneo e isotrópico em todas as escalas.

\section{B.2.2 Gamma-Ray Bursts}

Consideremos agora a amostra de 69 gamma-ray bursts calibrados por Schaefer (2007). Tal amostra foi utilizada para vincular os parâmetros $\alpha$ e $\Omega_{M}$ pelo teste $\chi^{2}$. Os resultados obtidos são mostrados na figura B.8. Vemos que o parâmetro de densidade da matéria é fracamente vinculado nesse caso, o mesmo ocorrendo com o parâmetro de aglomeramento, onde $\alpha>0.35$ dentro de um nível de significância de 95.4\%. Vemos que o resultado depende fortemente da hipótese sobre $H_{0}$, uma vez que existe uma diferença drástica 

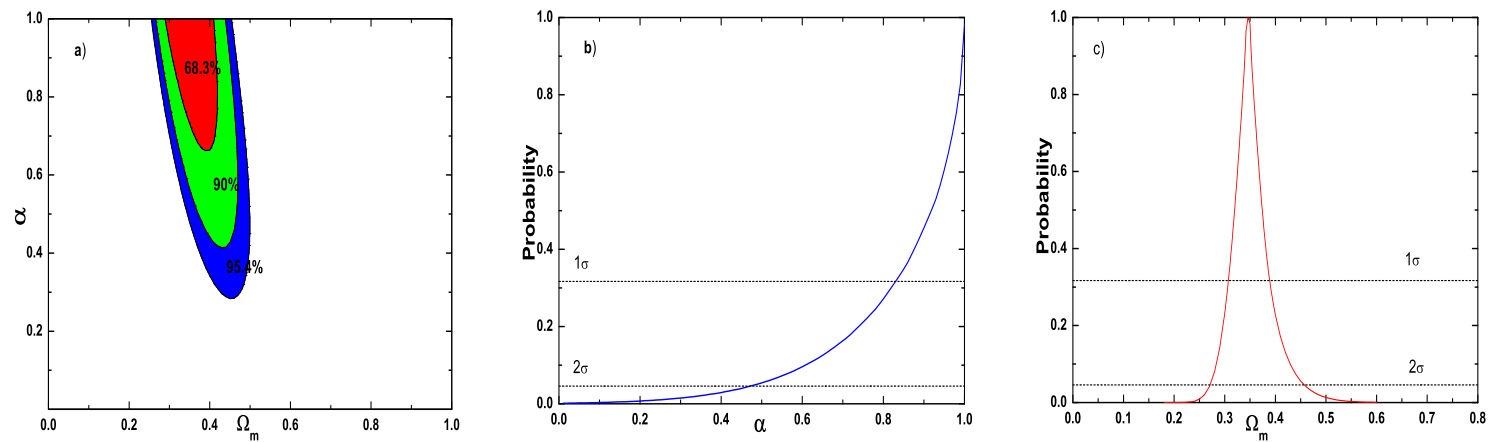

Figura B.7: Figura extraída de Santos et al. (2008). a) Contornos no plano $\Omega_{M}-\alpha$ para o modelo $\Lambda$ CDM plano obtido de 182 dados de supernovas tomados por Riess et al. (2007). b) Probabilidade para o parâmetro de aglomeramento $\alpha$. c) Probabilidade para o parâmetro de densidade da matéria $\Omega_{M}$.

quando comparamos os vínculos obtidos supondo $H_{0}$ fixo. $\mathrm{O}$ alto valor obtido de $\Omega_{M}$ no melhor ajuste evidencia o fato de que dados em altos redshifts não vinculam bem a energia escura, o que faz com que nesse caso um modelo composto só por matéria explique bem os dados.
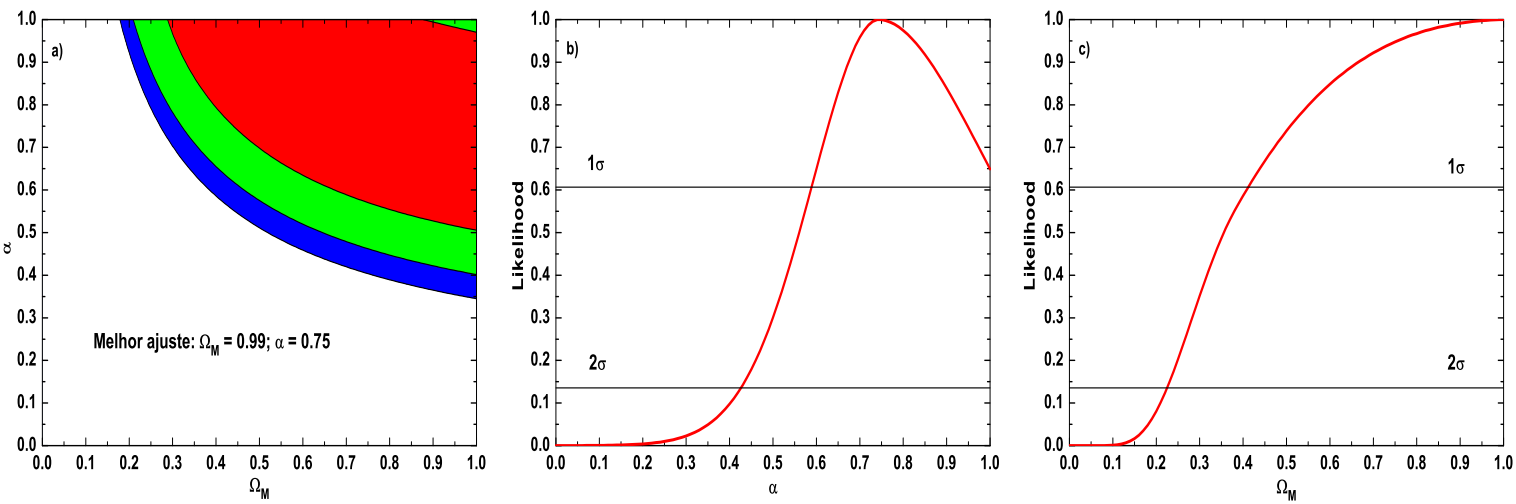

Figura B.8: a) Plano $\Omega_{M}-\alpha$ para o modelo $\Lambda$ CDM plano obtido de 69 GRBs utilizando a calibração de Schaefer. Os contornos representam níveis de significância estatística de $68.3 \%, 90 \%$ e 95.4\%, respectivamente. b) Likelihood para o parâmetro de aglomeramento $\alpha$. Obtivemos dentro de um nível de significância de $95.4 \%$ o intervalo $0.43 \leq \alpha \leq 1$. c) Likelihood para o parâmetro de densidade da matéria $\Omega_{M}$. Para esse parâmetro obtivemos dentro de um nível de significância de $95.4 \%$ o intervalo $0.23 \leq \Omega_{M} \leq 1$.

Também consideramos a amostra de 69 gamma-ray bursts calibrados para o modelo cardassiano (Mosquera Cuesta et al., 2008). Uma análise com tal amostra foi feita a fim de vincular os parâmetros $\alpha$ e $\Omega_{M}$. Chegamos aos resultados pela efetuação do teste $\chi^{2}$, que estão apresentados na figura B.9. Vemos que o resultado obtido é bem semelhante ao 
obtido quando usamos a calibração de Schaefer. Logo, a influência da calibração adotada é pequena quando a hipótese de um prior flat sobre $H_{0}$ é suposta.
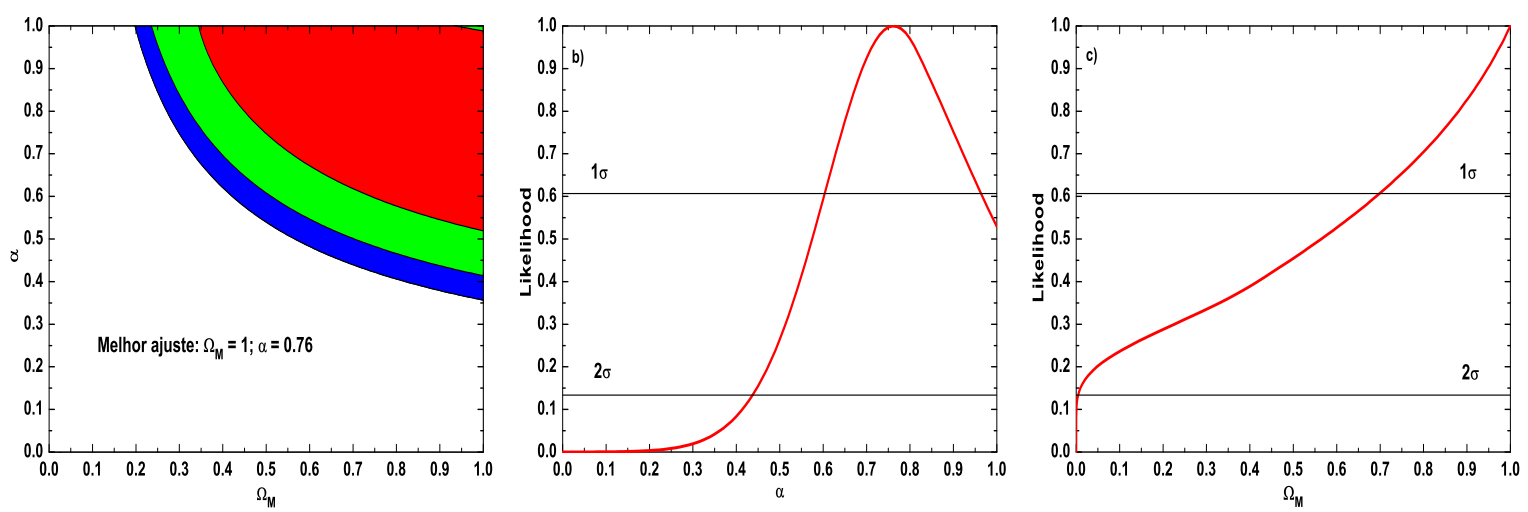

Figura B.9: a) Contornos no plano $\Omega_{M}-\alpha$ para níveis de significância estatística de $68.3 \%, 90 \%$ e $95.4 \%$, respectivamente. Estes contornos foram obtidos para o modelo $\Lambda$ CDM plano obtido de GRBs utilizando a calibração para o modelo cardassiano. b) Likelihood para o parâmetro de aglomeramento $\alpha$, onde dentro de um nível de significância de $95.4 \%$ obtivemos o intervalo $0.44 \leq \alpha \leq 1$. c) Likelihood para o parâmetro de densidade da matéria $\Omega_{M}$. Neste caso obtivemos dentro de um nível de significância de $95.4 \%$ o intervalo $0.26 \leq \Omega_{M} \leq 1$.

\section{B.2.3 Supernovas e Gamma-Ray Bursts}

Assim como quando consideramos $H_{0}$ fixo, é de suma importância combinarmos as amostras para vincularmos os parâmetros cosmológicos. O teste $\chi^{2}$ foi realizado para a amostra de 307 supernovas de Kowalski et al. (2008) (Union Compilation Data) e 69 gamma-ray bursts calibrados por Schaefer (2007). Para a análise conjunta, temos que somar os valores de $\chi^{2}$ de cada amostra, ou seja, $\chi^{2}=\chi_{S N}^{2}+\chi_{G R B}^{2}$. Os vínculos aos parâmetros $\alpha$ e $\Omega_{M}$ são apresentados na figura B.10.

Pela análise dos resultados obtidos, concluímos que quando combinamos as amostras conseguimos obter um bom vínculo sobre ambos os parâmetros, com o melhor ajuste compatível com um universo perfeitamente homogêneo e isotrópico e com $\Omega_{M}=0.30$, de acordo com outros testes observacionais.

Uma análise envolvendo as 307 supernovas da amostra de Kowalski et al. (2008) (Union Compilation Data) e os 69 gamma-ray bursts calibrados para o modelo cardassiano (Mosquera Cuesta et al., 2008) foi também considerada. Vinculamos os parâmetros $\alpha$ e $\Omega_{M}$ através do teste $\chi^{2}$ adotando uma análise conjunta. Os resultados obtidos são mostrados 

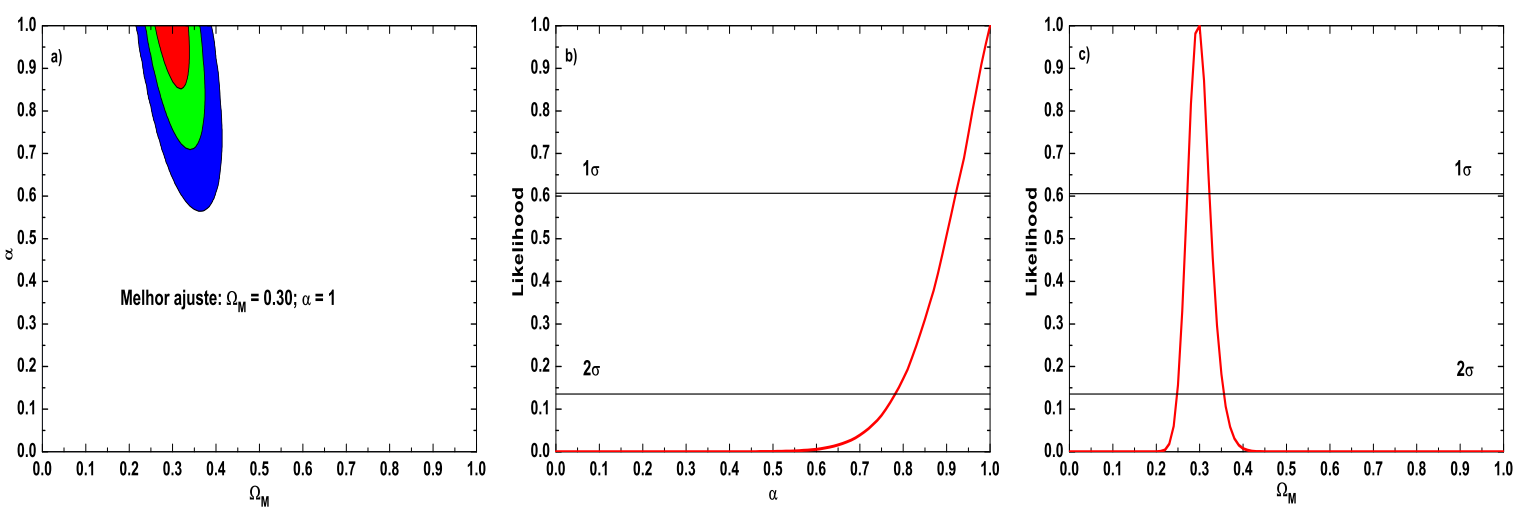

Figura B.10: a) Contornos no plano $\Omega_{M}-\alpha$ para o modelo $\Lambda$ CDM plano obtido de 307 dados de supernovas e 69 GRBs utilizando a calibração para de Schaefer, que representam níveis de significância estatística de $68.3 \%, 95.4 \%$ e $99.7 \%$, respectivamente. b) Likelihood para o parâmetro de aglomeramento $\alpha$, onde obtivemos dentro de um nível de significância de $95.4 \%$ o intervalo $0.79 \leq \alpha \leq 1$. c) Likelihood para o parâmetro de densidade da matéria $\Omega_{M}$. Obtivemos dentro de um nível de significância de $95.4 \%$ o intervalo $0.25 \leq \Omega_{M} \leq 0.35$.

na figura B.11. Os vínculos são bastante semelhantes se comparados aos obtidos com a calibração de Schaefer, o que nos faz concluir que a análise adotada é fracamente dependente da calibração utilizada.

Vemos também que a combinação das amostras mostra-se como uma ferramenta interessante para a quebra de degenerescência entre os parâmetros, fazendo com que os vínculos obtidos sejam consideravelmente melhores comparados aos testes com cada amostra. Tal fato evidencia a complementaridade dos GRBs a outros testes observacionais.

Na tabela B.2 sintetizamos os vínculos obtidos quando consideramos um prior flat sobre $H_{0}$. Verificamos que as calibrações de Schaefer e para o modelo cardassiano propiciaram vínculos semelhantes, uma indicação de que quando consideramos um prior flat sobre $H_{0}$ os resultados são fracamente dependentes da calibração. O mesmo ocorreu para a análise conjunta. Da mesma forma quando consideramos um $H_{0}$ fixo, a análise conjunta permitiu que a degenerescência entre os parâmetros $\alpha$ e $\Omega_{M}$ fosse quebrada, permitindo que ambos parâmetros fossem bem vinculados. 

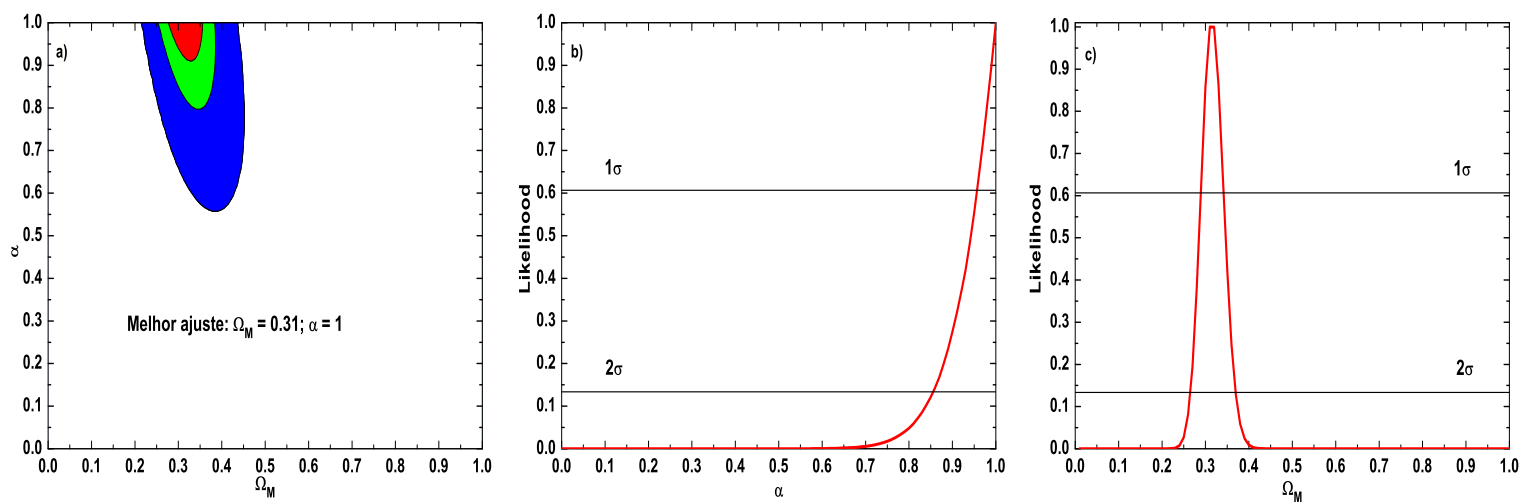

Figura B.11: a) Plano $\Omega_{M}-\alpha$ para o modelo $\Lambda$ CDM plano obtido de 307 dados de supernovas e 69 GRBs utilizando a calibração para o modelo cardassiano, onde os contornos expressam os níveis de significância estatística de $68.3 \%, 95.4 \%$ e $99.7 \%$, respectivamente. b) Likelihood para o parâmetro de aglomeramento $\alpha$. Para esse parâmetro obtivemos dentro de um nível de significância de $95.4 \%$ o intervalo $0.86 \leq \alpha \leq 1$. c) Likelihood para o parâmetro de densidade da matéria $\Omega_{M}$, onde obtivemos dentro de um nível de significância de $95.4 \%$ o intervalo $0.27 \leq \Omega_{M} \leq 0.36$.

Tabela B.2 - Vínculos para os parâmetros $\alpha$ e $\Omega_{M}$ considerando um prior flat em $H_{0} \cdot \chi_{\min }^{2}$ representa o $\chi^{2}$ mínimo obtido, o subscrito $M A$ representa o melhor ajuste obtido e $2 \sigma$ o intervalo de confiança estatística de 95.4\%. (S) representa os GRBs calibrados por Schaefer (2007) e (C) os GRBs calibrados para o modelo cardassiano (Mosquera Cuesta et al., 2008).

\begin{tabular}{lccccc}
\hline \hline \multicolumn{1}{c}{ Amostra } & $\chi_{\text {min }}^{2}$ & $\alpha_{M A}$ & $\left(\Omega_{M}\right)_{M A}$ & $2 \sigma$ & $2 \sigma$ \\
\hline SN & 311.96 & 1 & 0.29 & $0.36 \leq \alpha \leq 1.0$ & $0.24 \leq \Omega_{M} \leq 0.37$ \\
GRB(S) & 68.91 & 0.75 & 0.99 & $0.43 \leq \alpha \leq 1.0$ & $0.23 \leq \Omega_{M} \leq 1.0$ \\
GRB(C) & 68.80 & 0.76 & 1 & $0.44 \leq \alpha \leq 1.0$ & $0.26 \leq \Omega_{M} \leq 1.0$ \\
SN e GRB(S) & 390.42 & 1 & 0.30 & $0.79 \leq \alpha \leq 1.0$ & $0.25 \leq \Omega_{M} \leq 0.35$ \\
SN e GRB(C) & 394.93 & 1 & 0.31 & $0.86 \leq \alpha \leq 1.0$ & $0.27 \leq \Omega_{M} \leq 0.36$ \\
& & & & &
\end{tabular}

Nossos resultados indicam que o prior utilizado deve conter de forma satisfatória nosso conhecimento prévio do valor da grandeza. Priors muito restritivos ou que não indiquem um intervalo influem nas conclusões obtidas. Por isso, no corpo da dissertação utilizamos um prior gaussiano, uma vez que tal prior reflete melhor nosso conhecimento sobre o valor de $H_{0}$. Além disso, os vínculos obtidos também são pouco dependentes da calibração adotada, o que corrobora a consistência da análise. 